WORKING PAPER № 2005 - 35

Are the antiglobalists right? Gains-from-trade

without a walrasian auctioneer

Hector Calvo Pardo

JEL Codes : E32, D84, C62, F15

Keywords : Open economy, rational expectations, coordination, common knowledge. 


\title{
Are the Antiglobalists Right? Gains-from-Trade without a Walrasian Auctioneer
}

\author{
H. Calvo Pardo* \\ PSE (CNRS-EHESS-ENPC-ENS) and University of Southampton ${ }^{\dagger}$
}

October 30, 2005

\begin{abstract}
We examine whether the 'fear' of globalisation can be rationalised by economic theory. To do so, we depart from the standard AD/AS (partial) equilibrium model where the coordinational role of the Auctioneer is substituted by an implementation device based on learning (Guesnerie, [11]). By endowing producers with a learning ability to forecast market prices, individual profit-maximizing production decisions become interdependent in a strategic sense (strategic substitutes). Performing basic comparative statics exercises, we show that 'competitiveness' matters in a precise sense: as foreign producers gain access to the home market, home producers' ability to forecast market prices is undermined, so being their ability to forecast the profit consequences of their production decisions. When performing a standard open economy exercise in such a framework, we show that the existence of standard efficiency gains -due to the increase in competition (or spatial price stabilization)- is traded-off against coordination upon the welfare enhancing free-trade equilibrium (stabilizing price expectations). Therefore, we identify a new rationale for an exogenous price intervention in open economy targeting coordination, to allow trading countries to fully reap the benefits from trade. We illustrate this point showing that classical measures evaluating ex-ante the desirability of economic integration (net welfare gains) do not always advice integration between two expectationally stable economies.
\end{abstract}

Keywords: Open Economy, Rational Expectations, Coordination, Common Knowledge.

JELs: E32, D84, C62, F15.

\footnotetext{
${ }^{*}$ I wish to thank my PhD advisor, Prof. R. Guesnerie, for getting me started on this project. Suggestions and comments by T. Verdier have been extremely helpful. Comments by Facundo Albornoz, Pol Antràs, Gregory Corcos and Maurice Kugler are gratefully acknowledged. Seminar audiences at the U. of Alicante, U. Autonoma de Barcelona, ETSG 2005 (Dublin), FGV-EPGE (RJ), LACEA (2005) and T2M 2005 are acknowledged. Financial support from the Bank of Spain is sincerely acknowledged.

${ }^{\dagger}$ Postal address: PSE, Paris-Jourdan Sciences Economiques (Joint Research Unit CNRSEHESS-ENPC-ENS), 48 Bd Jourdan, bat. A, 75014 Paris, France. E-mail: calvo@pse.ens.fr
} 


\section{Introduction}

It is well established that one of the potential benefits of economic integration, and economic openness in general, stems in equalizing the prices of those commodities produced at different (real) costs across regions. In a standard partial equilibrium competitive framework with heterogeneous producers facing an identical demand across regions, free trade will result in lower aggregate profits for the country producing at a higher relative cost. The consumers of that country will benefit from a lower price, relative to autarky ${ }^{1}$. With symmetric cost structures across regions, differences in consumers' valuations also explain the existence of trade induced efficiency gains in real cost terms, i.e. in costs per unit of purchasing power. Overall, the increase in competition between heterogeneous producers that free trade brings in, rewards the relatively more efficient ones in (real) cost terms, as well as the higher valuation consumers, and results in a free trade equilibrium price that 'averages' the autarky prices. Hence, the meaning of (spatial) price stabilization in the abstract.

As for the abstract's wording 'stability of price expectations', we depart from a class of models where infinitesimal producers understand that the profit consequences of their individual production decisions depend on an aggregate of the decisions taken by the rest, confining them to a strategic framework where they need to form expectations on others' actions (and expectations) ${ }^{2}$. There, producers try to forecast the consequences of that aggregate on the prevailing market price, recognizing that, ultimately, the aggregate depends on the price forecasts (or expectations) that each other producer individually forms. Then, the 'stability of price expectations' refers to those situations where producers can individually forecast the actual market price, and are therefore able to individually coordinate on a course of action which confirms the forecast (expectational coordination).

Finally, the abstract's trade-off relates to the effect of free trade on the ability of producers to forecast the equilibrium price ${ }^{3}$. In the class of models considered, Guesnerie [14] convincingly argues that although strategic substituabilities

\footnotetext{
${ }^{1} \mathrm{~A}$ recent strand of the trade literature examines intra-industry effects of international trade in a general equilibrium setup with firm-heterogeneity (see Bernard et al. [2] or Melitz $[20]$ ). There, trade induces a labor reallocation from least to most productive plants, inducing endogenous entry and exit from the industry relative to autarky. Although in the simple partial equilibrium model considered we cannot consider this issue, we still capture the cross-country profit redistribution channel due to the increase in competition, absent in these models.

${ }^{2}$ This basic framework encompasses the reduced form of standard macroeconomic models in their non-noisy versions, like the Lucas aggregate supply model or a simple version of the Cagan inflation model. See Evans and Honkapohja [8] for additional details. As well, it can be seen as the competitive limit model of a large Cournot game, where producers are 'small' with respect to the market size. See Guesnerie's [11] F.N. 2, Novshek [23] or Vives [26] for further details.

${ }^{3}$ Examples of the literature on firm dynamics with heterogeneous firms, such as Hopenhayn [17] or Melitz [20], assume that firms are uncertain about their productivity and face sunk entry costs, so that they have to take forward looking decisions anticipating future probabilities of exit. Both assume that firms correctly anticipate the stationary equilibrium productivity probability distribution. In this work we study the conditions under which producers can actually learn the equilibrium probability distribution.
} 
(or complementarities) determine the sign of agents' reactions to expectations, what is instrumental for expectational coordination is the magnitude of these reactions. Spatial price equalization (or stabilization) creates a redistributional conflict between the producers of the integrating regions, leading some to expect higher prices and others lower ones, relative to autarky. These conflicting policy-induced expectations, when coupled with producers' integration-related increased heterogeneity, are likely to undermine the reliability of producers' forecasts. In consequence, spurious price volatility and multiple equilibria may arise, making more compelling an 'exogenous price intervention' after integration than it was in autarky. Then, because producers's (real) costs differ spatially, both standard efficiency gains justify a free trade policy change, and the plausibility of the welfare enhancing free-trade equilibrium price is undermined, qualifying the free trade policy change.

This new 'rationale' for intervention was first illustrated by Guesnerie's [11] one-dimensional version of Muth's [22] model where producers, endowed with more realistic abilities, managed in some cases to coordinate on the unique equilibrium, in the absence of any explicit coordinating institution ${ }^{4}$. When coordination was unsuccessful, an exogenous device could be implemented to achieve (expectational) coordination ${ }^{5}$. That insight proved general enough so as to encompass many of the standard macroeconomic models, consolidating as an alternative approach to the literature justifying the implementation of a Rational Expectations Equilibrium (REE, from here on) through (adaptive) learning. This alternative approach is called 'eductive' learning ${ }^{6}$. In this work we take advantage of this approach to relate open economy expectational stability conditions to autarkic ones. Essentially, we compare equilibria ${ }^{7}$ before and after a policy change (economic integration), which is also of interest because, as Evans and Honkapohja state, "learning is the adjustment mechanism whereby the economy is steered to the new equilibrium after a structural change" ([8], p.81).

\section{Summary of Results}

'Eductive' expectational coordination is characterized by a condition on elasticities, identified by Guesnerie [11]. This condition has a natural interpretation in open economy. When new markets are available to the producers of a particular region ceteris paribus, Guesnerie's [11] discussion on the 'stabilizing' role of a high demand elasticity suggests that producers' strategic uncertainty will be alleviated, because own production decisions become less sensible to others' expectations. Therefore, as new markets render forecasts more reliable, they favour expectational coordination. However, opening the home market to for-

\footnotetext{
${ }^{4}$ Notice that there is also a sense in which the eductive viewpoint offers new hope in overcoming some of the old arguments for coordination at the international level, as it provides conditions favouring coordination in the absence of explicit coordinating institutions.

5 This 'new rationale' for intervention is carefully developed in Guesnerie [12].

${ }^{6}$ See Guesnerie [14] for an exposition of the eductive learning approach, applications to standard macroeconomic models and its relation to the adaptive approach.

${ }^{7}$ We comment below on the connection with the global comparative statics results obtained by Milgrom and Roberts [21].
} 
eign producers ceteris paribus will have the opposite effect. Producers' decisions become more interdependent in a truly strategical sense, because an increase in the relative abundance of the product, increases the sensibility of the final price to others' production decisions, undermining the reliability of home producers' forecasts relative to autarky. Economic integration, by combining both, will have an ambiguous net effect on the reliability of producers' forecasts, and thus on coordination.

Our first result states that economic integration between regions in the linear class which are expectationally stable in isolation and value the product similarly, is expectationally stable. Also for this class, economic integration between a set of unstable regions and a set of stable ones can end up being stabilizing, provided that the unstable set is not 'too unstable' and that its demand elasticity is small relative to the integrated economy's. Given the distributional conflict created by spatial price stabilization, this is a surprising result. We would expect coordination to be increasingly difficult with the degree of heterogeneity of the system. However, recalling Guesnerie's [14] insight, it is the interplay between the magnitude of the reactions (to a given price change) and their sign what matters. Our second result shows then that spatial differences in the valuation of the produced commodity or in price-varying supply and demand elasticities undermine expectational coordination. Both introduce heterogeneity in the magnitude of the responses to the redistributional conflict created by the policy change ${ }^{8}$. This is why our first result does not extend to regions in the non-linear class even if consumers value the commodity similarly. Intuitively, spatial differences in consumers' valuations make more likely a type of 'market disruption' phenomenon which, by excluding low valuation consumers, only exacerbates the producers' strategic uncertainty, rendering own forecasts more dependent on others' forecasts.

Finally and in consequence, even if an exogenous intervention was unnecessary at the autarky level, it becomes compelling because of integration. To illustrate this fact, we compare the expectational coordination criterion with a more traditional gains-from-trade criterion from an ex-ante viewpoint. In the class of one-dimensional linear models considered, the net welfare gain from integration increases with the degree of spatial heterogeneity ${ }^{9}$. For given identical demands across regions, the gains are larger the larger the spatial differences in the aggregate costs of production. But as well, the larger the last, the more spatially dispair the supply responses to a given price change will be, and the higher the probability of expectational destabilization by economic integration. The reason we adopt an ex-ante viewpoint (before effective integration takes

\footnotetext{
${ }^{8}$ We will show below that expectational coordination is governed by a condition on first derivatives. Then, the magnitude of the reactions to the policy change is measured by changes in first derivatives. This force is absent in the linear class of models without spatial differences in consumers' valuations, explaining the counterintuitive result mentioned above.

${ }^{9}$ From a classical normative point of view, the partial equilibrium framework is a particular case of a general equilibrium economy for which Dixit and Norman [6] showed the existence of ex-post transfers that leave everybody better off. However, the effective implementation of these transfers, from an eductive viewpoint, remains an open question because it is likely to modify the strategic behaviour of producers.
} 
place) is that the appropriate criterion would necessitate computing producers' welfare when the set of rationalizable expectations equilibria is not a singleton, which is beyond the scope of the present work ${ }^{10}$.

The work proceeds as follows: In section 2, we describe the linear version of Guesnerie's [11] model, and his main results relevant to our work. The reader familiar with his work can directly start in section 3 , where we study the effect of economic integration between regions in the linear class, with and without spatial differences in the maximal willingness to pay. In section 4 , we extend the results of section 3 to integrating regions in the non-linear class. In section 5 , we compare the expectational coordination criterion to a more traditional one, which evaluates in welfare terms economic integration from an ex-ante viewpoint. Finally, in section 6 we conclude.

\section{Preliminaries}

If one is to recognize that economics is not a natural science because economic agents make forecasts that influence the time path of the system, it becomes crucial to understand how do economic agents form expectations. Faced with this problem, the modern macroeconomics literature has focused on how do economic agents 'learn'. A strand of the 'learning' literature views economic agents as statisticians who use sophisticated forecasting techniques to estimate the parameters of the law of motion governing the economic system, and on the same time taking into account that the use of these techniques shapes the motion itself. Stated otherwise, available information on the evolution of the economic system is at best incomplete even to the most sophisticated economic agent $^{11}$. The question is then whether the estimated motion would (at least) asymptotically approximate the motion consistent with agents forming a rational expectation. This is called the 'adaptive approach to learning' (or evolutive learning) and has a long lasting tradition ${ }^{12}$.

A different strand of the literature upon which we hinge here, is the 'eductive approach to learning'. This second modern approach admits that agents are rational and know the whole structure of the model describing the evolution of the system. Nevertheless, agents form expectations that need not coincide: Bernheim [3] and Pearce [24] show that rationality of the players and complete information of the game being played, even when they are 'common knowledge'

\footnotetext{
${ }^{10}$ Allen, Dutta and Polemarchakis [1] address this problem in generic competitive exchange economies with countably many competitive equilibria.

${ }^{11}$ Manski [18] presents two serious reasons in support of the incomplete information workhorse assumption: empirical data captures the result of choices, and not the expectations of decision makers when confronted with choices. Second, one cannot expect to recover objective evidence on expectations because of the selection bias (logical unobservability of counterfactual outcomes). By these reasons, he supports data collection on expectations. Recent work by Evans and Honkapohja [8] along the lines of adaptive learning, solves the design of optimal monetary policies when observed data on private agents' expectations are incorporated in the policy maker's optimal monetary rule.

${ }^{12}$ Evans and Honkapohja [9] summarize this approach and its applications.
} 
(CK), do not imply the Nash equilibrium outcome but a different solution concept called a 'rationalizable equilibrium' $\left({ }^{13}\right)$. Guesnerie [11] applies the notion of rationalizability to a version of the standard Muthian model, to show that CK of rationality and of the model are not enough for them to always coordinate their expectations on the unique REE solution defined by Muth [22]. In this sense, since the definition of a REE requires expectational coordination ${ }^{14}$, the eductive approach looks for structural conditions under which isolated independent agents' subjective expectations end up coordinating upon a REE.

In this section we present Guesnerie's [11] model, its linear version and his main results relevant to our work. The equilibrium concept will be a 'Rationalizable Expectations Equilibrium', as defined in Guesnerie's [11],[14] works.

\subsection{The Model and the Equilibrium Concept}

The model describes a two-period partial-competitive equilibrium of an agricultural commodity economy. A continuum of profit maximizing risk-neutral farmers $f \in[0,1]$ with a differentiable and strictly convex cost function $C(q, f)$ must decide the quantity $q$ to be produced a period in advance on selling, given a predictable demand $D(p)$, assumed to be downward sloping $D^{\prime}(p)<0$ and resulting from the aggregation of a continuum of identical consumers indexed by $c, D(p)=\int D(p, c) d c$. The effective equilibrium price is unknown because it depends on what other farmers will decide to produce. Therefore, the supply of each producer will also depend on the probability distribution of the price, denoted $d \mu(p)\left({ }^{15}\right)$. Since farmers are risk neutral, their production decisions will only depend on the expectation of the price $E p=\int p d \mu(p)$ :

$$
S[p, d \mu(p), f]=\left(\partial_{q} C_{f}\right)^{-1}[p, d \mu(p)] \in \arg \max _{q} \int[p q-C(q, f)] d \mu(p)
$$

Putting the Lebesgue measure on $[0,1]$, aggregate supply will be given by:

$$
S[p, d \mu(p)]=\int S[p, d \mu(p), f] d f
$$

Under the above assumptions, the Rational Expectations Equilibrium (REE) price $\bar{p}$ of this model will be given by the equality of aggregate supply and aggregate demand in expectation, computed using $d \mu(\bar{p}, f)=d \mu(\bar{p}), \forall f$ (i.e. farmers form rational expectations):

$$
\bar{p}=D^{-1}(S[\bar{p}, d \mu(\bar{p})])
$$

\footnotetext{
${ }^{13}$ Tan and Werlang [25] transform a non-cooperative game into a Bayesian decision problem where the uncertainty faced by a given agent is formed by the actions, priors over actions, priors over priors over actions, etc. of the other agents. They show that common knowledge of the actual strategies to be played is only necessary for players to play Nash strategies.

${ }^{14}$ Evans [7] asserts that a REE is in the class of Nash equilibria (in actions and beliefs).

${ }^{15}$ Strictly speaking, the probability distribution should allow for subjective probabilities and therefore be written $d \mu(p, f)$. However, the only objective difference across farmers is the cost function which should not influence the individual expectation of the market price, i.e. a farmer with lower costs cannot be reasonably expected to have a more optimistic (or pessimistic) expectation on the prevailing market price.
} 
Since there is no noise, the equilibrium $\bar{p}$ is a Perfect Foresight Equilibrium (PFE). Therefore, there exists a unique REE (PFE). Following Evans' [7] assertion according to which a REE is in the class of Nash equilibria in actions and beliefs (NE), $\bar{p}$ is also the unique $\mathrm{NE}^{16}$.

Guesnerie [11], following Bernheim [3] and Pearce [24], builds upon the gametheoretic concept of 'rationalizability' to define the 'Rationalizable-Expectations Equilibria'. These are the limit of an iterative process which views the farmers' situation as a complete information normal-form game where the set of players is the set of farmers, and their strategies, the farmers' individual quantities of the crop $s_{f} \in \mathbf{S}_{f}, \forall f\left({ }^{17}\right)$. Each farmer's payoff function is then his profit function:

$$
\left\{D^{-1}\left(\int s_{f^{\prime}} d f^{\prime}\right)\right\} s_{f}-C\left(s_{f}, f\right)
$$

For each given profile of strategies of the other farmers $\left(s_{f^{\prime}}\right)_{f^{\prime} \in[0,1]}$, the best response of farmer $f$ is the function that maximizes the above expression. The concept of a 'rationalizable solution' $R$ exhausts the implications of individual rationality and common knowledge $(\mathrm{CK})$ of rationality and of the model when considered as an iterative process taking place in 'mental time' $\tau$ (in each of the farmers' heads) following which non-best response strategies are progressively eliminated $^{18}$. Where does this iterative process start? It starts at an initial restriction $(\tau=0)$ on the players' strategy sets called anchorage assumption, which is either naturally embedded on the model at stark or exogenously given ${ }^{19}$. In either case, it is also CK. This iterative process of elimination of non-best responses will lead somewhere, defined by Pearce [24] and Bernheim [3] as a rationalizable solution $R$ :

$$
R=\left(s_{f^{\prime}}\right)_{f^{\prime}} \in \prod_{f^{\prime}}\left(\cap_{\tau=0}^{\infty} \mathbf{S}\left(\tau, f^{\prime}\right)\right)
$$

Whenever the sets of best response strategies $\mathbf{S}(\tau, f)$ shrink through 'mental time' $\tau$ to a singleton, farmers instantaneously coordinate on a unique (production) strategy. Because of the one-to-one correspondence between prices

\footnotetext{
${ }^{16}$ For an explicit formulation of this assertion in the class of models under consideration, see Desgranges and Gauthier [5].

${ }^{17}$ At this stage, it is important to understand that since the supply function is a oneto-one correspondence of the expected prevailing market price, as Guesnerie [11] points out (p.1258), the strategies are also the individual price expectations. For an exposition using price expectations, see Desgranges and Gauthier [5].

${ }^{18}$ Observe that a CK assumption is absolutely rational in a strategic context: when an individual recognizes that self-interest depends on others' actions, his conjectures on their likely behaviour are essential to the effective consecution of self intentions. The conjectures are the subjective expectations that each agent forms independently of others. But if one is to form conjectures about others' behaviour, it seems natural to recognize that others form conjectures as well in the same way as one does. Then the agent must conjecture about others' actions and conjectures. This process can go several steps further, triggered by the CK behavioural assumption.

${ }^{19}$ At this stage, it is to be understood not as an exogenous intervention, but as a robustness test that any REE should pass for it to be 'implementable' through the iterative process of learning that is being described. If the REE fails to pass the test, then exogenous price restrictions (more or less severe) can be introduced by an exogenous third party, to achieve coordination. See Guesnerie [14] for further details.
} 
and quantities, that production decision will correspond to a price expectation. As market clearing is CK, that price expectation must clear the market, and therefore coincide with the actual equilibrium price. As that equilibrium price is the unique rationalizable solution, and because the Nash solution is always rationalizable, the equilibrium price must coincide with the Nash equilibrium of the normal-form game. However, when the sets of farmers' best responses do not collapse to a singleton, full coordination is not achieved. Although the Nash equilibrium will be included in, farmers equivalently consider each of the possible rationalizable strategies as an equilibrium production decision, corresponding each to an equilibrium price expectation ${ }^{20}$.

Guesnerie [11] otains structural conditions under which, without assuming that farmers held rational expectations, the Rationalizable Expectations Equilibrium of the farmers' normal-form game described above coincides with the REE (or NE). The unique Rationalizable Expectations Equilibrium is called by him a 'Strongly Rational Expectations Equilibrium' (SREE) or 'unique rationalizable expectations equilibrium'.

\subsection{The Linear Specification}

Consider the (non-noisy) linear version of the model presented above. The demand function for the crop is given by:

$$
D(p)=\left\{\begin{array}{lr}
A-B p \text { if } 0 \leq p \leq \frac{A}{B} \equiv p_{0} \\
0 \quad \text { otherwise }
\end{array}\right.
$$

and $C(q, f)=\frac{q^{2}}{2 C_{f}}, f \in[0,1]$ constitutes the farmers' cost function. Under this linear specification, the PFE price is given $b^{21}$ :

$$
\bar{p}=\frac{A}{B+C}: C \equiv \int C_{f} d f
$$

The game that farmers play has a set of rationalizable strategies given by the limit of the iterative process of elimination of non-best responses from the strategy sets of farmers that we describe. The iteration is triggered by the CK of individual rationality and of the model, since the anchorage assumption is embedded in the structure of the model: at virtual time $\tau=0$ each farmer $f$ recognizes that equilibrium prices cannot be negative nor larger than $p_{0} \equiv \frac{A}{B}$

\footnotetext{
${ }^{20}$ It is important to stress that to compute the rationalizable equilibrium, the subjective price probability distribution and the cost function of every agent as well as market clearing are CK in the model considered. The work by Desgranges and Gauthier [5] makes clear the distinction between strategic uncertainty and model uncertainty in the linear noisy one-dimensional version of Guesnerie [11] presented here: they show that whenever the CK assumption on farmers' subjective probability beliefs is violated, the success of the iterative process is compromised. Intuitively, when the subjective probability beliefs are not CK, farmers play an incomplete information game.

${ }^{21}$ It can be checked that with the encompassing definition of the demand function $D(p)=$ $\max \{A-B p, 0\}$, with $p_{0} \equiv \min D^{-1}(0)=\frac{A}{B}$, the PFE price equals $p_{0}$ when total supply is zero.
} 
since $D\left(p_{0}\right)=0$, defining the maximum willingness to pay. Therefore each farmer deletes from his strategy set any quantity of the crop $s_{f} \geq S\left(p_{0}, f\right)$ defining the set $\mathbf{S}(0, f)=\left[0, S\left(p_{0}, f\right)\right], \forall f$. At $\tau=1$ since each farmer knows that other farmers are rational as well, each farmer knows that other farmers $\forall f^{\prime} \neq f$ will play strategies in their sets $\mathbf{S}\left(0, f^{\prime}\right)$. Therefore, total supply cannot be greater than $S\left(p_{0}\right)=\int S\left(p_{0}, f^{\prime}\right) d f^{\prime}$, which from the market clearing equation being common knowledge, each farmer deduces that the equilibrium price cannot be smaller than $p_{1}=D^{-1}\left[S\left(p_{0}\right)\right]$ and proceeds to delete from his strategy set $\mathbf{S}(0, f)$ all these quantities that are smaller than $s_{f} \leq S\left(p_{1}, f\right)$. This defines the new set of strategies $\mathbf{S}(1, f)=\left[S\left(p_{1}, f\right), S\left(p_{0}, f\right)\right]$ for every farmer $f$. Now at $\tau=2$ each farmer recognizes that the other farmers $\forall f^{\prime} \neq f$ know what he knows, and therefore play also strategies in the set $\mathbf{S}\left(1, f^{\prime}\right) \ldots$ and so on. Intuitively, each step $\tau$ corresponds to a further logical deduction step progressively exhausting the implications of the CK behavioural assumption and of the initial anchorage restriction triggering it. This process leads each farmer to individually reproduce in their heads the following sequence of (expected) prices $\left(p_{\tau}\right)_{\tau=0}^{\infty}$ :

$$
\begin{aligned}
p_{1}= & D^{-1}\left[S\left(p_{0}\right)\right]=\frac{A}{B}-\frac{C}{B} p_{0} \\
p_{2}= & \frac{A}{B}-\frac{C}{B} p_{1}=\frac{A}{B}\left[1+\left(-\frac{C}{B}\right)\right]+\left(-\frac{C}{B}\right)^{2} p_{0} \\
& \cdots \\
p_{\tau}= & \frac{A}{B}-\frac{C}{B} p_{\tau-1}=\frac{A}{B}\left[\sum_{m=0}^{m=\tau-1}\left(-\frac{C}{B}\right)^{m}\right]+\left(-\frac{C}{B}\right)^{\tau} p_{0}
\end{aligned}
$$

If this sequence has a limit, from the rationalizable solution concept, it must be the Nash equilibrium of the game $\bar{p}$. We reproduce Guesnerie's [11] proposition 1 , which establishes conditions under which farmers are able to coordinate on the PFE price $\bar{p}$. Under those conditions the equilibrium is a SREE:

Proposition 1 (Guesnerie, [11]) (i) $B>C \Longleftrightarrow \bar{p}$ is an SREE. (ii) $B \leq$ $C \Longleftrightarrow \bar{p}$ is not an SREE, and the set of rationalizable-expectations price equilibria comprises the segment $\left[0, p_{0}\right]$

The conclusion of proposition 1 can be read as 'a low elasticity of aggregate supply (small $C$ ) and a high elasticity of demand (large $B$ ) favour expectational coordination from an eductive viewpoint'. Intuitively, it can be read also as 'producers' forecasts are more reliable the lower the sensibility of their decisions to others' forecasts'. Then under condition (i), the set of farmers' rationalizable strategies that are the rationalizable solution $R$ of the farmers' game is:

$$
R=\left(s_{f^{\prime}}\right)_{f^{\prime}} \in \prod_{f^{\prime}}\left(\cap_{\tau=0}^{\infty} \mathbf{S}\left(\tau, f^{\prime}\right)\right)=\prod_{f^{\prime}} \mathbf{S}\left(\infty, f^{\prime}\right)=\left(S\left(\bar{p}, f^{\prime}\right)\right)_{f^{\prime}}
$$

If however condition (ii) is satisfied, then the price sequence $\left(p_{\tau}\right)_{\tau=0}^{\infty}$ does not have a limit and the set of farmers' rationalizable strategies that are a rational- 
izable solution $R$ of the farmers' game is:

$$
R=\left(s_{f^{\prime}}\right)_{f^{\prime}} \in \prod_{f^{\prime}}\left(\cap_{\tau=0}^{\infty} \mathbf{S}\left(\tau, f^{\prime}\right)\right)=\prod_{f^{\prime}} \mathbf{S}\left(0, f^{\prime}\right)=\times_{f^{\prime}}\left[0, S\left(p_{0}, f^{\prime}\right)\right]
$$

In situations like (ii), Guesnerie [11] identifies the minimal set of conditions sufficient to achieve full coordination, calling them 'credible price restrictions' or 'exogenous price interventions', implemented by an exogenous third party.

In this particular example, the model definition embeds the initial anchorage assumption. Furthermore, it is not 'close' to the equilibrium outcome. Then, under the (i) condition, the equilibrium price is 'Globally SR'. In general, when no such natural embedding exists, the anchorage assumption is exogenously specified. When the model considered is non-linear, the anchorage assumption is settled 'close' to the REE under scrutiny and the analysis is local (because there might exist multiple equilibria, which we assume locally determinate). Then, when the iterative process converges, the equilibrium is called 'Locally SR' or 'SR with respect to the CK anchorage assumption'. When the iterative process does not converge, the 'credible price restrictions' or 'exogenous price interventions' qualify the above definitions to be 'SR with respect to these restrictions'. For non-linear versions of the economy under study, the iterative process describing farmers' eductive learning can be characterized by the second iterate of the cobweb function $\varphi(.) \equiv D^{-1}[S()],. \varphi^{2}(.) \equiv \varphi[\varphi()$.$] , conditional$ to the CK initial restriction ${ }^{22}$, denoted $V(\bar{p})$ :

Proposition 2 (Guesnerie [11]):

(i) If $\left|\varphi^{\prime}(p)\right|<1 \Leftrightarrow S^{\prime}(p)<\left|D^{\prime}[S(p)]\right|, \forall p$ and if there is a credible price restriction (floor or ceiling), then $\bar{p}$ is a SREE subject to the given price restriction.

(ii) If $\left|\varphi^{\prime}(\bar{p})\right|<1$, there is a credible price restriction (floor or ceiling) s.t. $\bar{p}$ is a SREE subject to the given price restriction.

(iii) If $\left|\varphi^{\prime}(\bar{p})\right|>1$, and if the graph of $\varphi^{2}($.$) intersects transversely the 45-$ degree line more than once, then there is a credible price restriction (floor or ceiling) s.t. $\left[p_{c 1}, p_{c 2}\right]$ is the set of rationalizable-expectations equilibrium prices subject to the given price restriction, where $p_{c 2}=\varphi\left(p_{c 1}\right), \varphi^{2}\left(p_{c t}\right)=p_{c t}, t=1,2$ define cycles of order two of the cobweb function $\left({ }^{23}\right),\left({ }^{24}\right)$.

The results in section 3 will provide examples of each of these cases.

\footnotetext{
${ }^{22}$ Subject to the condition that $\lim _{\tau \rightarrow \infty}\left(\varphi^{2}\right)^{\tau}\left(p_{0}\right)=\lim _{\tau \rightarrow \infty} \varphi^{2 \tau}\left(p_{0}\right)=\bar{p}, p_{0} \in V(\bar{p})$

${ }^{23}$ For a proof of the general statement which includes cases (ii) of proposition 1 and this case (iii), see Bernheim [3], proposition 5.2., part (a).

${ }^{24}$ This is trivially true if $\left[p_{c 1}, p_{c 2}\right] \subset V(\bar{p})$. If however $V(\bar{p}) \subseteq\left[p_{c 1}, p_{c 2}\right]$, the learning dynamics will also converge to the set $\left[p_{c 1}, p_{c 2}\right]$, but, as discussed by Guesnerie [14], the CK anchorage assumption must then be understood not as a 'hypothetical' restriction, but as resulting from a non-enforceable 'exogenous price intervention'.
} 


\section{Integration of Linear Economies}

Most of the international trade literature concerns comparative statics excercises on the effect of changes in the production structure (factor endowments or production techniques) on the equilibrium outcome operated via the mobility of commodities or factors. The consequences on factors and commodity prices are corollaries of the comparative statics exercise under the same or alternative restrictions. However, they all necessitate at least two commodities for the exchange channel to operate. In the class of agricultural economies considered, there is only a single homogenoeus crop produced at different costs depending on farmers' technologies. From the expectational stability viewpoint, the open economy device introduces heterogeneity in the autarkic economy, which according to Guesnerie's [14] general intuition (GI2), should undermine its expectational stability. A related way to understand the exercise is to assume that non-increasing returns to scale producers play a large oligopoly game with strategic substituabilities, the equilibrium of which is globally perturbed by the integration policy. The question would then be whether the dominance solvability of the autarkic equilibrium is robust to the introduction of heterogeneity (amenable to an open economy device) ${ }^{25}$.

Although the answer will be related to the factors favouring coordination upon the integrating regions autarkic equilibrium (propositions 1,2 above), the answer is not immediate. From the comparative statics excercises of partial equilibrium, we know that aggregating demand curves results in a more elastic demand curve. According to proposition 1, a higher demand elasticity favours expectational coordination. However, and by the same reason, aggregation of supply curves is detrimental to eductive coordination. As economic integration entails both, it does not necessarily undermine the coordinational ability of farmers. Actually, mere replication of the Home economy will not affect its degree of expectational stability.

To see it, consider the linear class of agricultural economies indexed by $n \in \mathbf{N}=\{1 \ldots N\}$ characterized by a set of risk neutral farmers $f_{n} \in[0,1]$ living in region $n$ with strictly convex cost structures $C\left(s_{f_{n}}, f_{n}, n\right)=\frac{\left(s_{f_{n}}\right)^{2}}{2 C_{f_{n}}(n)}$ facing a (weakly) decreasing demand function $D_{n}(p) \equiv \int D_{n}\left(p, c_{n}\right) d c_{n}=\max \left\{A_{n}-B_{n} p, 0\right\}$ arising from a continuum of individual consumers living in that region ${ }^{26}$. Suppose that the $N$ economies in the linear class are identical and decide to integrate (fix $n=n_{0}, \forall n$ and call economy $n_{0}$ the Home economy). The aggregate supply of such a global agricultural economy will be given by the sum of the aggregate supply functions of each of the $N$ regions, $S(p)=\sum_{n=1}^{N} S_{n}(p)=$ $N \int C_{f_{n_{0}}}\left(n_{0}\right) p d f_{n_{0}}=N S_{n_{0}}(p)$. So will the aggregate demand: $D(p)=\sum_{n=1}^{N} D_{n}(p)=$ $N D_{n_{0}}(p)$. Substituting these definitions in proposition 1 above, we can immediately observe that the PFE-price is given by:

$$
\bar{p}=\bar{p}_{n_{0}}=\frac{A_{n_{0}}}{B_{n_{0}}+C_{n_{0}}}
$$

\footnotetext{
${ }^{25}$ See Vives [26] ch.4.4. for a synthetic presentation of large Cournot markets.

${ }^{26}$ Throughout we assume that $A_{n}, B_{n}>0, \forall n \in \mathbf{N}$. Notice that $p_{0} \equiv \min D_{n}^{-1}(0)=\frac{A_{n}}{B_{n}}$.
} 
Then, the conditions under which farmers will be able to individually predict the PFE-price $\bar{p}$ coincide with those of proposition 1:

Proposition 3 (i) $B_{n_{0}}>C_{n_{0}} \Longleftrightarrow \bar{p}$ is an SREE. (ii) $B_{n_{0}} \leq C_{n_{0}} \Longleftrightarrow \bar{p}$ is not an SREE, and the set of rationalizable-expectations price equilibria comprises the segment $\left[0, p_{0}\right]$.

A perhaps more interesting result is that this proposition extends to the integration of $N$ identical non-linear agricultural economies ${ }^{27}$. However, when considered in isolation, the effect of increasing the number of farmers facing a given aggregate demand curve is detrimental to the eductive stability of the equilibrium $^{28}$. Consider our Home economy $n=n_{0}$. Suppose that in addition to the Home farmers, those from the rest of the regions $\mathbf{N} \backslash\left\{n_{0}\right\}$ can also sell in the Home crop market. Denote by $C_{\Sigma}=C_{n_{0}}+\sum_{n \neq n_{0}} C_{n}$ the aggregate cost parameter characterizing the total supply of the crop. The PFE price is $\bar{p}=\frac{A_{n_{0}}}{B_{n_{0}}+C_{\Sigma}}$, which when:

Proposition 4 (i) $B_{n_{0}}>C_{\Sigma} \Longleftrightarrow \bar{p}$ is an SREE. (ii) $B_{n_{0}} \leq C_{\Sigma} \Longleftrightarrow \bar{p}$ is not an SREE, and the set of rationalizable-expectations price equilibria comprises the segment $\left[0, p_{0}\right]$. (iii) Increasing the number of farmers is detrimental to expectational stability.

Proof. Compute the limit $\lim _{\tau \rightarrow+\infty} p_{\tau}$ of the price sequence:

$$
p_{\tau}=\frac{A_{n_{0}}}{B_{n_{0}}}\left[\frac{1-\left(-\frac{C_{\Sigma}}{B_{n_{0}}}\right)^{\tau}}{1-\left(-\frac{C_{\Sigma}}{B_{n_{0}}}\right)}\right]+\left(-\frac{C_{\Sigma}}{B_{n_{0}}}\right)^{\tau} p_{0}
$$

Part (iii) follows trivially from the definition of $C_{\Sigma}$, (i) and noting that replicating the supply side of the Home economy makes $C_{\Sigma}=N C_{n_{0}}$.

Part (iii) states that the set of rationalizable solutions of the Home economy $R_{n_{0}}$ will strictly include the set of rationalizable solutions of the global agricultural economy $R$ of proposition 3: $R_{n_{0}} \supset R$. As the aggregation of supply curves increases the elasticity of the resulting aggregate supply schedule, each farmer's quantity choice becomes more sensible to other farmers' choices, rendering their predictions of the market clearing price less accurate. Intuitively, as new entrants gain access to the Home market, the relative scarcity of the home produced commodity decreases, intensifying competition and lowering the price and profits of home producers, compelling their forecasts to increasingly rely on the strategic component of what do others expect, so undermining expectational coordination $^{29}$. Therefore, opening the Home market to Foreign competitors is

\footnotetext{
${ }^{27}$ See the next section.

${ }^{28}$ As Vives [26] discusses for large Cournot games, the effect parallels adverse impact on dominance solvability of the equilibrium from increasing the number of producers without replicating the demand.

${ }^{29}$ Notice that the proposition is silent about the relative efficiency of the new foreign entrants: actually they can even produce at higher real costs (in terms of Home purchasing power, as we abstract from aggregate demand changes). Therefore, it is related to a pure scarcity effect relative to autarky, that exists because entry is exogenous.
} 
destabilizing, in the precise sense of producers' undermined ability to forecast the market clearing price ${ }^{30}$.

Replication of the Home demand without replicating the supply side shows the beneficial role of the demand elasticity on the expectational stability of the resulting PFE price, given now by $\bar{p}=\frac{N A_{n_{0}}}{N B_{n_{0}}+C_{n_{0}}}$. Then when:

Proposition 5 (i) $N B_{n_{0}}>C_{n_{0}} \Longleftrightarrow \bar{p}$ is an SREE. (ii) $N B_{n_{0}} \leq C_{n_{0}} \Longleftrightarrow$ $\bar{p}$ is not an SREE, and the set of rationalizable-expectations price equilibria comprises the segment $\left[0, p_{0}\right]$. (iii) Increasing the number of consumers favours stability.

Proof. For parts (i),(ii) compute the limit $\lim _{\tau \rightarrow+\infty} p_{\tau}$ of the price sequence in the previous proposition after replacing $\left(-\frac{C_{\Sigma}}{B_{n_{0}}}\right)$ by $\left(-\frac{C_{n_{0}}}{N B_{n_{0}}}\right)$. Part (iii) follows from (i) and $N B_{n_{0}}>B_{n_{0}}$.

Intuitively, part (iii) states that as the number of consumers increases, the demand becomes more sensible to price changes because the relative scarcity of the home produced commodity increases. Then, higher prices and profits are expected relative to autarky, relaxing home competition and reducing the weight of the strategic component in producers' forecasts (forecasting others' forecasts), which favours expectational coordination. Therefore, opening new markets for the Home producers is stabilizing, in the precise sense that producers' expectations become more reliable ${ }^{31}$.

Notice that this proposition is not the exact analogue of proposition 4 . However, defining $D(p)=\max \left\{\sum_{n}\left(A_{n}-B_{n} p\right), 0\right\} \equiv \max \left\{A_{\Sigma}-B_{\Sigma} p, 0\right\}$ and imposing the additional condition $\frac{A_{n}}{B_{n}}=\frac{A_{n^{\prime}}}{B_{n^{\prime}}}, \forall n, n^{\prime} \in \mathbf{N}$ the exact analogue obtains. This additional condition imposes the equality of the maximal willingnesses to pay for the crop across regions, i.e. some 'homogeneization' of consumers' valuation of the produced commodity. Its role on the expectational stability of the equilibrium price is the subject of the next two subsections.

\subsection{From Global to Local Stability Conditions}

In the class of linear economies considered, the anchorage assumption is embedded in the model and it is unnecessary to specify it exogenously. Furthermore, the autarkic expectational stability test of the PFE price is 'global' in the sense that the anchorage assumption is not 'close' to the equilibrium. The same is true for the PFE price of the integrated economy, provided that the consumers of different regions value the crop 'similarly', i.e. provided that consumers' maximal willingness to pay is identical across regions: $\frac{A_{n}}{B_{n}}=\frac{A_{n^{\prime}}}{B_{n^{\prime}}}, \forall n, n^{\prime} \in \mathbf{N}$.

\footnotetext{
${ }^{30}$ However, this proposition does not generalize to general non-linear schedules. In the next section we provide an example where increasing the number of producers stabilizes expectations.

${ }^{31}$ This proposition does neither extend to general non-linear schedules. In the next section we give an example where increasing the number of consumers destabilizes expectations.
} 
With the same notation as previously, we define the regional integration demand and supply by $D(p)=\max \left\{A_{\Sigma}-B_{\Sigma} p, 0\right\}, S(p)=C_{\Sigma} p$. The PFE price of the regional integration of $N$ economies in the linear class is:

$$
D(\bar{p})=S(\bar{p}) \Longleftrightarrow \bar{p}=\frac{A_{\Sigma}}{B_{\Sigma}+C_{\Sigma}}
$$

and will be expectationally stable when:

Proposition 6 Suppose that $\frac{A_{n}}{B_{n}}=\frac{A_{n^{\prime}}}{B_{n^{\prime}}}, \forall n, n^{\prime} \in \mathbf{N}$. Then: (i) $B_{\Sigma}>C_{\Sigma} \Longleftrightarrow \bar{p}$ is an SREE. (ii) $B_{\Sigma} \leq C_{\Sigma} \Longleftrightarrow \bar{p}$ is not an SREE, and the set of rationalizableexpectations price equilibria comprises the segment $\left[0, p_{0}\right]: p_{0}=\frac{A_{\Sigma}}{B_{\Sigma}}$. (iii) The regional integration of $N$ autarkically expectationally stable economies is expectationally stable, but the converse is false.

Proof. See appendix 0.

Intuitively, part (iii) states that it is not regional integration per se what undermines expectational coordination, but the integration with expectationally unstable regions. And even then, if the set of stable economies is sufficiently stable, economic integration can favour expectational coordination. This is a surprising conclusion in light of Guesnerie's [14] general intuition (GI2), which states that heterogeneity is detrimental to expectational coordination ${ }^{32}$.

Actually, if we remove the condition imposing equal maximal willingnesses to pay for the crop across regions, the regional integration demand becomes non-linear (piece-wise linear) and the results of propositon 1 above do not apply anymore. We have to resort to a local analysis of expectational stability, but as in this model the anchorage assumption is naturally embedded in the definition and not necessarily 'close' to the PFE, we are in the class of situations described by Guesnerie [14], case I.2.(i).

To exemplify it, suppose that we applied the 'expectational stability test' of the above proposition $\left(\frac{C_{\Sigma}}{B_{\Sigma}}<1\right)$ to the regional integration of two economies $n=\{1,2\}$ in the linear class $\mathbf{N}$, such that $\frac{A_{2}}{B_{2}} \geq \frac{A_{1}}{B_{1}}$. Two kinds of misleading conclusions are likely to emerge, respectively depicted in figures 1 and 2 below:
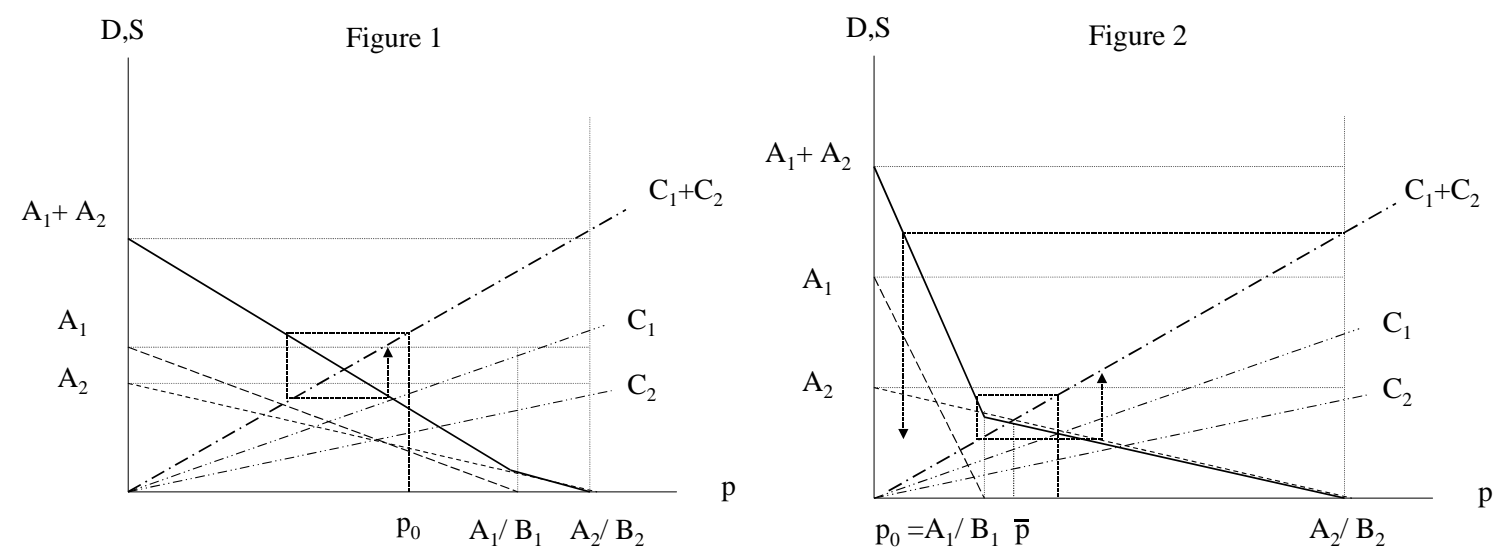

${ }^{32}$ The next section qualifies this conclusion. 
In figure 1 the (global) 'expectational stability test' fails and nevertheless the PFE price is (locally) expectationally stable. Notice that in addition, the two regions are autarkically expectationally stable. In figure 2 , the (global) 'expectational stability test' is passed, although the PFE price is (locally) expectationally unstable. What is even more striking is that both regions are expectationally stable in autarky ${ }^{33}$. In the next subsection we fully develop a two-region example and extend proposition 6 to the case where condition $\frac{A_{n}}{B_{n}}=\frac{A_{n^{\prime}}}{B_{n^{\prime}}}, \forall n, n^{\prime} \in \mathbf{N}$ does not hold.

\subsection{Structural Heterogeneity}

In this subsection we study the eductive stability of the PFE price when differences in the maximal willingnesses to pay across regions in the linear class are allowed for ${ }^{34}$. These differences render piece-wise linear the cobweb characterization of the eductive learning process (with respect to the autarkic cobweb function, which is linear) with two main consequences: First, from the comparison of Guesnerie's [11] propositions 1 and 2, the necessity of an 'exogenous price intervention' is more stringent if expectational coordination is to be maintained at the global level. This is reminiscent of the traditional need to coordinate regional social planners at the open economy level to fulfil pre-trade national goals, and it can be then understood as a new 'rationale' justifying an exogenous intervention after integration ${ }^{35}$. Second, and in consequence, the study of its convergence must be local, in the sense that the CK anchorage assumption must be settled 'close' to the PFE price. The problem is that the definition of the regionally integrated model already embeds an anchorage assumption which is not 'close', leading in some cases to the type of inconsistencies adduced by Guesnerie's [14] case I.2.(i). To give a precise content to these statements, we present a simple two-region integration exercise. Then we extend proposition 6 to accomodate spatial differences in consumers' valuations.

\subsubsection{A Robust Example}

Consider the regional integration of two economies $n=\{1,2\}$ in the linear class $\mathbf{N}$, such that $\frac{A_{2}}{B_{2}} \geq \frac{A_{1}}{B_{1}}$. Accordingly, and from the definition of regional demands, $p_{0}^{n} \equiv \min D_{n}^{-1}(0)=\frac{A_{n}}{B_{n}}, n=1,2$. Keeping the same notation, after integration farmers' demand will be $D(p)=\sum_{n} D_{n}(p) \mathbf{1}_{\left\{p \leq p_{0}^{n}\right\}}$, where $\mathbf{1}_{\left\{p \leq p_{0}^{n}\right\}}$ denotes the standard indicator function, taking value 1 only if the $n$-region consumers can afford to buy the crop at price $p$, and zero otherwise ${ }^{36}$. Then, the PFE price $\bar{p}$

\footnotetext{
${ }^{33}$ But also more intuitive, in the sense that heterogeneity is detrimental to expectational coordination.

${ }^{34}$ We will assume throughout that the region with a relatively more elastic demand will have the lower maximal willigness to pay for the crop. This assumption can be dispensed with and the conclusions still hold.

${ }^{35}$ The precise instruments, or the study of their effective implementation, are left for future work.

${ }^{36}$ In this particular example, we can alternatively characterize the demand function as $D(p)=\max \left\{A_{\Sigma}-B_{\Sigma} p, A_{2}-B_{2} p, 0\right\}$.
} 
will be given by:

$$
\bar{p}=\max \left\{\frac{A_{\Sigma}}{B_{\Sigma}+C_{\Sigma}}, \frac{A_{2}}{B_{2}+C_{\Sigma}}\right\}
$$

The PFE price is represented in figure 3 below $^{37}$ as a function of the aggregate supply cost parameter $C_{\Sigma}, \bar{p}\left(C_{\Sigma}\right)$. We have parameterized the difference in the maximal willingnesses to pay by $\bar{C}_{\Sigma}=A_{2}\left[\frac{B_{1}}{A_{1}}-\frac{B_{2}}{A_{2}}\right]$. We can see that the PFE price changes for values of the aggregate supply cost parameter above and below $\bar{C}_{\Sigma}$. Values of $C_{\Sigma}$ above $\bar{C}_{\Sigma}$ indicate that both regional markets will be served after integration, whereas values below indicate that only the highest valuation region will be served ( $n=2$, given our assumptions). The case where $\bar{C}_{\Sigma}=0$ corresponds to the equality of maximal willingnesses to pay of proposition 6 -only values above $\bar{C}_{\Sigma}$ are allowed-.
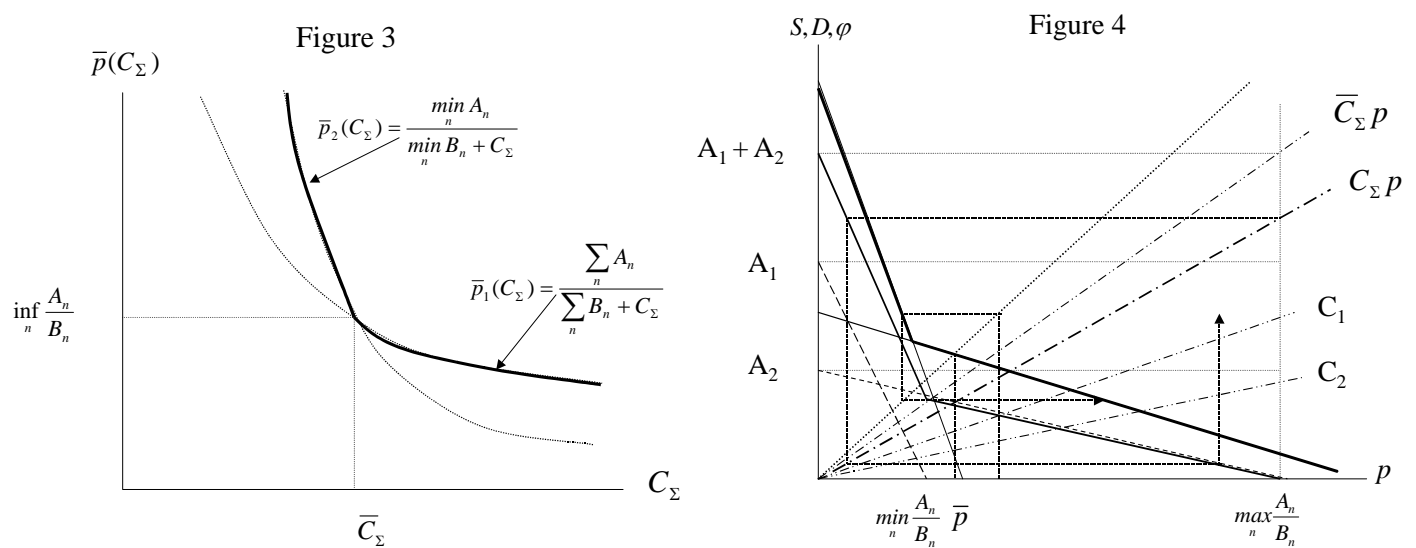

The learning dynamics of the regional integration PFE price are characterized by the piece-wise linear cobweb function $\varphi(p) \equiv D^{-1}[S(p)]$, with the following analytic form ${ }^{38}$ :

$$
\begin{aligned}
\varphi\left(p ; C_{\Sigma}\right) & =\max \left\{\varphi_{1}\left(p ; C_{\Sigma} \geq \bar{C}_{\Sigma}\right), \varphi_{2}\left(p ; C_{\Sigma} \leq \bar{C}_{\Sigma}\right)\right\} \\
& =\left\{\begin{array}{l}
\varphi_{2}\left(p ; C_{\Sigma} \leq \bar{C}_{\Sigma}\right) \text { if } p \leq p^{i} \\
\varphi_{1}\left(p ; C_{\Sigma} \geq \bar{C}_{\Sigma}\right) \text { if } p \geq p^{i}
\end{array}\right.
\end{aligned}
$$

Where $\varphi_{1}\left(p ; C_{\Sigma} \geq \bar{C}_{\Sigma}\right)=\frac{A_{\Sigma}}{B_{\Sigma}}-\frac{C_{\Sigma}}{B_{\Sigma}} p$ coincides with the linear cobweb function of characterizing the learning dynamics when the condition $\frac{A_{1}}{B_{1}}=\frac{A_{2}}{B_{2}} \Longleftrightarrow \bar{C}_{\Sigma}=0$ is satisfied, while $\varphi_{2}\left(p ; C_{\Sigma} \leq \bar{C}_{\Sigma}\right)=\frac{A_{2}}{B_{2}}-\frac{C_{\Sigma}}{B_{2}} p$ corresponds to the case in which $\frac{A_{2}}{B_{2}} \geq \frac{A_{1}}{B_{1}}$ and $C_{\Sigma} \leq \bar{C}_{\Sigma}$. Therefore, when $\frac{A_{2}}{B_{2}} \geq \frac{A_{1}}{B_{1}}$ but $C_{\Sigma} \geq \bar{C}_{\Sigma}$, the conclusions of proposition 6 apply even with different maximal valuations across

\footnotetext{
${ }^{37}$ Recall that we assumed throughout $A_{1} \geq \ldots \geq A_{N}>0$ and $B_{1} \geq \ldots \geq B_{N}>0$. Then when $n=\{1,2\}, \min A_{n}=A_{2}$ and $\min B_{n}=B_{2}$.

${ }^{38}$ In appendix 1 it is shown that $\varphi^{\prime}() \leq$.0 and that $\varphi(\bar{p})=\bar{p}$. These are general properties of the cobweb function in the class of economies under study.
} 
regions. $p^{i}$ is the price at which both functions $\varphi_{1}(),. \varphi_{2}($.$) intersect \left({ }^{39}\right)$. In figure 4 above, we have depicted the cobweb function $\varphi($.$) when \frac{A_{2}}{B_{2}} \geq \frac{A_{1}}{B_{1}}$ and $C_{\Sigma} \leq \bar{C}_{\Sigma}$ : then only region 2 consumers will be able to afford the consumption of the crop at the prevailing PFE price $\bar{p}$. Also notice that the conclusions of proposition 6 do not hold: the global 'expectational stability test' is satisfied, but the PFE price is (locally) expectationally unstable. When the economy under study is non-linear, proposition 2 above provides conclusions on the basis of the second iterate of the cobweb function $\varphi^{2}($.$) , given by { }^{40}$ :

$\varphi^{2}(p)=\left\{\begin{array}{c}\left(\varphi_{1} \circ \varphi_{2}\right)(p) \text { if } p \leq p_{\text {inf }}^{i} \\ \left(\varphi_{1} \circ \varphi_{1}\right)(p) \mathbf{1}_{\left\{p_{\text {inf }}^{i}=p^{i}\right\}}+\left(\varphi_{2} \circ \varphi_{2}\right)(p) \mathbf{1}_{\left\{p_{\text {inf }}^{i}=p^{i \prime}\right\}} \\ \left(\varphi_{2} \circ \varphi_{1}\right)(p) \text { if } p \geq p_{\text {sup }}^{i}\end{array}\right.$ if $p \in\left(p_{\text {inf }}^{i}, p_{\text {sup }}^{i}\right)$

Where $p^{i \prime}$ denotes a second intersecting price ${ }^{41}$ satisfying $\varphi_{1}\left[\varphi\left(p^{i \prime}\right)\right]=\varphi_{2}\left[\varphi\left(p^{i \prime}\right)\right]$. We define ${ }^{42} p_{\text {inf }}^{i}=\max \left\{\min \left\{p^{i \prime}, p^{i}\right\}, p_{1}^{\prime}\right\}$ and $p_{\text {sup }}^{i}=\min \left\{\max \left\{p^{i \prime}, p^{i}\right\}, p_{\infty}\right\}$, which constitute the two non-differentiability points of the piece-wise linear function $\varphi^{2}($.$) . Finally \mathbf{1}_{\left\{p_{\mathrm{inf}}^{i}=p^{i}\right\}}$ takes value 1 if $p_{\mathrm{inf}}^{i}=p^{i}$ and 0 otherwise. To gain some intuition on its shape, figure 5 depicts the second iterate of the cobweb function corresponding to the parameterization of figure 4 -the cobweb $\varphi($.$) and$ its second iterate $\varphi^{2}($.$) are superposedly depicted to facilitate the comparison-:$

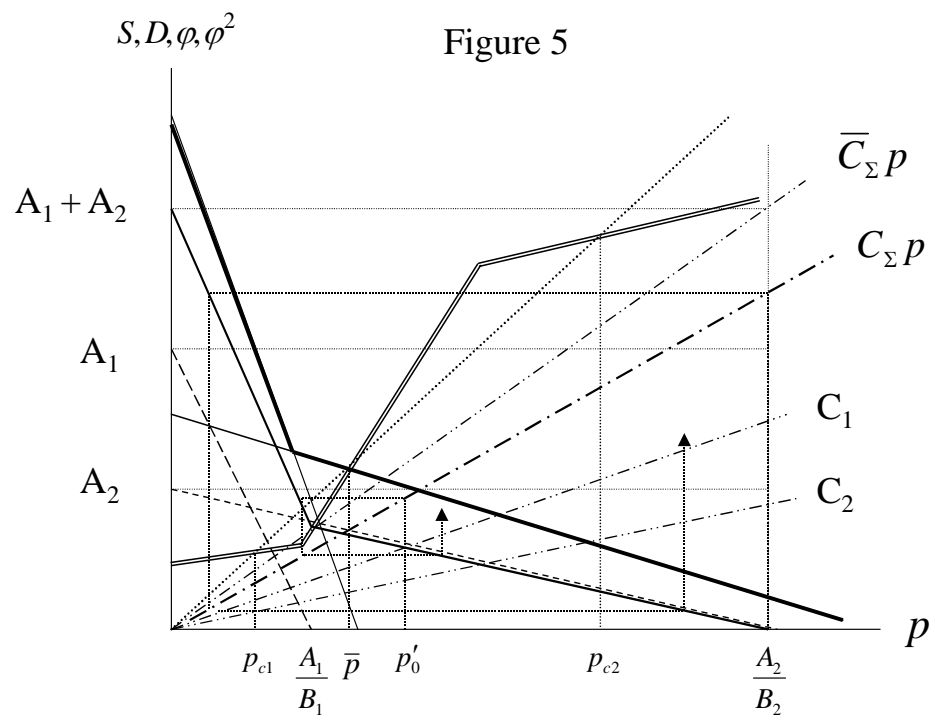

\footnotetext{
${ }^{39}$ For the derivation of the cobweb function and the expression of the intersection price, see appendix 1 .

${ }^{40}$ Notice that the learning dynamics characterized by it adopt the form of a functional piece-wise linear difference equation. See appendix 2 for the derivation, and appendix 3 for its properties.

${ }^{41}$ See appendix 2 for its derivation, explicit formulation and properties $1-5$.

${ }^{42}$ See observation 2 of appendix 2 for the definitions of $p_{1}^{\prime}, p_{\infty}$ the interest of which is merely technical.
} 
Observe that the second iterate of the cobweb function is monotonically increasing, that it satisfies $\varphi^{2}(\bar{p})=\varphi[\varphi(\bar{p})]=\varphi(\bar{p})=\bar{p}$, and that for the particular parameterization represented in figures 4 and 5 , the two non-differentiability points are given by $p^{i \prime}>p^{i}=\min _{n} \frac{A_{n}}{B_{n}}=\frac{A_{1}}{B_{1}}$. In figure 5 , the two main consequences adduced can be illustrated: First, even if both regions were expectationally stable before integration (check figure 4), the resulting PFE price is 'expectationally unstable' after integration. This is in line with the intuition that heterogeneity is detrimental to expectational coordination, qualifying the conclusions of proposition 6 above. Second, the embedded anchorage assumption $p_{0}=\max _{n} \frac{A_{n}}{B_{n}}=\frac{A_{2}}{B_{2}}$ is not 'local'. Conditional on that 'initial price restriction' $p_{0}$, the learning process converges but not to the PFE $\bar{p}$. It converges to $p_{c 2}$, belonging to the set $\left[p_{c 1}, p_{c 2}\right]$ of rationalizable-expectations equilibria, containing $\bar{p}$. If the 'local' approach had been adopted, the initial price restriction, denoted $p_{0}^{\prime}$ in the picture, would have rather been set in a neighbourhood of the PFE, $N_{\epsilon}(\bar{p})=(\bar{p}-\epsilon, \bar{p}+\epsilon)$. Then the learning dynamics depicted in figure 5 would diverge, but not forever: the process stops at $\left[p_{c 1}, p_{c 2}\right]$. This provides an illustration of Guesnerie [14], case I.2.(i). To avoid the type of inconsistency adduced, he suggests to pick $p_{0}$ outside the set of rationalizable prices $\left[p_{c 1}, p_{c 2}\right]$, but 'close' to it.

Then, the most salient result is:

Proposition 7 Set $\mathbf{N}=\{1,2\}$. If $C_{\Sigma} \geq \bar{C}_{\Sigma}$ the results of proposition 6 extend to the case where $\exists n, n^{\prime} \in \mathbf{N}: \frac{A_{n}}{B_{n}} \neq \frac{A_{n^{\prime}}}{B_{n^{\prime}}}$. If however $C_{\Sigma}<\bar{C}_{\Sigma}$ then even if both economies were autarkically expectationally stable, the global equilibrium price can end up being unstable.

Proof. (See the results in Table A4.1 in appendix 4 and the corresponding proofs)

Intuitively, a large disparity in consumers' regional valuations renders farmers' forecasts increasingly unreliable because it renders a 'market disruption' phenomenon more likely: If as a result of regional integration the PFE price is 'too high', the consumers from the low-valuation region will be excluded ('market disruption') with the adverse net effect of a pure increase in the number of farmers' competitors, studied in proposition 4.

The next proposition generalizes this result to the regional integration of $N$ economies in the linear class, such that $\exists n, n^{\prime} \in \mathbf{N}: \frac{A_{n}}{B_{n}} \neq \frac{A_{n^{\prime}}}{B_{n^{\prime}}}$. From the discussion of the previous example, we adopt a 'local' approach of convergence of the learning dynamics. Proposition 2 characterizes the local eductive stability condition, which can be rewritten as:

$$
\begin{aligned}
\varphi^{\prime}(\bar{p}) & =\varkappa_{\left\{n: \bar{p} \leq p_{0}^{n}\right\}}\left[\sum_{n} \frac{D_{n}^{\prime}(\bar{p})}{\sum_{n} D_{n}^{\prime}(\bar{p})} \varphi_{n}^{\prime}(\bar{p})\right] \\
& =\varkappa_{\left\{n: \bar{p} \leq p_{0}^{n}\right\}}\left[\sum_{n} \alpha_{n} \varphi_{n}^{\prime}\left(\bar{p}_{n}\right)\right]
\end{aligned}
$$


The second equality follows from linearity, $\varphi_{n}^{\prime}(\bar{p})=\varphi_{n}^{\prime}\left(\bar{p}_{n}\right), \forall n$. The factor $\varkappa_{\left\{n: \bar{p} \leq p_{0}^{n}\right\}}$ captures the possibility of spatial differences in the maximal willingnesses to pay, and is defined as:

$$
\varkappa_{\left\{n: \bar{p} \leq p_{0}^{n}\right\}} \equiv \frac{\sum_{n} D_{n}^{\prime}(\bar{p})}{\sum_{n: \bar{p} \leq p_{0}^{n}} D_{n}^{\prime}(\bar{p})} \geq 1
$$

with $\sum_{n: \bar{p} \leq p_{0}^{n}} D_{n}^{\prime}(\bar{p})=\sum_{n} D_{n}^{\prime}(\bar{p}) \mathbf{1}_{\left\{\bar{p} \leq p_{0}^{n}\right\}}$, from taking the price derivative of the regionally integrated demand function. The denominator sums the regional demand elasticities at the open economy equilibrium price, whenever the quantities demanded are positive. The numerator sums the regional demand elasticities irrespectively of whether open economy equilibrium quantities are positive or not ${ }^{43}$. Finally, whenever the consumers of all the integrating regions can afford consumption at the free-trade equilibrium price, the numerator and the denominator of the above expression coincide and $\varkappa_{\left\{n: \bar{p} \leq p_{0}^{n}\right\}}=1$. Whenever it is not the case, $\varkappa_{\left\{n: \bar{p} \leq p_{0}^{n}\right\}}>1$. Then:

Proposition 8 For $\mathbf{N}=\{1, \ldots, N\}$, if $\exists n, n^{\prime} \in \mathbf{N}: \frac{A_{n}}{B_{n}} \neq \frac{A_{n^{\prime}}}{B_{n^{\prime}}}$, then the regional integration of autarkic expectationally stable economies can be expectationally unstable. It is more likely so, the larger the disparity in the willingnesses to pay across regions.

Proof. See appendix 5 .

In the next section, we extend the conclusions obtained for the linear class of economies $\mathbf{N}$, to the non-linear class of economies $\mathbf{M}$.

\section{Integration of Non-linear Economies}

In this section, we explore the robustness of the conclusions of the previous section when the integrating regions are in the non-linear class $\mathbf{M}=\{1, \ldots, M\}$. The following condition guarantees that, with the appropriate boundary behaviour, the equilibria are unique (both autarkic and regionally integrated) and therefore (globally) determinate:

Condition (A.1.): $\forall p \in\left[0, p_{0}^{m}\right), D_{m}^{\prime}(p)<0, S_{m}^{\prime}(p)>0 ; p_{0}^{m} \equiv \min \left(D_{m}\right)^{-1}(0)>$ $0 ; S_{m}(0)=0 ; D_{m}(),. S_{m}(.) \in C^{1} ; \forall m \in \mathbf{M}$.

Notice that (A.1.) does not restrict the second derivatives of the supply and demand schedules, and also that $S_{m}^{\prime}()>$.0 implies that the underlying regional costs are convex.

Uniqueness of the regionally integrated PFE price $\bar{p}$ then follows from (A.1.), from appropriate boundary conditions ${ }^{44}$ and from the cobweb function being

\footnotetext{
${ }^{43}$ Actually, this is just a useful 'trick' that simplifies the proofs and allows an enlightening comparison with the results of proposition 6 above.

${ }^{44}$ The boundary conditions are: $\sum_{m}\left[D_{m}(0)-S_{m}(0)\right]>0$, and, for a small $\varepsilon>0$ : $\sum_{m}\left[D_{m}\left(\max _{m} p_{0}^{m}-\varepsilon\right)-S_{m}\left(\max _{m} p_{0}^{m}-\varepsilon\right)\right]<0$
} 
decreasing in the relevant price domain:

$$
\varphi^{\prime}(p)=\frac{\sum_{m} S_{m}^{\prime}(p)}{\sum_{m: p \leq p_{0}^{m}} D_{m}^{\prime}(p)}<0, \forall p \in\left[0, \max _{m} p_{0}^{m}-\varepsilon\right]
$$

To avoid the type of inconsistencies discussed in the previous section, we adopt a 'local eductive viewpoint', choosing a CK initial price restriction 'close' to the PFE price (in a neighbourhood around it), $p_{0} \in N_{\epsilon}(\bar{p})=(\bar{p}-\epsilon, \bar{p}+\epsilon)$. Whenever the learning process converges to it, we will say that the equilibrium is (locally) strongly rational (LSR). Since $\bar{p}$ is locally determinate, applying the implicit function theorem to the market clearing equation $D(\bar{p})=S(\bar{p})$, we obtain the following condition characterizing the learning dynamics:

$$
\lim _{\tau \rightarrow \infty}\left(p_{\tau}-\bar{p}\right)=\left(\frac{S^{\prime}(\bar{p})}{D^{\prime}(\bar{p})}\right)^{\tau}\left(p_{0}-\bar{p}\right)=0 \Leftrightarrow\left|\varphi^{\prime}(\bar{p})\right|=\left|\frac{S^{\prime}(\bar{p})}{D^{\prime}(\bar{p})}\right|<1
$$

According to the result (ii) of proposition 2 above. Since our purpose is to relate the condition for the expectational stability of the regionally integrated equilibrium to the autarkic stability ones, we can expand it as:

$$
\varphi^{\prime}(\bar{p})=\frac{\sum_{m} S_{m}^{\prime}(\bar{p})}{\sum_{m: \bar{p} \leq p_{0}^{m}} D_{m}^{\prime}(\bar{p})}=\varkappa_{\left\{m: \bar{p} \leq p_{0}^{m}\right\}} \sum_{m} \alpha_{m} \frac{D_{m}^{\prime}\left(\bar{p}_{m}\right)}{D_{m}^{\prime}(\bar{p})} \frac{S_{m}^{\prime}(\bar{p})}{S_{m}^{\prime}\left(\bar{p}_{m}\right)} \varphi_{m}^{\prime}\left(\bar{p}_{m}\right)
$$

Where the $\alpha_{m} \geq 0, \forall m: \sum_{m} \alpha_{m}=1$ represent the relative (to the world) demand elasticities of each of the integrating economies evaluated at the PFE price $\bar{p}$. The autarky PFE price of each $m$ region is denoted $\bar{p}_{m}$. The factor $\varkappa_{\left\{m: \bar{p} \leq p_{0}^{m}\right\}} \equiv \frac{\sum_{m} D_{m}^{\prime}(\bar{p})}{\sum_{m: \bar{p} \leq p_{0}^{m}} D_{m}^{\prime}(\bar{p})}$ has exactly the same interpretation as in the linear case: it accounts for differences in the maximal willingnesses to pay across the integrating regions, now in the non-linear class.

The first result extends proposition 3 to non-linear economies:

Proposition 9 The expectational stability of the M-replica Home economy in the non-linear class $\mathbf{M}$ obtains under the same conditions it does in the Home non-linear economy.

Proof. See appendix 5.

The importance of this proposition stems in recognizing that it is not nonlinearities per se that matter, but heterogeneity. Being it absent, the $M$ replica of the Home economy does not even create a redistributional conflict among producers, because the open economy price coincides with the autarkic one. However, the conclusions of propositions 4 and 5 do not extend to the nonlinear class. Figures 6 and 7 below illustrate, respectively, the reasons of such failures. In figure 6 , as new producers enter the Home market, the equilibrium price decreases at a higher rate than entry does, because the elasticity of demand increases more than proportionately (price-varying elasticity). Then, opening 
the Home market to Foreign producers may end up stabilizing expectations ${ }^{45}$.

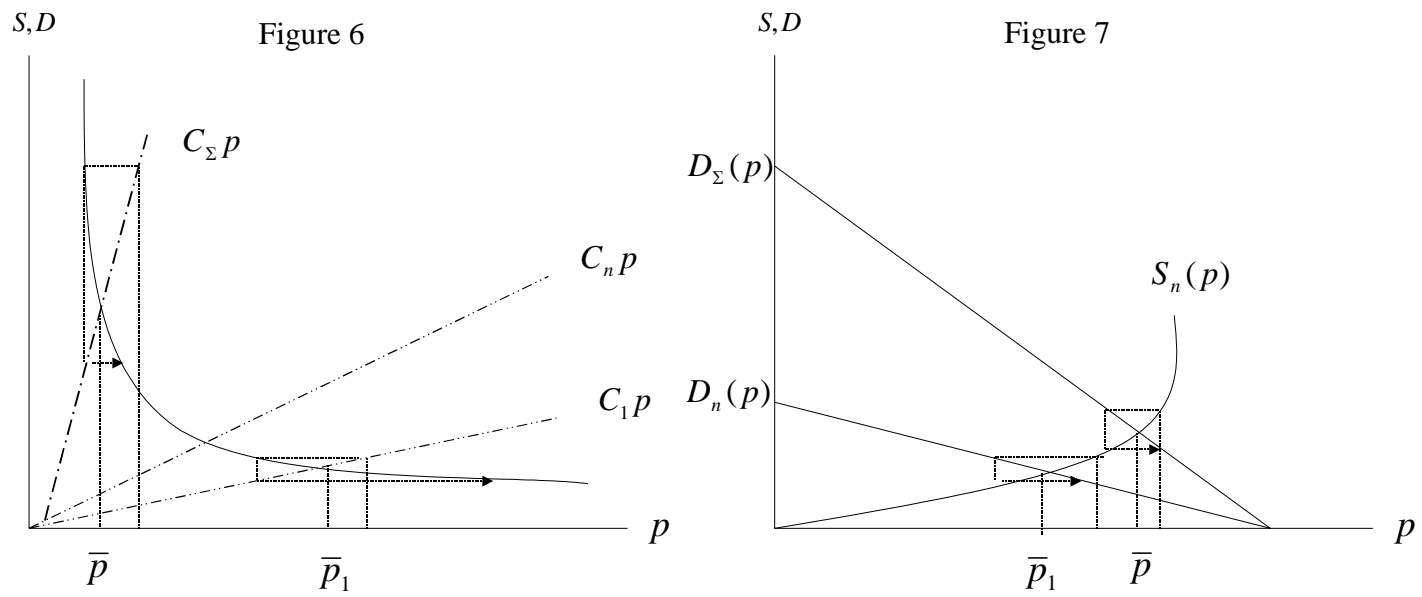

In figure 7, as the Home producers pregressively sell in Foreign markets, the effective demand they face increases and so will the equilibrium price. But if higher sales entail progressively lower marginal costs, the supply elasticity may considerably increase and the new price equilibrium becomes expectationally unstable. Then, new markets may actually destabilize expectations. The convexity of aggregate supply in picture 7 implies that marginal costs increase at a decreasing rate with units produced. This is rather unintuitive in this model with no sunk costs. Rather, if we assume that marginal costs increase at an increasing rate (ex. because of the decreasing returns to some fixed factor in the short run) the supply locii will be concave and new markets stabilize producers' expectations.

Both propositions, 4 and 5, fail to generalize because the comparative statics excercise ultimately depends on the magnitude of the response of farmers' reactions to structural changes, like an increase in foreign competition, or having access to new markets. That magnitude is governed by the second derivative of the supply (and demand) schedule(s). Requiring (supply and) demand schedules to be concave, and with equal marginal willingnesses to pay $\left(p_{0}^{m}=p_{0}\right.$, $\forall m \in \mathbf{M}$ ), allows a straightforward generalization to non-linear schedules of both propositions:

Proposition 10 Suppose that in addition to condition (A.1.), $D_{m}^{\prime \prime}()<0,. S_{m}^{\prime \prime}()<$. 0 , and $p_{0}^{m}=p_{0}, \forall m \in \mathbf{M}$. Then: (i) Proposition 4 extends to the non-linear class, (ii) Proposition 5 extends to the non-linear class.

Proof. See appendix 5.

\footnotetext{
${ }^{45}$ The problem is connected to the convexity of demand, which in usual Cournot games, prevents the players' reaction functions from being downward sloping, or, players' strategies from being strategic substitutes. See Vives [26], chapters 2 and 4 for a comprehensive explanation.
} 
Intuitively the proposition states that, whenever both demand and supply schedules are (globally) concave, opening new markets favours expectational coordination whereas opening the Home market to Foreign competitors is detrimental, even for integrating regions in the non-linear class $\mathbf{M}$.

If we extend proposition 6 to the non-linear class of economies, our main result states that:

Proposition 11 If there are no differences in the maximal willingnesses to pay across regions, then the regional integration of $M$ autarkic expectationally stable economies can result in an expectationally unstable PFE price.

Proof. See appendix 5.

This result is striking because it does not need any of the standard singlecrossing assumptions which are typical of these comparative statics exercises. Intuitively, it states that although regional integration stabilizes autarky prices across regions, it can destabilize producers' expectations, rendering more compelling the necessity of an 'exogenous price intervention' than it was in the autarkic regime. Notice that the non-linear class of economies allows one to reconcile the results of proposition 6 with Guesnerie's [14] adduced general intuition (GI2) that heterogeneity is detrimental to expectational coordination. In this sense, it provides an open economy interpretation of the forces behind: because economic integration equalizes pre-trade prices across regions, it creates a redistributional conflict between heterogeneous producers, who respond in proportion of their differences. Since the higher the degree of heterogeneity in the responses is, the more difficult it becomes for them to individually forecast, it is intuitive that the likelihood of successful coordination (on the open economy PFE price) decreases. Or stated more formally, as expectational stability is characterized by a condition on first derivatives, the consequences of policy changes upon it are characterized by a condition on second derivatives. Second derivatives precisely measure the magnitude of the individual reactions to the policy change which, because of its redistributive nature, embodies expectations of a different sign. Hence, the result of the proposition. Finally, notice that the same explanation holds for propositions 7 and 8 , because differences in consumers' willingnesses to pay allow differences in the magnitude of producers' reactions across regions in real cost terms, i.e. in costs per unit of regional purchasing power.

There remains to show that in the class of economies considered, regional integration actually equalizes (stabilizes) autarky equilibrium prices across regions:

Proposition $12 \bar{p} \in\left[\min _{m} \bar{p}_{m}, \max _{m} \bar{p}_{m}\right]$

Proof. See appendix 5.

For the sake of completeness, we let the reader remark that when differences in the maximal willingnesses to pay are allowed across economies in the nonlinear class $\mathbf{M}$, the PFE price of the regional integration is more difficult to 
learn than when they are absent. The proof follows the steps of proposition 8 and is immediate once we notice that:

$$
\varphi^{\prime}(\bar{p})=\varkappa_{\left\{m: \bar{p} \leq p_{0}^{m}\right\}} \sum_{m} \alpha_{m} \varphi_{m}^{\prime}(\bar{p})
$$

With $\varkappa_{\left\{m: \bar{p} \leq p_{0}^{m}\right\}} \geq 1$ as before, and with exactly the same interpretation.

\section{Coordination and Welfare}

An important rationale motivating open economy excercises are welfare considerations. In this section we will study this more traditional rationale for opening our partial equilibrium economies and relate it to the coordinational considerations studied in the previous sections. However, the nature of the exercise is necessarily from an ex-ante viewpoint (before integration takes place) and can be described as follows: Suppose that a given economy is considering with which country to integrate among those in a given class. A possible evaluation criterion is welfare, disregarding coordinational issues. Another evaluation criterion is expectational coordination. If we compare the reccomendations of both, do they coincide? The answer to this precise question wants to illustrate Guesnerie's [12] described trade-off, also present in this open economy exercise: standard efficiency gains associated with economic integration have to be weighted against the diminished plausibility of the PFE price in terms of its predictability after integration, as advanced by proposition 11. Nevertheless, the important caveat mentioned at the end of this section compromises any general conclusion beyond the simple exercise performed below.

Consider the linear class of economies where farmers face the same aggregate demand function, $D_{n}(p)=D(p), \forall n$, but differ in their cost structures across regions. The integrated economy will be more efficient than the autarkic ones if we measure efficiency by the net change in the Marshallian aggregate surplus (net producers' profits plus net consumers' surplus) and this change is positive ${ }^{46}$. The increase in welfare from integration for a given $n$ region is then defined by ${ }^{47}$ :

$$
\begin{aligned}
\Delta W_{n} & \equiv W_{n}^{*}-\bar{W}_{n}=\Delta C S_{n}-\Delta \Pi_{n} \\
& =\int_{p^{*}}^{\bar{p}_{n}} D_{n}(p) d p-\int_{p^{*}}^{\bar{p}_{n}} S_{n}(p) d p
\end{aligned}
$$

It can be seen that a conflict exists between the consumers and the producers of each of the integrating economies. The economy with the relatively more

\footnotetext{
${ }^{46}$ Since there are no general equilibrium effects (because there is no trade as only one product is considered), two economies in the class considered here have an incentive to integrate when appropriate redistributional schemes are implemented. For a more detailed discussion, see Mas-Colell et al. [19], section 10.E.

${ }^{47}$ We slightly change notation relative to the previous sections: now $p^{*}$ (instead of $\bar{p}$ ) denotes the integration equilibrium price, while $\bar{p}_{n}$ still denotes the autarkic equilibrium price of region $n$.
} 
performant producers ${ }^{48}\left(\max _{n} C_{n}\right)$ experiences an increase in profits $\left(\Delta \Pi_{n}>\right.$ 0 ) from selling abroad part of their production at a price $p^{*}$ higher than the autarkic one $\bar{p}_{n}$. This increase in the price damages the consumers living in that region, who see their consumer surplus eroded relative to the autarkic situation, $\Delta C S_{n}<0$. The converse happens in the region with the least performant producers $\left(\min _{n} C_{n}\right)$. But, the aggregate surplus increases after integration in each of the integrating economies ${ }^{49}$ :

$$
\begin{aligned}
\Delta W_{n} & =\int_{p^{*}}^{\bar{p}_{n}}[A-B p] d p-\int_{p^{*}}^{\bar{p}_{n}}\left[C_{n} p\right] d p \\
& =\left[\frac{A p}{2}\left\{2-\frac{B+C_{n}}{A} p\right\}\right]_{p^{*}}^{\bar{p}_{n}}=\frac{B+C_{n}}{2}\left[\bar{p}_{n}-p^{*}\right]^{2}>0, \forall n
\end{aligned}
$$

Because of this fact, we can assume that national (internal lump-sum ex-post) transfer schemes exist that are able to (more than) compensate the adversely affected party. This is always possible in this partial equilibrium framework, and everybody can be made strictly better off after integration ${ }^{50}$.

From this ex-ante welfare evaluation criterion, a given economy in the linear class would ideally choose an integration partner with which the increase in the net aggregate surplus is maximized. Region $\mathrm{H}$ must decide with which of the two region types ( $\mathrm{F}$ or $\mathrm{A}$ ) would it integrate, assuming that the producers in region $\mathrm{F}$ are more performant than those in the $\mathrm{H}$ region, while those in region A are less:

$$
+\infty>C_{F}>C_{H}>C_{A}>0
$$

Call the resulting integrated equilibrium prices $p_{H+F}^{*}$ and $p_{H+A}^{*}$. From the analytic expression of the net welfare gains in region $n, \Delta W_{n}$, we can see that

$$
0 \in \arg \sup _{C_{A}: C_{A} \leq C_{H}} \Delta W_{H}^{H+A}=\frac{B+C_{H}}{2}\left[\bar{p}_{H}-p_{H+A}^{*}\left(C_{A}\right)\right]^{2}
$$

Because, given the autarky price in the home region $\bar{p}_{H}$, the largest possible value of the integrated economy equilibrium price $p_{H+A}^{*}$ is obtained when the less performant among the abroad regions (A) is selected, i.e. as $C_{A} \longrightarrow 0$. Since the home region $(\mathrm{H})$ is more efficient, autarky prices are going to be lower: $p_{H+A}^{*}-\bar{p}_{H}>0$. As this difference is maximal whenever $C_{A} \longrightarrow 0$, denote by $\bar{\omega}$

\footnotetext{
${ }^{48}$ Note that with the specified cost structures, $\partial_{C_{f}} C\left(q_{f}, f\right)=-\left(\frac{q_{f}}{\sqrt{2} C_{f}}\right)^{2}<0$. Therefore, higher values of the cost parameter $C_{f}$ correspond to lower production costs, and to a relatively more performant production technique.

${ }^{49}$ Where $C_{\Sigma}=\sum_{n} C_{n}$ denotes the parameter of the total cost function in the integrated economy $C_{\Sigma}(q)$. Notice that, with an abuse of notation, we denote both a given integrating region and the summation subindex by $n$.

${ }^{50}$ Individual lump-sum transfer schemes could have been implemented in the way proposed by Dixit and Norman [6] (sec. 3.2.), as this partial equilibrium economy can be considered as a particular case of the general equilibrium economy they consider.
} 
its maximum value:

$$
\bar{\omega}=\lim _{C_{A} \longrightarrow 0} p_{H+A}^{*}\left(C_{A}\right)-\bar{p}_{H}=\frac{A}{B+\frac{C_{H}}{2}}-\frac{A}{B+C_{H}}>0
$$

Now, we look, among the foreign economies (F) that are more efficient than the home region, whether there is one that allows the home region to attain this same level of welfare $\Delta W_{H}^{H+A}(\bar{\omega})=\frac{B+C_{H}}{2}[\bar{\omega}]^{2}$. Since the foreign economy $(\mathrm{F})$ is more efficient, the integrated economy equilibrium price will be lower than the autarky equilibrium price at home $(\mathrm{H}), \bar{p}_{H}-p_{H+F}^{*}>0$. Then our problem can be stated formally as:

$$
\exists C_{F}: \bar{p}_{H}-p_{H+F}^{*}\left(C_{F}\right)>\bar{\omega}
$$

To prove this statement, we are going to proceed as follows: first, we are going to show that there exists a foreign region with a cost function parameter $\bar{C}_{F}$ such that the home economy reaches the level of welfare $\Delta W_{H}^{H+A}(\bar{\omega})$. Then, we are going to show that there is a set of more performant foreign regions, the integration of home with which yields strictly larger welfare gains. Finally, we show that as the home region becomes more efficient, it also becomes increasingly difficult to find such an F-region, in the sense that the 'measure' of the set of F-regions the integration with which yields larger expected welfare gains for the H-region, becomes close to zero.

First, $\bar{C}_{F}=C_{H}\left(2+\frac{C_{H}}{B}\right)$ satisfies the equation ${ }^{51}$ :

$$
\bar{p}_{H}-p_{H+F}^{*}\left(\bar{C}_{F}\right)=\bar{\omega} \Longrightarrow \Delta W_{H}^{H+F}(\bar{\omega})=\Delta W_{H}^{H+A}(\bar{\omega})
$$

Second, geometrically notice that $\bar{C}_{F}+C_{H}=\tan \bar{\theta}_{F+H}$. Provided that the home economy has an aggregate cost parameter strictly bounded from above, $C_{H}<+\infty$, and that it is not 'too expectationally unstable', $\frac{C_{H}}{B}<$ $M<+\infty$, then $\bar{C}_{F}+C_{H}=C_{H}\left(3+\frac{C_{H}}{B}\right)$ will also be strictly bounded above: $\arctan C_{H}\left(3+\frac{C_{H}}{B}\right)=\bar{\theta}_{F+H}<\frac{\pi}{2}=\arctan (+\infty)$. By continuity, there will exist a $\delta>0: \bar{\theta}_{F+H}<\bar{\theta}_{F+H}+\delta<\frac{\pi}{2}$ which will correspond to a foreign region with an aggregate cost parameter $C_{\bar{F}}<+\infty: C_{\bar{F}}+C_{H}=\tan \left[\bar{\theta}_{F+H}+\delta\right]=$ $C_{H}\left(3+\frac{C_{H}}{B}\right)+\Delta C$ and that will generate a strictly larger welfare gain for the home economy:

$$
\Delta W_{H}^{H+\bar{F}}>\Delta W_{H}^{H+F}(\bar{\omega}) \Longleftrightarrow \Delta C>0
$$

\footnotetext{
${ }^{51}$ For the class of linear economies considered with identical aggregate demand, the Fregion with a value of the aggregate cost parameter $\bar{C}_{F}$ that satisfies the above equation, must equivalently satisfy the condition:

$$
\frac{B+\bar{C}_{F}}{B+C_{H}}=\left.\frac{B+C_{H}}{B+C_{A}}\right|_{C_{A}=0}
$$

Whenever this condition is respected, the welfare gains for the home region from integrating with a more efficient $(F)$ or with a less efficient $(A)$ economy are the same, for economies in
} the linear class considered. 
Which is true by construction. Therefore, home integration with an F-region characterized by an aggregate cost parameter $C_{\bar{F}}$ displays strictly larger welfare gains than with the best possible integration partner in the set of A-regions.

Finally, under the just stated conditions, there exists an infinity of foreign regions $(\mathrm{F})$ that satisfy this condition, but the size of the set becomes smaller the more efficient the home region is, i.e. the larger the value of the parameter $C_{H}$. If we put a uniform probability measure on $\left[0, \frac{\pi}{2}\right]$ we can interpret the expression

$$
1-\mu\left[\bar{\theta}_{F+H}\right]=\int_{\bar{\theta}_{F+H}}^{\frac{\pi}{2}} \frac{2}{\pi} d v=1-\frac{\arctan C_{H}\left(3+\frac{C_{H}}{B}\right)}{\frac{\pi}{2}}
$$

as the likelihood of finding one F-region the integration with which provides higher welfare gains for the home region than integration with the best candidate in the set of A-regions. Then, from $\lim _{C_{H} \rightarrow+\infty}\left\{1-\mu\left[\bar{\theta}_{F+H}\right]\right\}=0$ we conclude that the more efficient the home region is, the lower the probability of finding an F-region the integration with which will yield the same welfare gains for home than integration with the best candidate A-region (all relatively less performant). Alternatively, the more performant the home region is, the smaller the size of the set of those F-regions the integration with which provides home with higher welfare gains than integration with the best candidate in the set of A-regions.

Now, the important point to be noted about this ex-ante welfare evaluation of the potential partner with which to integrate is that, the less performant the integrating partner is (the smaller the value of the aggregate cost parameter $C)$, the easier the coordination upon the perfect foresight equilibrium of the integrated economy. And conversely. For a strictly finite value of $C_{H}$, we also see that the likelihood of finding such an F-region integration partner decreases with the 'degree of expectational instability' of the home region, as measured by $\frac{C_{H}}{B}$. This can be seen immediately from the fact that:

$$
\partial_{B}\left\{1-\mu\left[\bar{\theta}_{F+H}(B)\right]\right\}=-\frac{2}{\pi} \partial_{B} \bar{\theta}_{F+H}(B)=\frac{\frac{2}{\pi}\left(\frac{C_{H}}{B}\right)^{2}}{1+C_{H}^{2}\left(3+\frac{C_{H}}{B}\right)^{2}}>0
$$

Therefore, the higher the degree of expectational stability of the home economy (the higher the value of $B$, the lower the value of $\frac{C_{H}}{B}$ ), the higher the likelihood of finding an economy in the F-class the integration with which yields strictly larger welfare gains than integration with an economy in the A-class. Recall that regions in the A-class are those expectationally more stable than the home economy because they face the same aggregate demand (and therefore, the same value of the elasticity of demand $B$ ) but operate with higher costs: $\frac{1}{C_{H}}<$ $\frac{1}{C_{A}}, \forall q$. Stated otherwise, if the purpose of Home economic integration is to maximize the welfare gains, relatively more performant regions will be preferred (F-regions will be preferred to A-regions), and government restrictions will be most likely called for to coordinate upon the equilibrium price of the resulting 
integrated economy. However, if the objective of Home economic integration is expectational coordination, relatively less performant regions will be preferred (A-regions will be preferred to F-regions). This is a surprising conclusion, the robustness of which remains to be ascertained ${ }^{52}$.

A caveat is in order. When the PFE price is not the unique rationalizable expectations equilibrium price, the aggregate surplus need not be the appropriate evaluation criterion in welfare terms. The reason is that it is based on the difference in welfare terms between the two Nash equilibrium prices (autarkic and integration) disregarding whether they can be educed or not. A more appropriate criterion in welfare terms would necessitate of computing producers' welfare when the set of rationalizable expectations equilibria is not a singleton. A solution to this serious problem (selection among a continuum of rationalizable equilibria) is beyond the scope of the present work. Therefore, a general conclusion along the lines of Guesnerie's [12] assertion mentioned in the beginning of the section is conditional to it.

\section{Conclusion}

In this work we have departed from the modern macroeconomics literature, which since the 70s, has devoted increasing effort to the assumptions justifying the implementation of a REE solution. Learning constitutes the current paradigm. Adopting the eductive learning viewpoint, we have related free-trade expectational stability conditions to autarkic ones, and provided an open economy natural interpretation to the elasticities condition identified by Guesnerie [11]. In the class of one-dimensional partial equilibrium models where the price is determined by the price expectations of infinitesimal but heterogeneous producers, trade captures the standard increase in product market competition complemented by its impact on producers' expectations ${ }^{53}$. As new entrants gain access to the Home market, the relative scarcity of the home produced commodity decreases, intensifying competition and lowering the price and profits of home producers, compelling their forecasts to increasingly rely on the strategic component of what do others expect, so undermining expectational coordination (adverse effect of a high supply elasticity). On the contrary, as new markets are available to the home producers, the relative scarcity of the home produced commodity increases leading both to higher prices and profits. As home competition is relaxed, so is the weight of the strategic component on producers' forecasts, easening expectational coordination (favourable effect of a high demand elasticity). Overall, policy changes that alter the competitive con-

\footnotetext{
${ }^{52}$ What seems crucial for the argument to extend to non-linear schedules is the existence of a finite maximal willingness to pay for the good.

${ }^{53}$ Recent contributions on firm heterogeneity in a general equilibrium setup have rather focused on the beneficial impact of trade on aggregate productivity through the improved labor allocation that the existence of better opportunities abroad entails. These contributions abstract from the standard increase in competition channel. See Bernard et al. [2] for a ricardian model with endogenous mark-ups, or Melitz [20] for a monopolistically competitive model where the number of varieties produced is endogenous.
} 
ditions have an impact on producers' ability to decide on a course of action, as measured by its impact on the accuracy of their forecasts. In standard classical and new trade theory models this aspect is absent, because the implicit action of the Walrasian auctioneer provides the invisible though necessary coordinational device.

As well, we have provided some new insights on the classical issue of tradeinduced efficiency gains, and shown that the same spatial differences in consumers' valuations or in price-varying elasticities that are detrimental to expectational stability, create the necessary spatial heterogeneity for the standard gains-to-trade argument to work (spatial price equalization or stabilization). Then, as spatial price stabilization destabilizes producers' price expectations, an exogenous intervention at the international level is justified since it allows the integrating regions to fully reap the (standard) benefits of trade. To illustrate this point, we have compared the expectational coordination objective to a traditional 'welfare gain' objective. We have shown that the maximal welfare gain for an autarkic expectationally stable region in the linear class obtains when integrating with an unstable region, compromising the objective of expectational coordination after integration. This example provides some precise content in an international economy setup to the trade-off noticed by Guesnerie [12], i.e. that the standard efficiency gains associated with economic integration must be weighted against the consequent increase in the plausibility of multiplicity of (rationalizable expectations) equilibria. However we also pointed out that this conclusion sidesteps the issue of evaluating equilibrium outcomes in welfare terms when the expectational stability test fails ${ }^{54}$. As such, no general conclusion can be taken before tackling the selection problem among rationalizable equilibria.

Although a natural extension would be to study whether the conditions for the eductive stability of the equilibrium in an open economy can be related to the basic theorems of international trade, a first difficulty stems in recognizing that most of such trade theorems concern comparative statics questions in a general equilibrium set up. Yet, most of the conclusions on the eductive stability literature relate to partial equilibrium economies ${ }^{55}$. Nevertheless, we hope to have provided insights that may prove fruitful in studying markets where structural changes that increase the heterogeneity of the system can be tied to increased volatility or to the emergence of multiple equilibria.

\footnotetext{
${ }^{54}$ In this sense, the application of continuous random selections over the set of rationalizable expectations equilibria, along the lines of Allen et al. [1], seems a necessary step in making progress through a meaningful gains-to-trade evaluation criterion meeting the learning justification requirements.

55 General equilibrium applications of the eductive viewpoint are scarce. Some of them are Guesnerie [13], Guesnerie and Hens [15] and Ghosal [10]. Calvo and Guesnerie [4] provide a brief introduction.
} 


\section{References}

[1] Allen, B., J. Dutta and H. Polemarchakis (2002), "Equilibrium Selections", in Essays in Honor of E. Drandakis, G. Bitros Ed., Edward Elgar Publisher.

[2] Bernard, A.B., J.Eaton, J.B. Jensen and S. Kortum (2000), "Plants and Productivity in International Trade", NBER WP 7688.

[3] Bernheim, D. (1984), "Rationalizable Economic Behaviour", Econometrica $52: 1007-1028$.

[4] Calvo Pardo H. and R. Guesnerie (2004), "Eductive Stability in Sequential Exchange Economies: An Introduction", DELTA DP (forthcoming) and Ch.6 in Assessing Rational Expectations: Eductive tability in Economics, by R.Guesnerie Ed., MIT Press (forthcoming).

[5] Desgranges, G. and S. Gauthier (2003), "Learning a Rational Expectations Equilibrium when Information is Asymmetric", paper presented at the 2003 ESEM, Stockholm.

[6] Dixit, A.K. and V. Norman (1980), Theory of International Trade, Cambridge Economic Handbooks, Cambridge University Press.

[7] Evans, G. (1985), "Expectational Stability and the Multiple Equilibria Problem in Linear Rational Expectations Models", Quarterly Jornal of Economics $100: 147-157$.

[8] Evans, G. and S. Honkapohja (2000), "Expectations and the Stability Problem for Optimal Monetary Policies", mimeo.

[9] Evans, G. and S. Honkapohja (2001), Learning and Expectations in Macroeconomics, Princeton, Princeton University Press.

[10] Ghosal, S. (2003), "Sequential Coordination, Eductive Stability and Rationalizability", University of Warwick, mimeo.

[11] Guesnerie, R. (1992), "An Exploration of the Eductive Justifications of the Rational Expectations Hypothesis", American Economic Review 82 :12541278 .

[12] Guesnerie, R. (2000), "The Government and Market Expectations", DELTA DP 2000-15.

[13] Guesnerie, R. (2001), "Short-Run Expectational Coordination: Fixed versus Flexible Wages", Quarterly Journal of Economics :1115-1147.

[14] Guesnerie, R. (2002), "Anchoring Economic Predictions in Common Knowledge", Econometrica $70: 439-480$.

[15] Guesnerie, R. and T. Hens (2001), "Expectational Coordination in Sequential Exchange Economies", mimeo. 
[16] Hommes, C. and F. Wagener (2003), "Does Eductive Stability imply Evolutionary Stability?", mimeo.

[17] Hopenhayn, H. (1992), "Entry, Exit and Firm Dynamics in Long Run Equilibrium", Econometrica $60: 1127-1150$.

[18] Manski, C.F. (2003), "Inference on Expectations and Decisions", FisherSchultz Lecture of the 2003 Econometric Society European Meeting, Stockholm.

[19] Mas-Colell, A., M.D. Whinston and J.R. Green (1995), Microeconomic Theory, Oxford University Press.

[20] Melitz, M. (2003), "The Impact of Trade on Intra-Industry Reallocations and Aggregate Industry Productivity", Econometrica (forthcoming).

[21] Milgrom, P. and J. Roberts (1990), "Rationalizability, Learning and Equilibrium in Games with Strategic Complementarities", Econometrica 58: $1255-1277$.

[22] Muth, J. (1961), "Rational Expectations and the Theory of Price Movements", Econometrica $29: 315-335$.

[23] Novshek, W. (1980), "Cournot Equilibrium with Free Entry", Review of Economic Studies 47: 473-486.

[24] Pierce, D. (1984), "Rationalizable Strategic Behaviour and the Problem of Perfection", Econometrica 52 :1029-1050.

[25] Tan, T.C-C. and S. R. da Costa Werlang (1988), "The Bayesian Foundations of Solution Concepts of Games", Journal of Economic Theory 45 :370-391.

[26] Vives,X. (1999), Oligopoly Pricing: Old Ideas and New Tools, MIT Press.

\section{Appendix 0}

Proof of Proposition 6

Under the condition $\frac{A_{n}}{B_{n}}=\frac{A_{n^{\prime}}}{B_{n^{\prime}}}=\frac{A}{B}, \forall n, n^{\prime} \in \mathbf{N}$ the anchorage assumption is given by $p_{0}=\frac{A_{\Sigma}}{B_{\Sigma}}=\frac{A}{B}$. For parts (i),(ii) compute the limit $\lim _{\tau \rightarrow+\infty} p_{\tau}$ of the price sequence in proposition 3 after replacing $\left(-\frac{C_{\Sigma}}{B_{n_{0}}}\right)$ by $\left(-\frac{C_{\Sigma}}{B_{\Sigma}}\right)$, and $\frac{A_{n_{0}}}{B_{n_{0}}}$ by $\frac{A_{\Sigma}}{B_{\Sigma}}$. To prove part (iii) notice that $D(p)=\sum_{n} D_{n}(p)$ implies that $D^{\prime}(p)=\sum_{n} D_{n}^{\prime}(p) \leq 0$ by $D_{n}^{\prime}(p) \leq 0, \forall n$. Also, $S(p)=\sum_{n} S_{n}(p)$ implies that $S^{\prime}(p)=\sum_{n} S_{n}^{\prime}(p)>0$ by $S_{n}^{\prime}(p)>0, \forall n$. The linearity of the regional demand and supply schedules implies that: $D_{n}^{\prime}\left(p_{1}\right)=D_{n}^{\prime}\left(p_{2}\right), S_{n}^{\prime}\left(p_{1}\right)=S_{n}^{\prime}\left(p_{2}\right), \forall n$ and $\forall p_{1}, p_{2} \in\left[0, p_{0}\right), \forall p_{1}, p_{2} \in\left[p_{0},+\infty\right),$. From part (i) in proposition $2,\left|\varphi^{\prime}(p)\right|=$ 
$\left|\frac{S^{\prime}(p)}{D^{\prime}(p)}\right|<1, \forall p$. Expanding the sums and using the linearity, we can rewrite it as $\left|\varphi^{\prime}(p)\right|=\left|\sum_{n} \frac{D_{n}^{\prime}(p)}{D^{\prime}(p)} \frac{S_{n}^{\prime}(p)}{D_{n}^{\prime}(p)}\right|=\left|\sum_{n} \alpha_{n} \varphi_{n}^{\prime}(p)\right|<1, \forall p$. Since $\forall n, \alpha_{n} \geq 0$, $\sum_{n} \alpha_{n}=1$, the regional integration expectational stability condition is a convex combination of the autarkic expectational stability conditions. Therefore:

$$
\min _{n}\left|\varphi_{n}^{\prime}(p)\right| \leq\left|\varphi^{\prime}(p)\right|=\left|\sum_{n} \alpha_{n} \varphi_{n}^{\prime}(p)\right| \leq \max _{n}\left|\varphi_{n}^{\prime}(p)\right|
$$

implies that if the autarkically most unstable region is expectationally stable, so must the regional integration be:

$$
\max _{n}\left|\varphi_{n}^{\prime}(p)\right|<1 \Longrightarrow\left|\varphi^{\prime}(p)\right|<1
$$

That the converse is not true follows trivially from the convex combination set of inequalities above.Q.E.D.

\section{Appendix 1}

Derivation and properties of the cobweb function

Derivation of the cobweb function $\varphi\left(p ; C_{\Sigma}\right)=\max \left\{\varphi_{1}\left(p ; C_{\Sigma}\right), \varphi_{2}\left(p ; C_{\Sigma}\right)\right\}$ for the agricultural economy $n=1+2$, with:

$$
\begin{aligned}
\varphi_{1}\left(p ; C_{\Sigma}\right) & =\frac{A_{\Sigma}}{B_{\Sigma}}-\left(\frac{C_{\Sigma}}{B_{\Sigma}}\right) p \\
\varphi_{2}\left(p ; C_{\Sigma}\right) & =\frac{A_{2}}{B_{2}}-\left(\frac{C_{\Sigma}}{B_{2}}\right) p
\end{aligned}
$$

1) $\varphi($.$) is a continuous function: \varphi($.$) is a continuous function since it is$ composed by two linear functions which always intersect in the price domain. Denote by $p^{i}$ the price function at which both linear functions intersect, i.e. $\varphi_{1}\left(p^{i} ; C_{\Sigma}\right)=\varphi_{2}\left(p^{i} ; C_{\Sigma}\right)$. It will be equal to:

$$
p^{i}\left(C_{\Sigma}\right)=\frac{B_{2} A_{\Sigma}-A_{2} B_{\Sigma}}{\left(B_{2}-B_{\Sigma}\right) C_{\Sigma}}=\frac{A_{1} \bar{C}_{\Sigma}}{B_{1} C_{\Sigma}}
$$

With $\bar{C}_{\Sigma} \equiv A_{2}\left[\frac{B_{1}}{A_{1}}-\frac{B_{2}}{A_{2}}\right]$. The linear functions defining the intersecting price are well-defined, mapping $\varphi_{1}\left(p ; C_{\Sigma}\right):\left[0, \frac{A_{\Sigma}}{C_{\Sigma}}\right] \rightarrow\left[0, \frac{A_{\Sigma}}{B_{\Sigma}}\right]$ and $\varphi_{2}\left(p ; C_{\Sigma}\right):\left[0, \frac{A_{2}}{C_{\Sigma}}\right] \rightarrow$ $\left[0, \frac{A_{2}}{B_{2}}\right]$. To see that they always intersect, observe that $\varphi_{2}\left(0 ; C_{\Sigma}\right)-\varphi_{1}\left(0 ; C_{\Sigma}\right)=$ $\frac{A_{2}}{B_{2}}-\frac{A_{\Sigma}}{B_{\Sigma}}=\frac{B_{1}}{B_{\Sigma}}\left[\frac{A_{2}}{B_{2}}-\frac{A_{1}}{B_{1}}\right]>0$ and that $\varphi_{2}\left(\frac{A_{2}}{C_{\Sigma}} ; C_{\Sigma}\right)-\varphi_{1}\left(\frac{A_{2}}{C_{\Sigma}} ; C_{\Sigma}\right)=0-\frac{A_{\Sigma}}{B_{\Sigma}}+$ $\frac{C_{\Sigma}}{B_{\Sigma}} \frac{A_{2}}{C_{\Sigma}}<0$. Since both are linear, both are continuous. 
2) The cobweb function is a maximum: First observe that:

$$
\begin{aligned}
\varphi\left(\bar{p} ; \bar{C}_{\Sigma}\right) & =\max \left\{\varphi_{1}\left(\bar{p} ; \bar{C}_{\Sigma}\right), \varphi_{2}\left(\bar{p} ; \bar{C}_{\Sigma}\right)\right\} \\
& =\max \left\{\bar{p}\left(\bar{C}_{\Sigma}\right), \bar{p}\left(\bar{C}_{\Sigma}\right)\right\} \\
& =\bar{p}\left(\bar{C}_{\Sigma}\right)=\frac{A_{1}}{B_{1}}=p^{i}\left(\bar{C}_{\Sigma}\right)
\end{aligned}
$$

Meaning that there exists a value of the aggregate cost parameter for which the price equilibrium coincides with the common intesecting price, and therefore will be a point in the range of the cobweb function.

Now consider w.l.o.g. a $C_{\Sigma}=C_{\Sigma}^{\prime}>\bar{C}_{\Sigma}$, and denote by $\bar{p}^{\prime}$ the corresponding PFE price equilibrium. By definition, $\varphi\left(\bar{p}^{\prime} ; C_{\Sigma}^{\prime}\right)=\bar{p}^{\prime}$. Since $C_{\Sigma}^{\prime}>\bar{C}_{\Sigma}$ then $\varphi_{1}\left(\bar{p}^{\prime} ; C_{\Sigma}^{\prime}\right)=\bar{p}^{\prime}$ whereas $\varphi_{2}\left(\bar{p}^{\prime} ; C_{\Sigma}^{\prime}\right) \neq \bar{p}^{\prime}$. If the function is a maximum, it must be the case that $\varphi_{2}\left(\bar{p}^{\prime} ; C_{\Sigma}^{\prime}\right)<\bar{p}^{\prime}=\varphi_{1}\left(\bar{p}^{\prime} ; C_{\Sigma}^{\prime}\right)$.

Proof: It will be the case if the price at which both linear functions intersect is smaller than the equilibrium price, i.e. if $p^{i}\left(C_{\Sigma}^{\prime}\right)<\bar{p}^{\prime}$. Suppose that the opposite is true. Then:

$$
\begin{aligned}
\frac{A_{1} \bar{C}_{\Sigma}}{B_{1} C_{\Sigma}^{\prime}} & =p^{i}\left(C_{\Sigma}^{\prime}\right)>\bar{p}^{\prime}=\frac{A_{\Sigma}}{B_{\Sigma}+C_{\Sigma}^{\prime}} \Longleftrightarrow \\
A_{2} \frac{B_{1}}{A_{1}}-B_{2} & =\bar{C}_{\Sigma}>\frac{B_{1}}{A_{1}} \frac{C_{\Sigma}^{\prime} A_{\Sigma}}{B_{\Sigma}+C_{\Sigma}^{\prime}}=\frac{C_{\Sigma}^{\prime}\left(B_{1}+A_{2} \frac{B_{1}}{A_{1}}\right)}{B_{\Sigma}+C_{\Sigma}^{\prime}} \Longleftrightarrow \\
A_{2} \frac{B_{1}}{A_{1}} B_{\Sigma}-\left(B_{\Sigma}+C_{\Sigma}^{\prime}\right) B_{2} & =\bar{C}_{\Sigma} B_{\Sigma}-C_{\Sigma}^{\prime} B_{2}>C_{\Sigma}^{\prime} B_{1} \Longleftrightarrow \bar{C}_{\Sigma}>C_{\Sigma}^{\prime}
\end{aligned}
$$

A contradiction. Therefore, the cobweb function is a maximum. Q.E.D.

Using this fact and the intersecting price $p^{i}\left(C_{\Sigma}\right)$, we can also write the cobweb function as follows:

$$
\begin{aligned}
\varphi\left(p ; C_{\Sigma}\right) & =\max \left\{\varphi_{1}\left(p ; C_{\Sigma}\right), \varphi_{2}\left(p ; C_{\Sigma}\right)\right\} \\
& =\left\{\begin{array}{l}
\varphi_{2}\left(p ; C_{\Sigma}\right) \text { if } p \leq p^{i}\left(C_{\Sigma}\right) \\
\varphi_{1}\left(p ; C_{\Sigma}\right) \text { if } p \geq p^{i}\left(C_{\Sigma}\right)
\end{array}\right.
\end{aligned}
$$

A fact that follows from the observing that $\varphi_{2}\left(0 ; C_{\Sigma}\right)=\frac{A_{2}}{B_{2}}>\varphi_{1}\left(0 ; C_{\Sigma}\right)=\frac{A_{\Sigma}}{B_{\Sigma}}$ and that $\left|\partial_{p} \varphi_{2}\left(p ; C_{\Sigma}\right)\right|=\left|-\frac{C_{\Sigma}}{B_{2}}\right|>\left|\partial_{p} \varphi_{1}\left(p ; C_{\Sigma}\right)\right|=\left|-\frac{C_{\Sigma}}{B_{\Sigma}}\right|, \forall p \in\left[0, p_{\infty}\right)$ with $p_{\infty} \equiv\left(\varphi_{1}\right)^{-1}\left(0 ; C_{\Sigma}\right)$.

3) The cobweb function is (weakly) decreasing: We can conclude that the cobweb function is decreasing in its price domain $\partial_{p} \varphi\left(p ; C_{\Sigma}\right)<0$ from the fact that it is the maximum of two strictly decreasing linear functions $\partial_{p} \varphi_{n}\left(p ; C_{\Sigma}\right)<$ $0, n=1,2$. However $\varphi\left(p ; C_{\Sigma}\right)$ is not $C^{1}$ because it is a max function with a nondifferentiability point at the intersecting price $p^{i}\left(C_{\Sigma}\right)$.

4) Domain and Range of the cobweb function: Finally observe that since the cobweb function maps prices into prices with domain and range given by 


$$
\begin{aligned}
& \varphi\left(p ; C_{\Sigma}\right):\left[0, \max \left\{\left(\varphi_{1}\right)^{-1}(0),\left(\varphi_{2}\right)^{-1}(0)\right\}\right] \rightarrow\left[0, \max \left\{\varphi_{1}\left(0 ; C_{\Sigma}\right), \varphi_{2}\left(0 ; C_{\Sigma}\right)\right\}\right] \\
& \text { with } \max \left\{\left(\varphi_{1}\right)^{-1}(0),\left(\varphi_{2}\right)^{-1}(0)\right\}=\left(\varphi_{1}\right)^{-1}(0)=\frac{A_{\Sigma}}{C_{\Sigma}} \text { and } \max \left\{\varphi_{1}\left(0 ; C_{\Sigma}\right), \varphi_{2}\left(0 ; C_{\Sigma}\right)\right\}= \\
& \varphi_{2}\left(0 ; C_{\Sigma}\right)=\frac{A_{2}}{B_{2}} .
\end{aligned}
$$

Using that $\varphi\left(p ; C_{\Sigma}\right)$ is a decreasing function and the intersecting price $p^{i}\left(C_{\Sigma}\right)$ to know where the equilibrium price is, i.e.

$$
\begin{aligned}
& \varphi_{1}\left(p^{i} ; C_{\Sigma}\right)=\varphi_{2}\left(p^{i} ; C_{\Sigma}\right)>p^{i}\left(C_{\Sigma}\right) \Longrightarrow p^{i}\left(C_{\Sigma}\right)<\varphi\left(\bar{p} ; C_{\Sigma}\right)=\varphi_{1}\left(\bar{p} ; C_{\Sigma}\right)=\bar{p} \\
& \varphi_{1}\left(p^{i} ; C_{\Sigma}\right)=\varphi_{2}\left(p^{i} ; C_{\Sigma}\right)<p^{i}\left(C_{\Sigma}\right) \Longrightarrow p^{i}\left(C_{\Sigma}\right)>\varphi\left(\bar{p} ; C_{\Sigma}\right)=\varphi_{2}\left(\bar{p} ; C_{\Sigma}\right)=\bar{p} \\
& \varphi_{1}\left(p^{i} ; C_{\Sigma}\right)=\varphi_{2}\left(p^{i} ; C_{\Sigma}\right)=p^{i}\left(C_{\Sigma}\right) \Longrightarrow p^{i}\left(C_{\Sigma}\right)=\varphi\left(\bar{p} ; C_{\Sigma}\right)=\varphi_{1}\left(\bar{p} ; C_{\Sigma}\right)=\varphi_{2}\left(\bar{p} ; C_{\Sigma}\right)=\bar{p}
\end{aligned}
$$

This observation will be useful in the study of the second iterate of the cobweb function, in appendix 2. This concludes the description of the properties of the cobweb function.

\section{Appendix 2}

Derivation of $\varphi^{2}($.$) .$

Derivation of the second iterate of the cobweb function $\varphi^{2}(p)$ :

$$
\begin{aligned}
\varphi^{2}(p) & =\varphi(\varphi(p))=\max \left\{\varphi_{1}[\varphi(p)], \varphi_{2}[\varphi(p)]\right\} \\
& =\max \left\{\varphi_{1}\left[\max \left\{\varphi_{1}(p), \varphi_{2}(p)\right\}\right], \varphi_{2}\left[\max \left\{\varphi_{1}(p), \varphi_{2}(p)\right\}\right]\right\} \\
& =\max \left\{\min \left\{\left(\varphi_{1} \circ \varphi_{2}\right)(p),\left(\varphi_{1} \circ \varphi_{1}\right)(p)\right\}, \min \left\{\left(\varphi_{2} \circ \varphi_{2}\right)(p),\left(\varphi_{2} \circ \varphi_{1}\right)(p)\right\}\right\}
\end{aligned}
$$

Where the first equalities follow by definition, and the last one follows from the downward slopingness of the functions $\varphi_{n}($.$) so that \varphi_{n}\left[\max \left\{\varphi_{1}(p), \varphi_{2}(p)\right\}\right]=$ $\min \left\{\varphi_{n}\left(\varphi_{2}(p)\right), \varphi_{n}\left(\varphi_{1}(p)\right)\right\}$ for $n=1,2$. The different linear functions composing its definition are given by:

$$
\begin{aligned}
& \left(\varphi_{1} \circ \varphi_{2}\right)(p)=\frac{A_{\Sigma}}{B_{\Sigma}}+\frac{A_{2}}{B_{2}}\left(-\frac{C_{\Sigma}}{B_{\Sigma}}\right)+\left(-\frac{C_{\Sigma}}{B_{\Sigma}}\right)\left(-\frac{C_{\Sigma}}{B_{2}}\right) p \\
& \left(\varphi_{2} \circ \varphi_{1}\right)(p)=\frac{A_{2}}{B_{2}}+\frac{A_{\Sigma}}{B_{\Sigma}}\left(-\frac{C_{\Sigma}}{B_{2}}\right)+\left(-\frac{C_{\Sigma}}{B_{2}}\right)\left(-\frac{C_{\Sigma}}{B_{\Sigma}}\right) p \\
& \left(\varphi_{1} \circ \varphi_{1}\right)(p)=\frac{A_{\Sigma}}{B_{\Sigma}}+\frac{A_{\Sigma}}{B_{\Sigma}}\left(-\frac{C_{\Sigma}}{B_{\Sigma}}\right)+\left(-\frac{C_{\Sigma}}{B_{\Sigma}}\right)^{2} p \\
& \left(\varphi_{2} \circ \varphi_{2}\right)(p)=\frac{A_{2}}{B_{2}}+\frac{A_{2}}{B_{2}}\left(-\frac{C_{\Sigma}}{B_{2}}\right)+\left(-\frac{C_{\Sigma}}{B_{2}}\right)^{2} p
\end{aligned}
$$

Before proceeding, we can study the functions that compose $\varphi^{2}(p)$, which are $\varphi_{1}[\varphi(p)]$, and $\varphi_{2}[\varphi(p)]$. These two functions are well defined since they are the composition of a linear function and a continuous maximum fuction, mapping $\left(\varphi_{1} \circ \varphi\right)(p):\left[0,\left(\varphi_{1}\right)^{-1}(0)\right] \stackrel{\varphi}{\rightarrow}\left[0, \varphi_{2}\left(0 ; C_{\Sigma}\right)\right] \stackrel{\varphi_{1}}{\rightarrow}\left[\varphi_{1}\left[\varphi_{2}\left(0 ; C_{\Sigma}\right)\right], \varphi_{1}(0)\right]$ 
and $\left(\varphi_{2} \circ \varphi\right)(p):\left[0,\left(\varphi_{1}\right)^{-1}(0)\right] \stackrel{\varphi}{\rightarrow}\left[0, \varphi_{2}\left(0 ; C_{\Sigma}\right)\right] \stackrel{\varphi_{2}}{\rightarrow}\left[\varphi_{2}\left[\varphi_{2}\left(0 ; C_{\Sigma}\right)\right], \varphi_{2}(0)\right]$, since $\max \left\{\left(\varphi_{1}\right)^{-1}(0),\left(\varphi_{2}\right)^{-1}(0)\right\}=\left(\varphi_{1}\right)^{-1}(0), \max \left\{\varphi_{1}\left(0 ; C_{\Sigma}\right), \varphi_{2}\left(0 ; C_{\Sigma}\right)\right\}=$ $\varphi_{2}\left(0 ; C_{\Sigma}\right)$ and $\left(\varphi_{n}\right)^{\prime}()<0,. \forall n$. Therefore, the second iterate of the cobweb function maps $\varphi^{2}(p):\left[0,\left(\varphi_{1}\right)^{-1}(0)\right] \stackrel{\max \left\{\varphi_{1} \circ \varphi, \varphi_{2} \circ \varphi\right\}}{\rightarrow}\left[\max \left\{\varphi_{1}\left[\varphi_{2}\left(0 ; C_{\Sigma}\right)\right], \varphi_{2}\left[\varphi_{2}\left(0 ; C_{\Sigma}\right)\right]\right\}, \varphi_{2}(0)\right]$. Where $\max \left\{\varphi_{1}\left[\varphi_{2}\left(0 ; C_{\Sigma}\right)\right], \varphi_{2}\left[\varphi_{2}\left(0 ; C_{\Sigma}\right)\right]\right\}=\varphi_{1}\left[\varphi_{2}\left(0 ; C_{\Sigma}\right)\right]$.

Derivation of $p^{i \prime}$ and properties characterizing the function $\varphi^{2}($.

There is a (second) intesecting price, denoted $p^{i \prime}\left(C_{\Sigma}\right)$, that characterizes the second iterate of the cobweb function satisfying:

$$
\exists p^{i \prime}:\left(\varphi_{1} \circ \varphi\right)\left(p^{i \prime}\right)=\left(\varphi_{2} \circ \varphi\right)\left(p^{i \prime}\right)=\varphi^{2}\left(p^{i \prime}\right)
$$

And with the properties that:

1) It characterizes the second iterate of the cobweb function (toghether with $\left.p^{i}\right)$ as a piecewise linear function:

$$
\varphi^{2}(p)=\left\{\begin{array}{l}
\left(\varphi_{1} \circ \varphi\right)(p) \text { if } \varphi(p) \geq \varphi\left(p^{i \prime}\right) \Longleftrightarrow p \leq p^{i \prime} \\
\left(\varphi_{2} \circ \varphi\right)(p) \text { if } \varphi(p) \leq \varphi\left(p^{i \prime}\right) \Longleftrightarrow p \geq p^{i \prime}
\end{array}\right.
$$

Using the property $\left|\partial_{p} \varphi_{2}\left(p ; C_{\Sigma}\right)\right|>\left|\partial_{p} \varphi_{1}\left(p ; C_{\Sigma}\right)\right|$ and $\varphi^{\prime}()<$.0 . And as we showed in appendix 1 for the cobweb function, this intersecting price $p^{i \prime}$ also satisfies by definition and by $\varphi^{\prime}()<$.0 that if:

$$
\begin{aligned}
& \left(\varphi_{1} \circ \varphi\right)\left(p^{i \prime}\right)=\left(\varphi_{2} \circ \varphi\right)\left(p^{i \prime}\right)=\varphi^{2}\left(p^{i \prime}\right)>\varphi\left(p^{i \prime}\right) \Longrightarrow \varphi(\bar{p})=\varphi_{1}(\bar{p})>\varphi\left(p^{i \prime}\right) \Longleftrightarrow \bar{p}<p^{i \prime} \\
& \left(\varphi_{1} \circ \varphi\right)\left(p^{i \prime}\right)=\left(\varphi_{2} \circ \varphi\right)\left(p^{i \prime}\right)=\varphi^{2}\left(p^{i \prime}\right)<\varphi\left(p^{i \prime}\right) \Longrightarrow \varphi(\bar{p})=\varphi_{2}(\bar{p})<\varphi\left(p^{i \prime}\right) \Longleftrightarrow \bar{p}>p^{i \prime} \\
& \left(\varphi_{1} \circ \varphi\right)\left(p^{i \prime}\right)=\left(\varphi_{2} \circ \varphi\right)\left(p^{i \prime}\right)=\varphi^{2}\left(p^{i \prime}\right)=\varphi\left(p^{i \prime}\right) \Longrightarrow \varphi(\bar{p})=\varphi_{1}(\bar{p})=\varphi_{2}(\bar{p})=\varphi\left(p^{i \prime}\right) \Longleftrightarrow \bar{p}=p^{i \prime}
\end{aligned}
$$

2) Explicit expression for $p^{i \prime}$ :

To obtain an explicit expression for $p^{i \prime}\left(C_{\Sigma}\right)$ we can solve the equation $\left(\varphi_{1} \circ\right.$ $\varphi)\left(p^{i \prime}\right)=\left(\varphi_{2} \circ \varphi\right)\left(p^{i \prime}\right)$ yielding:

$$
C_{\Sigma} \varphi\left(p^{i \prime}\right)=\frac{A_{1}}{B_{1}} \bar{C}_{\Sigma} \Longleftrightarrow \varphi\left(p^{i \prime}\right)=p^{i}
$$

Where $p^{i} \equiv p^{i}\left(C_{\Sigma}\right)=\frac{A_{1}}{B_{1}} \frac{\bar{C}_{\Sigma}}{C_{\Sigma}}$ is the (first) intersecting price we derived in appendix 1. Given that if:

$$
\begin{aligned}
& \varphi\left(p^{i}\right) \leq p^{i} \Longrightarrow \varphi(p)=\varphi_{2}(p), \forall p \leq p^{i} \\
& \varphi\left(p^{i}\right) \geq p^{i} \Longrightarrow \varphi(p)=\varphi_{1}(p), \forall p \geq p^{i}
\end{aligned}
$$

and that by $\varphi^{\prime}()<$.0 we have:

$$
\begin{aligned}
& \varphi\left(p^{i \prime}\right)=p^{i} \geq \varphi\left(p^{i}\right) \Longleftrightarrow p^{i \prime} \leq p^{i} \\
& \varphi\left(p^{i \prime}\right)=p^{i} \leq \varphi\left(p^{i}\right) \Longleftrightarrow p^{i \prime} \geq p^{i}
\end{aligned}
$$


we can conclude from both that:

$$
\begin{aligned}
& \text { if } p^{i \prime} \leq p^{i} \Longrightarrow \varphi\left(p^{i \prime}\right)=\varphi_{2}\left(p^{i \prime}\right)=p^{i} \Longrightarrow p_{2}^{i \prime}=\varphi_{2}^{-1}\left(p^{i}\right) \\
& \text { if } p^{i \prime} \geq p^{i} \Longrightarrow \varphi\left(p^{i \prime}\right)=\varphi_{1}\left(p^{i \prime}\right)=p^{i} \Longrightarrow p_{1}^{i \prime}=\varphi_{1}^{-1}\left(p^{i}\right)
\end{aligned}
$$

With explicit formulas:

$$
\begin{aligned}
& p_{1}^{i \prime}\left(C_{\Sigma}\right)=\frac{A_{\Sigma}}{C_{\Sigma}}-\left(\frac{B_{\Sigma}}{C_{\Sigma}}\right) p^{i}\left(C_{\Sigma}\right) \\
& p_{2}^{i \prime}\left(C_{\Sigma}\right)=\frac{A_{2}}{C_{\Sigma}}-\left(\frac{B_{2}}{C_{\Sigma}}\right) p^{i}\left(C_{\Sigma}\right)
\end{aligned}
$$

So that the explicit expression for $p^{i \prime}\left(C_{\Sigma}\right)$ is:

$$
p^{i \prime}\left(C_{\Sigma}\right)=\max \left\{p_{1}^{i \prime}\left(C_{\Sigma}\right), p_{2}^{i \prime}\left(C_{\Sigma}\right), 0\right\}
$$

Proof: Because $\forall C_{\Sigma} \leq(\geq) \bar{C}_{\Sigma}$ we have that $p^{i}\left(C_{\Sigma}\right) \geq(\leq) p^{i \prime}\left(C_{\Sigma}\right)$ and by the argument above, $p^{i \prime}\left(C_{\Sigma}\right)=p_{2}^{i \prime}\left(C_{\Sigma}\right) \geq p_{1}^{i \prime}\left(C_{\Sigma}\right)\left(p^{i \prime}\left(C_{\Sigma}\right)=p_{1}^{i \prime}\left(C_{\Sigma}\right) \geq\right.$ $\left.p_{2}^{i \prime}\left(C_{\Sigma}\right)\right)$. (i) To see that $\forall C_{\Sigma} \leq(\geq) \bar{C}_{\Sigma}$ we have that $p_{1}^{i \prime}\left(C_{\Sigma}\right) \leq(\geq) p_{2}^{i \prime}\left(C_{\Sigma}\right)$ we remark that the only value of $C_{\Sigma}$ at which both functions $p_{2}^{i \prime}\left(C_{\Sigma}\right), p_{1}^{i \prime}\left(C_{\Sigma}\right)$ intersect is $C_{\Sigma}=\bar{C}_{\Sigma}$ so that $p_{2}^{i \prime}\left(\bar{C}_{\Sigma}\right)=p_{1}^{i \prime}\left(\bar{C}_{\Sigma}\right)$. At that point we also have:

$$
\partial_{C_{\Sigma}} p_{2}^{i \prime}\left(\bar{C}_{\Sigma}\right)=-\frac{1}{\bar{C}_{\Sigma}} \frac{A_{1}}{B_{1}}\left[1-\frac{B_{2}}{\bar{C}_{\Sigma}}\right]
$$

And:

$$
\partial_{C_{\Sigma}} p_{1}^{i \prime}\left(\bar{C}_{\Sigma}\right)=-\frac{1}{\bar{C}_{\Sigma}} \frac{A_{1}}{B_{1}}\left[1-\frac{B_{\Sigma}}{\bar{C}_{\Sigma}}\right]=\partial_{C_{\Sigma}} p_{2}^{i \prime}\left(\bar{C}_{\Sigma}\right)+\frac{A_{1}}{\left(\bar{C}_{\Sigma}\right)^{2}}
$$

So that at the unique point at which both functions intersect, we have $\partial_{C_{\Sigma}} p_{1}^{i \prime}\left(\bar{C}_{\Sigma}\right)>$ $\partial_{C_{\Sigma}} p_{2}^{i \prime}\left(\bar{C}_{\Sigma}\right)$ concluding that $\forall C_{\Sigma} \leq(\geq) \bar{C}_{\Sigma}$ we have that $p_{1}^{i \prime}\left(C_{\Sigma}\right) \leq(\geq) p_{2}^{i \prime}\left(C_{\Sigma}\right)$. (ii) Now, it is also true that the only value of $C_{\Sigma}$ at which each of the functions $p_{2}^{i \prime}\left(C_{\Sigma}\right), p_{1}^{i \prime}\left(C_{\Sigma}\right)$ intersects with $p^{i}\left(C_{\Sigma}\right)$ is $C_{\Sigma}=\bar{C}_{\Sigma}$. Therefore $p_{2}^{i \prime}\left(\bar{C}_{\Sigma}\right)=$ $p_{1}^{i \prime}\left(\bar{C}_{\Sigma}\right)=p^{i}\left(\bar{C}_{\Sigma}\right)$. Since at that point it is also true that $\partial_{C_{\Sigma}} p^{i}\left(\bar{C}_{\Sigma}\right)=-\frac{1}{\bar{C}_{\Sigma}} \frac{A_{1}}{B_{1}}$, by (i) it is true that $\partial_{C_{\Sigma}} p_{2}^{i \prime}\left(\bar{C}_{\Sigma}\right)=\partial_{C_{\Sigma}} p^{i}\left(\bar{C}_{\Sigma}\right)+\frac{A_{1} B_{2}}{B_{1}\left(\bar{C}_{\Sigma}\right)^{2}}$ and consequently $\partial_{C_{\Sigma}} p_{1}^{i \prime}\left(\bar{C}_{\Sigma}\right)>\partial_{C_{\Sigma}} p_{2}^{i \prime}\left(\bar{C}_{\Sigma}\right)>\partial_{C_{\Sigma}} p^{i}\left(\bar{C}_{\Sigma}\right)$. We can then conclude that $\forall C_{\Sigma} \leq(\geq$ ) $\bar{C}_{\Sigma}$ we have that $p^{i}\left(C_{\Sigma}\right) \geq(\leq) p^{i \prime}\left(C_{\Sigma}\right)$ as we wanted to show. Q.E.D.

3) Non-monotonicity of $p^{i \prime}$ :

Observe that:

$$
\begin{aligned}
& \partial_{C_{\Sigma}} p_{2}^{i \prime}\left(\bar{C}_{\Sigma}\right)>(\leq) 0 \text { if } \bar{C}_{\Sigma}<(\geq) B_{2} \equiv C_{\Sigma}^{2} \\
& \partial_{C_{\Sigma}} p_{1}^{i \prime}\left(\bar{C}_{\Sigma}\right)>0 \text { if } \bar{C}_{\Sigma}<B_{2}+B_{1} \equiv C_{\Sigma}^{1}
\end{aligned}
$$

Where the last row follows from a fact that will be used below again, but that we prove here: Suppose by contradiction that $\bar{C}_{\Sigma} \geq C_{\Sigma}^{1}$. Equivalently, $A_{2} \frac{B_{1}}{A_{1}}-B_{2} \geq$ 
$B_{\Sigma}$ or $B_{1}\left[\frac{A_{2}}{A_{1}}-1\right] \geq 2 B_{2}$. Since $A_{1} \geq A_{2}, \frac{A_{2}}{A_{1}} \leq 1$ and $0 \geq B_{1}\left[\frac{A_{2}}{A_{1}}-1\right] \geq 2 B_{2}$ violating the restriction $B_{2}>0$. Therefore $0 \leq \bar{C}_{\Sigma}<C_{\Sigma}^{1}$ and the slope of $p_{1}^{i \prime}$ at the point $C_{\Sigma}=\bar{C}_{\Sigma}$ can only be positive: The maximum of $p_{1}^{i \prime}$ will always be at a value of the aggregate cost larger than $\bar{C}_{\Sigma}$. The functional form of both functions displays a maximum in the aggregate cost domain $\mathbb{R}_{++}$.

Parameter values $C_{\Sigma}^{1}, C_{\Sigma}^{2}$ will be used below to characterize the learning dynamics.

4) Value of $p^{i \prime}$ in the analytic case studied by Guesnerie [11]:

If $\frac{A_{1}}{B_{1}}=\frac{A_{2}}{B_{2}}$ then $\bar{C}_{\Sigma}=0$ and $\varphi(p)=\varphi_{1}(p), \forall p$ and the intersecting price $p^{i}=0$. In consequence, $p^{i \prime}=\varphi_{1}^{-1}(0)=\frac{A_{\Sigma}}{C_{\Sigma}}>0$ and $\bar{p}=\bar{p}_{1} \in\left[p^{i}, p^{i \prime}\right]=\left[0, p_{1}^{i \prime}\right]$.

5) Defines a non-empty interval to which the perfect foresight equilibrium price $\bar{p}$ always belongs:

Proof: Consider the (first) intesecting price $p^{i}\left(C_{\Sigma}\right)$. We are going to show that the perfect foresight equilibrium price $\bar{p}$ must be contained in a non-empty interval between the two intersecting prices $p^{i}$ and $p^{i \prime}$. First suppose that:

$$
\varphi^{2}\left(p^{i \prime}\right)>\varphi\left(p^{i \prime}\right) \Longrightarrow \varphi(\bar{p})=\varphi_{1}(\bar{p})>\varphi\left(p^{i \prime}\right) \Longleftrightarrow \bar{p}<p^{i \prime}
$$

Now it can happen that $\bar{p}<p^{i}$ so that $\bar{p} \notin\left[p^{i}, p^{i^{i}}\right]$. But if $\bar{p}<p^{i}$ from the definition of $p^{i}$ we have that $\bar{p}=\varphi_{2}(\bar{p})$. A contradiction since $\bar{p}<p^{i \prime}$. Therefore, $\bar{p}>p^{i}$ and $\bar{p} \in\left[p^{i}, p^{i^{\prime}}\right]$. Second suppose that:

$$
\varphi^{2}\left(p^{i \prime}\right)<\varphi\left(p^{i \prime}\right) \Longrightarrow \varphi(\bar{p})=\varphi_{2}(\bar{p})<\varphi\left(p^{i \prime}\right) \Longleftrightarrow \bar{p}>p^{i \prime}
$$

Which leads to $\bar{p} \in\left[p^{i^{\prime}}, p^{i}\right]$ using the same reasoning. Finally, if:

$$
\varphi^{2}\left(p^{i \prime}\right)=\varphi\left(p^{i \prime}\right) \Longrightarrow \varphi(\bar{p})=\varphi_{1}(\bar{p})=\varphi_{2}(\bar{p})=\varphi\left(p^{i \prime}\right) \Longleftrightarrow \bar{p}=p^{i \prime}
$$

Then it must be the case by the same argument, that $\bar{p} \in\left[p^{i^{\prime}}, p^{i}\right]=\{\bar{p}\}$. Q.E.D.

Using some of these properties we can rewrite $\varphi^{2}(p)$ alternatively as follows:

$$
\begin{aligned}
& \varphi^{2}(p)= \begin{cases}\min \left\{\left(\varphi_{1} \circ \varphi_{2}\right)(p),\left(\varphi_{1} \circ \varphi_{1}\right)(p)\right\} & \text { if } p \leq p^{i \prime} \\
\min \left\{\left(\varphi_{2} \circ \varphi_{2}\right)(p),\left(\varphi_{2} \circ \varphi_{1}\right)(p)\right\} & \text { if } p \geq p^{i \prime}\end{cases} \\
& =\left\{\text { if } p^{i \prime} \geq p^{i}\left\{\begin{array}{c}
\left(\varphi_{1} \circ \varphi_{2}\right)(p) \text { if } p \leq p^{i} \\
\left(\varphi_{1} \circ \varphi_{1}\right)(p) \text { if } p^{i}<p<p^{i \prime} \\
\left(\varphi_{2} \circ \varphi_{1}\right)(p) \text { if } p \geq p^{i \prime} \\
\left(\varphi_{1} \circ \varphi_{2}\right)(p) \text { if } p \leq p^{i \prime} \\
\left(\varphi_{2} \circ \varphi_{2}\right)(p) \text { if } p^{i \prime}<p<p^{i} \\
\left(\varphi_{2} \circ \varphi_{1}\right)(p) \text { if } p \geq p^{i}
\end{array}\right.\right.
\end{aligned}
$$


Proof: To prove the second equality above, suppose that $p^{i \prime} \geq p^{i}$. Then for every price smaller than $p^{i}, p \leq p^{i}$, the definition of the intersecting price $p^{i}$ implies that $\varphi_{2}(p) \geq \varphi_{1}(p)$, and since $\left(\varphi_{1}\right)^{\prime}()<$.0 we have that $\left(\varphi_{1} \circ \varphi_{2}\right)(p) \leq$ $\left(\varphi_{1} \circ \varphi_{1}\right)(p)$. Now since $\varphi^{2}(p)=\min \left\{\left(\varphi_{1} \circ \varphi_{2}\right)(p),\left(\varphi_{1} \circ \varphi_{1}\right)(p)\right\}$ if $p \leq p^{i \prime}$ from the first equality, it follows that $\varphi^{2}(p)=\left(\varphi_{1} \circ \varphi_{2}\right)(p)$ for all prices $p \leq p^{i}$ which is the very first row behind the second equality in the case $p^{i \prime} \geq p^{i}$. For the second row, we have prices between both intersecting prices $p^{i}<p<p^{i \prime}$. By the same reasoning, $p \geq p^{i} \Longrightarrow \varphi_{2}(p) \leq \varphi_{1}(p)$ and using $\left(\varphi_{1}\right)^{\prime}()<$.0 we have that $\left(\varphi_{1} \circ \varphi_{2}\right)(p) \geq\left(\varphi_{1} \circ \varphi_{1}\right)(p)$. Now since $\varphi^{2}(p)=\min \left\{\left(\varphi_{1} \circ \varphi_{2}\right)(p),\left(\varphi_{1} \circ \varphi_{1}\right)(p)\right\}$ if $p \leq p^{i \prime}$ from the first equality, it follows that $\varphi^{2}(p)=\left(\varphi_{1} \circ \varphi_{1}\right)(p)$ for all prices $p^{i}<p<p^{i \prime}$. Finally, for the third row, prices satisfy $p \geq p^{i \prime}$ so that $\varphi^{2}(p)=\min \left\{\left(\varphi_{2} \circ \varphi_{2}\right)(p),\left(\varphi_{2} \circ \varphi_{1}\right)(p)\right\}$, but since $p^{i \prime} \geq p^{i} \Longrightarrow p \geq p^{i}$ and therefore $\varphi_{2}(p) \leq \varphi_{1}(p)$. By $\left(\varphi_{2}\right)^{\prime}()<$.0 we have that $\left(\varphi_{2} \circ \varphi_{2}\right)(p) \geq$ $\left(\varphi_{2} \circ \varphi_{1}\right)(p) \Longrightarrow \varphi^{2}(p)=\min \left\{\left(\varphi_{2} \circ \varphi_{2}\right)(p),\left(\varphi_{2} \circ \varphi_{1}\right)(p)\right\}=\left(\varphi_{2} \circ \varphi_{1}\right)(p)$, which is what we wanted to show. To prove the case $p^{i \prime} \leq p^{i}$ the same argument applies. Q.E.D.

Now, by defining $p_{\text {inf }}^{i}=\max \left\{\min \left\{p^{i \prime}, p^{i}\right\}, p_{1}\right\}$ and $p_{\text {sup }}^{i}=\min \left\{\max \left\{p^{i \prime}, p^{i}\right\}, p_{\infty}\right\}$, we can re-express in a more compact form $\varphi^{2}(p)$ as:

$\varphi^{2}(p)=\left\{\begin{array}{c}\left(\varphi_{1} \circ \varphi_{2}\right)(p) \text { if } p \leq p_{\mathrm{inf}}^{i} \\ \left(\varphi_{1} \circ \varphi_{1}\right)(p) \mathbf{1}_{\left\{p_{\mathrm{inf}}^{i}=p^{i}\right\}}+\left(\varphi_{2} \circ \varphi_{2}\right)(p) \mathbf{1}_{\left\{p_{\mathrm{inf}}^{i}=p^{i \prime}\right\}} \\ \left(\varphi_{2} \circ \varphi_{1}\right)(p) \text { if } p \geq p_{\mathrm{sup}}^{i}\end{array}\right.$ if $p \in\left(p_{\mathrm{inf}}^{i}, p_{\mathrm{sup}}^{i}\right)$

Where the symbol $\mathbf{1}_{\left\{p_{\mathrm{inf}}^{i}=p^{i}\right\}}$ denotes the standard indicator function, taking value 1 if $p_{\mathrm{inf}}^{i}=p^{i}$ and 0 otherwise.

Observations:

1) Since it can both happen that $p^{i \prime} \leq p^{i}$ and $p^{i \prime} \geq p^{i}$ so that $p^{i \prime}=p^{i}$, the definition of $\varphi^{2}(p)$ is correct and specializes to:

$$
\varphi^{2}(p)=\left(\varphi_{1} \circ \varphi_{2}\right)(p)=\left(\varphi_{2} \circ \varphi_{1}\right)(p), \forall p
$$

Proof: To see it, observe that if $p^{i \prime}=p^{i}$ from the definitions of $p_{\mathrm{inf}}^{i}$ and $p_{\text {sup }}^{i}$, we have that $p_{\text {inf }}^{i}=p_{\text {sup }}^{i}=p^{i}=p^{i \prime}$. Then:

$$
\begin{aligned}
& \varphi^{2}(p)=\left\{\begin{array}{c}
\left(\varphi_{1} \circ \varphi_{2}\right)(p) \text { if } p \leq p_{\mathrm{inf}}^{i}=p_{\mathrm{sup}}^{i} \\
\left(\varphi_{1} \circ \varphi_{1}\right)(p) \mathbf{1}_{\left\{p_{\mathrm{inf}}^{i}=p^{i}\right\}}+\left(\varphi_{2} \circ \varphi_{2}\right)(p) \mathbf{1}_{\left\{p_{\mathrm{inf}}^{i}=p^{i \prime}\right\}} \\
\left(\varphi_{2} \circ \varphi_{1}\right)(p) \text { if } p \geq p_{\mathrm{inf}}^{i}=p_{\mathrm{sup}}^{i}
\end{array} \text { if } p=\{\emptyset\}\right. \\
& =\left\{\begin{array}{l}
\left(\varphi_{1} \circ \varphi_{2}\right)(p) \text { if } p \leq p_{\text {inf }}^{i}=p_{\text {sup }}^{i} \\
\left(\varphi_{2} \circ \varphi_{1}\right)(p) \text { if } p \geq p_{\text {inf }}^{i}=p_{\text {sup }}^{i}
\end{array}\right.
\end{aligned}
$$

Also since $\bar{p} \in\left[p_{\text {inf }}^{i}, p_{\text {sup }}^{i}\right]=\left\{p_{\text {inf }}^{i}=p_{\text {sup }}^{i}\right\}$, from the proof above we must have that $\bar{p}=p_{\mathrm{inf}}^{i}=p_{\mathrm{sup}}^{i}=p^{i \prime}=p^{i}$. Recalling from appendix 1 that $\bar{p}=p^{i}$ whenever $C_{\Sigma}=\bar{C}_{\Sigma}$ and using the above explicit formulas for $\left(\varphi_{1} \circ \varphi_{2}\right)(p)$ and $\left(\varphi_{2} \circ \varphi_{1}\right)(p)$, observe that they have the same slope for all possible prices $p$ in 
their respective domains. Since they are both linear with the same slope, they will coincide provided that their constant terms coincide:

$$
\begin{aligned}
\frac{A_{\Sigma}}{B_{\Sigma}}+\frac{A_{2}}{B_{2}}\left(-\frac{C_{\Sigma}}{B_{\Sigma}}\right) & =\frac{A_{2}}{B_{2}}+\frac{A_{\Sigma}}{B_{\Sigma}}\left(-\frac{C_{\Sigma}}{B_{2}}\right) \Longleftrightarrow \\
& \Longleftrightarrow C_{\Sigma}=A_{2}\left[\frac{B_{1}}{A_{1}}-\frac{B_{2}}{A_{2}}\right] \equiv \bar{C}_{\Sigma}
\end{aligned}
$$

Stating that the constant terms in the definition of the functions $\left(\varphi_{1} \circ \varphi_{2}\right)(p)$ and $\left(\varphi_{2} \circ \varphi_{1}\right)(p)$ coincide whenever the aggregate cost parameter $C_{\Sigma}$ takes the value $\bar{C}_{\Sigma}$ compatible with $\bar{p}=p^{i}$. Therefore, $\varphi^{2}(p)=\left(\varphi_{1} \circ \varphi_{2}\right)(p)=$ $\left(\varphi_{2} \circ \varphi_{1}\right)(p), \forall p$ as we wanted to show. Q.E.D.

2) The reader must notice that the price domain of the second iterate of the cobweb function can exclude one of the limit prices $\left(p_{\text {inf }}^{i}, p_{\text {sup }}^{i}\right)$ defining the interval inside which the price equilibrium is determinate. To this purpose, suppose first that $p_{\mathrm{inf}}^{i}=p^{i}$ and $\varphi^{2}\left(p^{i}\right)<0$. Then since $\varphi^{2}($.) is (weakly) increasing, $\exists p_{1}^{\prime}: \forall \varepsilon>0, \varphi^{2}\left(p_{1}^{\prime}+\varepsilon\right)>0>\varphi^{2}\left(p^{i}\right)$. Then, we can enlarge the definition of $p_{\text {inf }}^{i}$ to $p_{\text {inf }}^{i}=\max \left\{\min \left\{p^{i \prime}, p^{i}\right\}, p_{1}^{\prime}\right\}$. Proceeding identically for $p_{\text {sup }}^{i}$ we can redefine it as $p_{\text {sup }}^{i}=\min \left\{\max \left\{p^{i \prime}, p^{i}\right\}, p_{\infty}\right\}$ where $p_{\infty}$ is the upper limit of the cobweb function price domain, i.e. $p_{\infty} \equiv \varphi_{1}^{-1}(0)=\frac{A_{\Sigma}}{C_{\Sigma}}$. If $\max \left\{p^{i \prime}, p^{i}\right\}=p^{i \prime}$ but $p^{i \prime}>p_{0}$, then we shall let $p_{\text {sup }}^{i}=p_{\infty}$ according to our enlarged definition. We will refer in the text to the enlarged definitions of $p_{\text {sup }}^{i}$ and $p_{\text {inf }}^{i}$ when appropriate.

\section{Appendix 3}

Properties of $\varphi^{2}($.$) as given by Guesnerie [11]:$

1) $\varphi^{2}($.$) is (weakly) increasing: since \partial \varphi^{2}(p)=\varphi^{\prime}[\varphi(p)] \varphi^{\prime}(p)$ and $\varphi^{\prime}()<$.0 , we must have $\partial \varphi^{2}(p)>0$ (a.e.).

2) $\varphi^{2}(\bar{p})=\bar{p}$ : trivial from $\varphi(\bar{p})=\bar{p}$ since $\varphi^{2}(\bar{p})=\varphi[\varphi(\bar{p})]=\varphi[\bar{p}]=\bar{p}$.

3) The success of eductive learning can be assessed entirely from $\varphi^{2}($.$) as$ long as there exists some CK (initial) information on prices (that starts the eductive game of guessing, i.e. $\left.p \leq p_{0}\right)$ and that $\lim _{\tau \rightarrow \infty}\left(\varphi^{2}\right)^{\tau}\left(p_{0}\right)=\bar{p}$.

\section{Appendix 4}

We completely characterize the learning dynamics of proposition 7 . The results are summarized in Table A4.1, and the definitions of the symbols immediately follow: 
Table A4.1: Summary of Results of Proposition 7

\begin{tabular}{|c|c|c|c|c|c|c|c|}
\hline$C_{\Sigma}$ & & & & & & & \\
\hline$>$ & {$\left[0, p_{0}\right]$} & {$\left[0, p_{0}\right]$} & {$\left[0, p_{0}\right]$} & {$\left[0, p_{0}\right]$} & {$\left[0, p_{0}\right]$} & {$\left[0, p_{0}\right]$} & 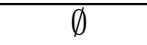 \\
\hline$=C_{\Sigma}^{1}$ & {$\left[0, p_{0}\right]$} & {$\left[0, p_{0}\right]$} & {$\left[0, p_{0}\right]$} & {$\left[0, p_{0}\right]$} & {$\left[0, p_{0}\right]$} & {$\left[0, p_{0}\right]$} & $\begin{array}{l}\emptyset \\
\emptyset \\
\emptyset\end{array}$ \\
\hline$\geq$ & $\{\bar{p}\}$ & $\left.\{\bar{p}\}\right|_{P}$ & $\left.\{\bar{p}\}\right|_{P}$ & $\left.\{\bar{p}\}\right|_{P}$ & $\left.\{\bar{p}\}\right|_{P}$ & $\begin{array}{l}\left.\{\bar{p}\}\right|_{P} \\
{\left[0, p_{0}\right]} \\
{\left[0, p_{0}\right]}\end{array}$ & $\emptyset$ \\
\hline$=C_{\Sigma}^{0}$ & $\{\bar{p}\}$ & $\left.\{\bar{p}\}\right|_{P}$ & $\left.\{\bar{p}\}\right|_{P}$ & $\left.\{\bar{p}\}\right|_{P}$ & $\begin{array}{c}\left.\{\bar{p}\}\right|_{P} \\
{\left[0, p_{0}\right]} \\
{\left[\bar{p}_{c 1}, \bar{p}_{c 2}\right]} \\
\end{array}$ & {$\left[\bar{p}_{c 1}, \bar{p}_{c 2}\right]$} & $\emptyset$ \\
\hline$>$ & $\{\bar{p}\}$ & $\{\bar{p}\}$ & $\{\bar{p}\}$ & $\begin{array}{c}\{\bar{p}\} \\
\{\bar{p}\} \\
{\left[\bar{p}_{c 1}, \bar{p}_{c 2}\right]}\end{array}$ & {$\left[\bar{p}_{c 1}, \bar{p}_{c 2}\right]$} & {$\left[\bar{p}_{c 1}, \bar{p}_{c 2}\right]$} & $\emptyset$ \\
\hline$=C_{\Sigma}^{2}$ & $\{\bar{p}\}$ & $\{\bar{p}\}$ & $\begin{array}{c}\{\bar{p}\} \\
\{\bar{p}\} \\
{\left[\bar{p}_{c 1}, \bar{p}_{c 2}\right] \mid}\end{array}$ & {$\left[\bar{p}_{c 1}, \bar{p}_{c 2}\right] \mid$} & {$\left[\bar{p}_{c 1}, \bar{p}_{c 2}\right] \mid$} & {$\left[\bar{p}_{c 1}, \bar{p}_{c 2}\right] \mid$} & $\emptyset$ \\
\hline$>$ & $\{\bar{p}\}$ & $\begin{array}{l}\{\bar{p}\} \\
\{\bar{p}\} \\
\{\bar{p}\} \\
\end{array}$ & $\{\bar{p}\}$ & $\{\bar{p}\}$ & $\{\bar{p}\}$ & $\{\bar{p}\}$ & $\emptyset$ \\
\hline 0 & $\begin{array}{l}\emptyset \\
\emptyset \\
\emptyset\end{array}$ & $\emptyset$ & $\emptyset$ & $\emptyset$ & $\emptyset$ & $\emptyset$ & $\emptyset$ \\
\hline $\left.\begin{array}{l}C_{\Sigma}>\bar{C}_{\Sigma} \\
C_{\Sigma}=\bar{C}_{\Sigma} \\
C_{\Sigma}<\bar{C}_{\Sigma}\end{array}\right)$ & 0 & $<$ & $=C_{\Sigma}^{2}$ & $<$ & $=C_{\Sigma}^{0}$ & $\leq$ & $C_{\Sigma}^{1}=\bar{C}_{\Sigma}$ \\
\hline
\end{tabular}

The contents, following the results of proposition 2, indicate the set of rationalizable-expectations equilibria, where the exogenous price restriction $p_{0}$ is embedded in the model (it is the maximum willingness to pay of the integrated economy demand):

-" $\left[0, p_{0}\right]$ " means that the set of rationalizable expectations equilibria usually contains the whole price domain $\left[0, p_{0}\right]$. As farmers learn nothing, $\bar{p}$ is not an SREE;

-" $\left[\bar{p}_{c 1}, \bar{p}_{c 2}\right]$ " means that the set of rationalizable prices is the whole segment $\left[\bar{p}_{c 1}, \bar{p}_{c 2}\right] \supset \bar{p}$, where $p_{c 2}=\varphi\left(p_{c 1}\right), \varphi^{2}\left(p_{c t}\right)=p_{c t}, t=1,2$ define cycles of order two of the cobweb function. For some parameterizations, the embedded price restriction $p_{0}$ can belong to the set $\left[\bar{p}_{c 1}, \bar{p}_{c 2}\right]$. Then, an exogenous price invertevention is called for restricting $p_{0}$ to be out of it: $p_{0} \notin\left[\bar{p}_{c 1}, \bar{p}_{c 2}\right]$, denoting such a requirement by " $\left[\bar{p}_{c 1}, \bar{p}_{c 2}\right] \mid$ ", meaning ' $\left[\bar{p}_{c 1}, \bar{p}_{c 2}\right]$ is the set of rationalizable prices conditional to that price restriction'.

-" $\{\bar{p}\}$ " means that the only rationalizable-expectations price equilibrium is the PFE $\bar{p}$, and $\bar{p}$ is an SREE.

-" $\left.\{\bar{p}\}\right|_{P}$ " means that the only rationalizable-expectations price equilibrium 
is the PFE $\bar{p}$ conditional to an exogenous price intervention restricting the natural one $p_{0}$ to be in the basin of attraction ${ }^{56}$ of $\bar{p}, P(\bar{p})=\left(\bar{p}_{c 1}, \bar{p}_{c 2}\right) \backslash\{\bar{p}\}$, and $\bar{p}$ is an 'SREE conditional to that price restriction'.

As the characterization of $\varphi^{2}($.$) depends on p^{i \prime}, p^{i}$ the learning dynamics will ultimately depend on whether $C_{\Sigma} \gtreqless \bar{C}_{\Sigma}$, since from the definitions of $p^{i \prime}, p^{i}$ and their properties we know that they depend on the value of the aggregate cost parameter $C_{\Sigma}$. In principle, $C_{\Sigma} \in \mathbb{R}_{++}$. We are going to divide the $C_{\Sigma^{-}}$ parameter space in four regions according to the following definitions of $C_{\Sigma}^{0}, C_{\Sigma}^{1}$ and $C_{\Sigma}^{2}$ satisfying:

$$
+\infty>C_{\Sigma}^{1} \geq C_{\Sigma}^{0}>C_{\Sigma}^{2}>0
$$

With $C_{\Sigma}^{1} \equiv B_{\Sigma}$ characterizing the limit value of the aggregate cost parameter above which the PFE price $\bar{p}$ becomes eductively unstable; $C_{\Sigma}^{0} \equiv B_{2}\left[1+\frac{A_{1}}{A_{2}}\right]$ satisfies $\lim _{\frac{A_{2}}{B_{2}} \rightarrow \frac{A_{1}}{B_{1}}} C_{\Sigma}^{1}=C_{\Sigma}^{0}$ so that the whole region $\left[C_{\Sigma}^{0}, C_{\Sigma}^{1}\right]$ collapses into that value $\left\{C_{\Sigma}^{0}\right\}$. Finally $C_{\Sigma}^{2} \equiv B_{2}$ characterizes the limit value of the aggregate cost parameter below which the PFE price $\bar{p}$ becomes eductively stable, i.e. if $C_{\Sigma}^{2}>C_{\Sigma}>0 \Longrightarrow \bar{p}$ is globally an SREE. Since the difference in the maximal willingnesses to pay is measured by the parameter $\bar{C}_{\Sigma}$, its range of variation will also be constrained to the regions for $C_{\Sigma}\left({ }^{57}\right)$. We allow the possibility that $0=\bar{C}_{\Sigma}$ because it corresponds to the case studied in proposition 6 .

\section{Proof of the results in Table A4.1.}

We are going to distinguish three broad cases, (those in brackets in the south-west corner of Table A4.1):

(*) Case $C_{\Sigma}>\bar{C}_{\Sigma}$ (We prove the results in the upper triangular matrix excluding the diagonal elements in the first bissectrix of the plane $\left.\left(\bar{C}_{\Sigma}, C_{\Sigma}\right)\right)$

a) If $C_{\Sigma}>\bar{C}_{\Sigma} \Longrightarrow p^{i}<p^{i \prime} \equiv \varphi_{1}^{-1}\left(p^{i}\right)$ by property 3 of $p^{i \prime}$. Then, by the definitions of $p_{\mathrm{inf}}^{i}, p_{\text {sup }}^{i}$ we have:

$$
\begin{aligned}
& p_{\text {inf }}^{i}=\max \left\{p^{i}, p_{1}^{\prime}\right\}: p_{1}^{\prime} \equiv\left(\varphi_{1} \circ \varphi_{1}\right)^{-1}(0)=p_{\infty}\left[1-\frac{B_{\Sigma}}{C_{\Sigma}}\right] \\
& p_{\text {sup }}^{i}=\min \left\{p_{1}^{i \prime}, p_{\infty}\right\}: p_{1}^{i \prime}=p_{\infty}-\frac{B_{\Sigma}}{C_{\Sigma}} p^{i}
\end{aligned}
$$

So that, since $\frac{B_{\Sigma}}{C_{\Sigma}}>0$ and $p^{i}>0$, we have $p_{1}^{i \prime}<p_{\infty}$ implying that $p_{\text {sup }}^{i}=p_{1}^{i \prime}$. On the other hand, the definition of $p_{1}^{\prime}$ implies that $p_{1}^{\prime}>0 \Longleftrightarrow 1>\frac{B_{\Sigma}}{C_{\Sigma}}$ since $p_{\infty}>0$ which is necessary for $p_{1}^{\prime}$ to be greater than $p^{i}>0$ although not sufficient. The sufficient condition is that $C_{\Sigma}>\bar{C}_{\Sigma}+B_{\Sigma} \Longrightarrow p_{\mathrm{inf}}^{i}=p_{1}^{\prime}$ and that the second iterate of the cobweb function is:

\footnotetext{
${ }^{56}$ The basin of attraction $P(\bar{p})$ of a given equilibrium price $\bar{p}$ is composed by the union of all the $p_{0} \neq \bar{p}$ s.t. $\lim _{\tau \longrightarrow+\infty} \varphi^{\tau}\left(p_{0}\right)=\bar{p}$.

${ }^{57}$ With the exception introduced by property 3 of the (second) intersecting price $p^{i \prime}$ according to which $0 \leq \bar{C}_{\Sigma}<C_{\Sigma}^{1}$. Details are in appendix 2 .
} 


$$
\varphi^{2}(p)= \begin{cases}\left(\varphi_{1} \circ \varphi_{1}\right)(p), & \text { if } p \leq p_{1}^{i \prime} \\ \left(\varphi_{2} \circ \varphi_{1}\right)(p), & \text { if } p \geq p_{1}^{i \prime}\end{cases}
$$

And by $p_{1}^{\prime}>0 \Longleftrightarrow 1>\frac{B_{\Sigma}}{C_{\Sigma}}$ we have that $\left(\varphi^{2}\right)^{\prime}(\bar{p})=\left(\varphi_{1} \circ \varphi_{1}\right)^{\prime}(\bar{p})=\left(\frac{C_{\Sigma}}{B_{\Sigma}}\right)^{2}>1$ and in consequence $\bar{p}$ is not strongly rational in $\left[p_{1}^{\prime}, p_{\infty}\right]$, corresponding to the upper north-west corner of Table A4.1 (noticing that $B_{\Sigma} \equiv C_{\Sigma}^{1}$ so that $C_{\Sigma}>$ $\bar{C}_{\Sigma}+C_{\Sigma}^{1}>C_{\Sigma}^{1}$ ) but also applies to the whole first row (because $\bar{C}_{\Sigma} \geq 0$,.we have that $C_{\Sigma}>\bar{C}_{\Sigma}+C_{\Sigma}^{1} \Longrightarrow C_{\Sigma}>C_{\Sigma}^{1}$ ). The set of rationalizable expectations equilibria is $\left[p_{1}^{\prime}, p_{\infty}\right] \subset\left[0, p_{0}\right]$, that we note $\left[0, p_{0}\right]$ in the table for simplicity. Q.E.D.

b) Now, considering the polar case, $\bar{C}_{\Sigma}<C_{\Sigma} \leq \bar{C}_{\Sigma}+C_{\Sigma}^{1} \Longrightarrow p_{\text {inf }}^{i}=p^{i}$ and the definition of the second iterate of the cobweb function becomes:

$$
\varphi^{2}(p)=\left\{\begin{array}{c}
\left(\varphi_{1} \circ \varphi_{2}\right)(p), \text { if } p \leq p^{i} \\
\left(\varphi_{1} \circ \varphi_{1}\right)(p), \text { if } p \in\left(p^{i}, p_{1}^{i \prime}\right) \\
\left(\varphi_{2} \circ \varphi_{1}\right)(p), \text { if } p \geq p_{1}^{i \prime}
\end{array}\right.
$$

Suppose then that $C_{\Sigma}=C_{\Sigma}^{1}$, implying no restriction on $\bar{C}_{\Sigma}$ by $C_{\Sigma} \leq \bar{C}_{\Sigma}+C_{\Sigma}^{1}$. Observe that $C_{\Sigma}=C_{\Sigma}^{1} \Longleftrightarrow 1=\frac{B_{\Sigma}}{C_{\Sigma}}$ and therefore $\left(\varphi^{2}\right)^{\prime}(\bar{p})=\left(\varphi_{1} \circ \varphi_{1}\right)^{\prime}(\bar{p})=$ $\left(\frac{C_{\Sigma}}{B_{\Sigma}}\right)^{2}=1, \forall p \in\left[p^{i}, p_{1}^{i \prime}\right] \supset \bar{p}$. Since $\left(\varphi_{1} \circ \varphi_{1}\right)(p)$ is a linear function of $p$ in $\left[p^{i}, p_{1}^{i \prime}\right]$, the whole segment is the set of rationalizable expectations equilibria. This segment is nonempty provided that $p_{1}^{i \prime}>p^{i} \Longleftrightarrow \frac{A_{2}}{B_{2}}<\frac{A_{1}}{B_{1}}\left[2+\frac{B_{1}}{B_{2}}\right] \Longleftrightarrow$ $\bar{C}_{\Sigma}<C_{\Sigma}^{1}$ which is always the case as showed above. Concerning the eductive stability of the segment of rationalizable expectations equilibria, observe that it depends on whether $\left(\varphi_{1} \circ \varphi_{2}\right)^{-1}(0) \equiv p_{1} \lesseqgtr 0$ and on $\left(\varphi_{1} \circ \varphi_{2}\right)^{\prime}(p)=\frac{C_{\Sigma}}{B_{\Sigma}} \frac{C_{\Sigma}}{B_{2}} \equiv$ $\frac{C_{\Sigma}}{C_{\Sigma}^{2}} \frac{C_{\Sigma}}{C_{\Sigma}^{1}}$. We have that:

$$
p_{1}=\frac{C_{\Sigma}^{2}}{C_{\Sigma}}\left(p_{0}-p_{\infty}\right) \underset{(\leq)}{>} 0 \Longleftrightarrow p_{0} \underset{(\leq)}{\gtrless} p_{\infty} \Longleftrightarrow C_{\Sigma} \underset{(\leq)}{\gtrless} C_{\Sigma}^{0}
$$

Since $C_{\Sigma}=C_{\Sigma}^{1}>C_{\Sigma}^{0}>C_{\Sigma}^{2}$ we must have $\left(\varphi_{1} \circ \varphi_{2}\right)^{-1}(0) \equiv p_{1}>0$ and $\left(\varphi_{1} \circ \varphi_{2}\right)^{\prime}(p)=\left.\frac{C_{\Sigma}}{C_{\Sigma}^{2}} \frac{C_{\Sigma}}{C_{\Sigma}^{1}}\right|_{C_{\Sigma}=C_{\Sigma}^{1}}>1$, for every price $p \in\left[p_{1}, p^{i}\right]$. But as well, by $\left(\varphi_{1} \circ \varphi_{2}\right)^{\prime}(p)=\left(\varphi_{2} \circ \varphi_{1}\right)^{\prime}(p)>1, \forall p \in\left[p_{1}^{i \prime}, p_{\infty}\right]$. In consequence $\bar{p}$ is not strongly rational in $\left[p_{1}, p_{\infty}\right]$, the set of rationalizable expectations equilibria is a connected segment $\left[p^{i}, p_{1}^{i \prime}\right] \supset \bar{p}$ that we note " $\left[0, p_{0}\right]$ " for simplicity. It also applies to the whole second row because we have not imposed any restriction on $\bar{C}_{\Sigma}$ except that $\bar{C}_{\Sigma}<C_{\Sigma}$,. which is always satisfied. Q.E.D.

Now suppose that $C_{\Sigma}<C_{\Sigma}^{1}$. We will distinguish two cases:

b.1.) $C_{\Sigma}^{0} \leq C_{\Sigma}<C_{\Sigma}^{1} \Longrightarrow\left(\varphi^{2}\right)^{\prime}(\bar{p})=\left(\varphi_{1} \circ \varphi_{1}\right)^{\prime}(\bar{p})=\left(\frac{C_{\Sigma}}{C_{\Sigma}^{1}}\right)^{2}<1, \forall p \in$ $\left[p^{i}, p_{1}^{i \prime}\right] \supset \bar{p}$, by the second inequality. This shows that $\bar{p}$ will be strongly 
rational on its basin of attraction, which will be non-empty since it will contain at least $\forall p \in\left[p^{i}, p_{1}^{i \prime}\right] \supset \bar{p}$.

Lemma b.1.): $\left(\varphi_{1} \circ \varphi_{1}\right)\left(p^{i}\right)>p^{i}$.

Proof: This is equivalent to

$$
\begin{aligned}
\frac{A_{\Sigma}}{B_{\Sigma}}\left[1-\frac{C_{\Sigma}}{C_{\Sigma}^{1}}\right]+\frac{C_{\Sigma}}{C_{\Sigma}^{1}} \frac{C_{\Sigma}^{2}}{C_{\Sigma}^{1}}\left[\frac{A_{2}}{B_{2}}-\frac{A_{1}}{B_{1}}\right] & >\frac{C_{\Sigma}^{2}}{C_{\Sigma}}\left[\frac{A_{2}}{B_{2}}-\frac{A_{1}}{B_{1}}\right] \Longleftrightarrow \\
\frac{A_{\Sigma}}{B_{\Sigma}} & >\frac{A_{1}}{B_{1}} \frac{\bar{C}_{\Sigma}}{C_{\Sigma}}
\end{aligned}
$$

But since $C_{\Sigma}>\bar{C}_{\Sigma}$ we have that $\frac{A_{1}}{B_{1}}>\frac{A_{1}}{B_{1}} \frac{\bar{C}_{\Sigma}}{C_{\Sigma}}$ whereas $\frac{A_{\Sigma}}{B_{\Sigma}}>\frac{A_{1}}{B_{1}}$ so that the above inequality is always true and $\left(\varphi_{1} \circ \varphi_{1}\right)\left(p^{i}\right)>p^{i}$ which is what we wanted to show. Q.E.D.

By definition of $p^{i}$ and by the result just proved, we have $\left(\varphi_{1} \circ \varphi_{1}\right)\left(p^{i}\right)=$ $\left(\varphi_{1} \circ \varphi_{2}\right)\left(p^{i}\right)>p^{i}$. By the first weak inequality of case b.1.), the definition of $p_{1}$ implies that $p_{1} \geq 0 \Longleftrightarrow\left(\varphi_{1} \circ \varphi_{2}\right)\left(p_{1}\right)=0$. By Weierstrass' theorem, $\exists \bar{p}_{c 1} \in$ $\left[p_{1}, p^{i}\right]:\left(\varphi_{1} \circ \varphi_{2}\right)\left(\bar{p}_{c 1}\right)=\bar{p}_{c 1}$. And since $\varphi^{2}($.$) is C^{1}$ in the domain $\left[p_{1}, p^{i}\right]$, by the mean value theorem, we must have $\left(\varphi_{1} \circ \varphi_{2}\right)^{\prime}\left(\bar{p}_{c 1}\right)=\frac{\left(\varphi_{1} \circ \varphi_{2}\right)\left(p^{i}\right)-\left(\varphi_{1} \circ \varphi_{2}\right)\left(p_{1}\right)}{p^{i}-p_{1}}>$ 1.

Replicating the same reasoning on the price domain $\left[p_{1}^{i \prime}, p_{\infty}\right]$ of $\varphi^{2}($.$) , we$ have that $\left(\varphi_{1} \circ \varphi_{1}\right)\left(p_{1}^{i \prime}\right)=\left(\varphi_{2} \circ \varphi_{1}\right)\left(p_{1}^{i \prime}\right)<p_{1}^{i \prime}$ where the inequality follows from the fact that by property 5 of $p^{i \prime}\left[p^{i}, p_{1}^{i \prime}\right] \supset \bar{p} \Longrightarrow p_{1}^{i \prime} \leq \bar{p}$ and by property 2 of $\varphi^{2}($.$) we have \bar{p}=\varphi^{2}(\bar{p})$, so that since $\left(\varphi_{1} \circ \varphi_{1}\right)^{\prime}(\bar{p})<1$ and $\left(\varphi^{2}\right)^{\prime}($.$) is$ of constant slope in $\left[p^{i}, p_{1}^{i \prime}\right]$, the inequality must be true. We also have that $\left(\varphi_{2} \circ \varphi_{1}\right)\left(p_{\infty}\right)>p_{\infty}$, a fact that can be observed by direct computation since equivalent to

$$
\frac{A_{2}}{C_{\Sigma}^{2}}-\frac{A_{\Sigma}}{C_{\Sigma}^{1}} \frac{C_{\Sigma}}{C_{\Sigma}^{2}}+\frac{C_{\Sigma}}{C_{\Sigma}^{1}} \frac{C_{\Sigma}}{C_{\Sigma}^{2}} \frac{A_{\Sigma}}{C_{\Sigma}}>\frac{A_{\Sigma}}{C_{\Sigma}} \Longleftrightarrow C_{\Sigma}>C_{\Sigma}^{0}
$$

Therefore by Weierstrass' theorem, $\exists \bar{p}_{c 2} \in\left[p_{1}^{i \prime}, p_{\infty}\right]:\left(\varphi_{2} \circ \varphi_{1}\right)\left(\bar{p}_{c 2}\right)=\bar{p}_{c 2}$. And since $\varphi^{2}($.$) is C^{1}$ in the domain $\left[p_{1}^{i \prime}, p_{\infty}\right]$, by the mean value theorem, we must have $\left(\varphi_{2} \circ \varphi_{1}\right)^{\prime}\left(\bar{p}_{c 2}\right)=\frac{\left(\varphi_{2} \circ \varphi_{1}\right)\left(p_{1}^{i \prime}\right)-\left(\varphi_{2} \circ \varphi_{1}\right)\left(p_{\infty}\right)}{p_{1}^{\nu^{\prime}}-p_{\infty}}>1$. Finally, notice that $\varphi_{1}\left(\bar{p}_{c 2}\right)=\bar{p}_{c 1}$ and that $\varphi_{2}\left(\bar{p}_{c 1}\right)=\bar{p}_{c 2}$ so that $\left\{\bar{p}_{c 1}, \bar{p}_{c 2}\right\}$ form a cycle of period two.

To summarize the results of case b.1.): $\left\{\bar{p}_{c 1}, \bar{p}_{c 2}\right\}$ constitute respectively the lower and upper bounds of the connected segment of the rationalizable expectations equilibria $\left(\bar{p}_{c 1}, \bar{p}_{c 2}\right) \supset \bar{p}$ defining the basin of attraction of $\bar{p}, P(\bar{p})$. When restricted to its basin of attraction, $\bar{p}$ is an "SREE subject to that restriction", i.e. $\forall p_{0} \in P(\bar{p})$, that we note " $\left.\{\bar{p}\}\right|_{P}$ " since it can happen, as in the text, that $p_{0} \notin P(\bar{p})$. This corresponds to the third and fourth rows of the upper triangular matrix in Table A4.1, excluding the first bissectrix elements and the first colum ones. The first column ones correspond to the case b.2.) because when $\bar{C}_{\Sigma}=0$, $C_{\Sigma}^{0}=C_{\Sigma}^{1}$ and case b.1.) collapses to case b.2.). Q.E.D. 
b.2.) $C_{\Sigma}<C_{\Sigma}^{0} \leq C_{\Sigma}^{1}$. By the first inequality and the definitions of $p_{1}, p_{\infty}$ we have that $p_{1}<0 \Longrightarrow\left(\varphi_{1} \circ \varphi_{2}\right)(0)>0$ and that $\left(\varphi_{2} \circ \varphi_{1}\right)\left(p_{\infty}\right)<p_{\infty}$ by the converse argument used in b.1.). It is still the case that $\left(\varphi_{1} \circ \varphi_{1}\right)^{\prime}(p)<1$ for all $p \in\left[p^{i}, p_{1}^{i \prime}\right] \supset \bar{p}$. Now, even if $\left(\varphi_{1} \circ \varphi_{2}\right)^{\prime}(p)=\left(\varphi_{2} \circ \varphi_{1}\right)^{\prime}(p) \gtrless 1$ in their respective price domains $\left[0, p^{i}\right]$ and $\left[p_{1}^{i \prime}, p_{\infty}\right], \bar{p}$ is the unique rationalizable expectations equilibrium and it is strongly rational in $\left[0, p_{\infty}\right]$ (globally). This corresponds to the results in the first column of table A4.1 $\left(\bar{C}_{\Sigma}=0\right)$ and to the fifth, sixth and seventh rows of the upper triangular matrix with respect to the first bissectrix, excluding the elements of the diagonal. Q.E.D.

This completes the proof of the results corresponding to case $C_{\Sigma}>\bar{C}_{\Sigma}$. Q.E.D.

(**) Case $C_{\Sigma}<\bar{C}_{\Sigma}$ (We prove the results in the lower triangular matrix excluding the diagonal elements in the first bissectrix of the plane $\left(\bar{C}_{\Sigma}, C_{\Sigma}\right)$ defined by Table A4.1)

a) If $C_{\Sigma}<\bar{C}_{\Sigma} \Longrightarrow p^{i}>p^{i \prime} \equiv \varphi_{2}^{-1}\left(p^{i}\right)$ by property 3 of $p^{i \prime}$. Then, by the definitions of $p_{\mathrm{inf}}^{i}, p_{\mathrm{sup}}^{i}$ we have:

$$
\begin{aligned}
& p_{\mathrm{inf}}^{i}=\max \left\{p_{2}^{i \prime}, p_{2}^{\prime}, 0\right\}: p_{2}^{i \prime}=\frac{A_{2}}{C_{\Sigma}}-\frac{B_{2}}{C_{\Sigma}} p^{i} \\
& p_{\text {sup }}^{i}=\min \left\{p^{i}, p_{\infty}\right\}: p^{i}=p_{\infty}-\frac{A_{1}}{C_{\Sigma}}\left[1+\frac{B_{2}}{B_{1}}\right]<p_{\infty} \Longrightarrow p_{\text {sup }}^{i}=p^{i}
\end{aligned}
$$

Where $p_{2}^{i \prime}=\frac{A_{2}}{C_{\Sigma}}-\frac{B_{2}}{C_{\Sigma}} p^{i}=p_{2}^{\prime}+\frac{A_{1}}{B_{1}}\left(\frac{B_{2}}{C_{\Sigma}}\right)^{2}>p_{2}^{\prime}$ and $p_{2}^{\prime} \equiv\left(\varphi_{2} \circ \varphi_{2}\right)^{-1}(0)=$ $\frac{A_{2}}{C_{\Sigma}}\left[1-\frac{B_{2}}{C_{\Sigma}}\right]$ implying that $p_{\text {inf }}^{i}=\max \left\{p_{2}^{i \prime}, 0\right\}$. From the definition of $p_{2}^{i \prime}$ we have $p_{2}^{i \prime} \leq 0 \Longleftrightarrow C_{\Sigma} \leq B_{2}\left[1-\frac{A_{1}}{B_{1}} \frac{B_{2}}{A_{2}}\right]<B_{2}$ by $\frac{A_{1}}{B_{1}} \leq \frac{A_{2}}{B_{2}}$, and by the definition of $p_{\mathrm{inf}}^{i}$ we have that $p_{\mathrm{inf}}^{i}=\max \left\{p_{2}^{i \prime}, 0\right\}=0$ which on its turn implies that $\left(\varphi_{2} \circ \varphi_{2}\right)(0)>0$, by $C_{\Sigma}<B_{2} \Longrightarrow p_{2}^{\prime}<0$, and the second iterate of the cobweb function becomes:

$$
\varphi^{2}(p)= \begin{cases}\left(\varphi_{2} \circ \varphi_{2}\right)(p), & \text { if } p<p^{i} \\ \left(\varphi_{2} \circ \varphi_{1}\right)(p), & \text { if } p \geq p^{i}\end{cases}
$$

Now, since $C_{\Sigma}<B_{2} \equiv C_{\Sigma}^{2}$, we have that $\left(\varphi_{2} \circ \varphi_{2}\right)^{\prime}(p)=\left(\frac{C_{\Sigma}}{C_{\Sigma}^{2}}\right)^{2}<1, \forall p<p^{i}$ and by property 5 of $p^{i \prime}$ we know that $\bar{p} \in\left[p^{i \prime}, p^{i}\right]$ whenever $C_{\Sigma}<\bar{C}_{\Sigma}$, letting $p^{i \prime}=0$. In consequence $\left(\varphi_{2} \circ \varphi_{2}\right)^{\prime}(\bar{p})<1$ so that $\bar{p}$ is eductively stable in $\left[0, p^{i}\right)$. That it is unique follows from the linearity (and therefore continuity) of $\varphi^{2}($.$) in the price domain \left[0, p^{i}\right)$, toghether with the facts $\left(\varphi_{2} \circ \varphi_{2}\right)(0)>0$ and $\left(\varphi_{2} \circ \varphi_{1}\right)\left(p^{i}\right)=\left(\varphi_{2} \circ \varphi_{2}\right)\left(p^{i}\right)<p^{i}$ that allow us to apply Weierstrass' theorem to $\varphi^{2}($.$) . To prove that \left(\varphi_{2} \circ \varphi_{2}\right)\left(p^{i}\right)<p^{i}$ we can use the explicit expression for 
$p^{i}=\frac{A_{2}}{C_{\Sigma}}-\frac{A_{1}}{B_{1}} \frac{B_{2}}{C_{\Sigma}}$ and operating we find:

$$
\begin{aligned}
\frac{A_{2}}{B_{2}}\left[1-\frac{C_{\Sigma}}{B_{2}}\right]+\left(\frac{C_{\Sigma}}{B_{2}}\right)^{2} p^{i} & <p^{i} \Longleftrightarrow \\
\frac{A_{2}}{B_{2}}-\frac{A_{1}}{B_{1}} \frac{C_{\Sigma}}{B_{2}} & <\frac{A_{2}}{C_{\Sigma}}-\frac{A_{1}}{B_{1}} \frac{B_{2}}{C_{\Sigma}} \Longleftrightarrow \\
C_{\Sigma} & <B_{2} \frac{A_{2}}{B_{2}} \frac{B_{1}}{A_{1}}\left[1-\frac{A_{1}}{B_{1}} \frac{B_{2}}{A_{2}}\right]
\end{aligned}
$$

Which, noticing that $B_{2}\left[1-\frac{A_{1}}{B_{1}} \frac{B_{2}}{A_{2}}\right] \leq B_{2} \frac{A_{2}}{B_{2}} \frac{B_{1}}{A_{1}}\left[1-\frac{A_{1}}{B_{1}} \frac{B_{2}}{A_{2}}\right]$ because $\frac{A_{2}}{B_{2}} \frac{B_{1}}{A_{1}} \geq 1$, and that we are considering values of the aggregate cost such that $C_{\Sigma} \leq$ $B_{2}\left[1-\frac{A_{1}}{B_{1}} \frac{B_{2}}{A_{2}}\right]$, it is always the case. Finally, $\forall p \geq p^{i}$ we have that $\left(\varphi_{2} \circ \varphi_{1}\right)^{\prime}(p)=$ $\left(\frac{C_{\Sigma}}{C_{\Sigma}^{2}} \frac{C_{\Sigma}}{C_{\Sigma}^{1}}\right)<1$, by $C_{\Sigma}^{1}>C_{\Sigma}^{2}>C_{\Sigma}$. Therefore, there exists a unique rationalizable expectations equilibrium price $\bar{p}$ which is an SREE in $\left[0, p_{\infty}\right]$ (globally).

If however $B_{2}\left[1-\frac{A_{1}}{B_{1}} \frac{B_{2}}{A_{2}}\right]<C_{\Sigma}<B_{2}$ then $p_{2}^{i \prime}>0 \Longrightarrow p_{\mathrm{inf}}^{i}=\max \left\{p_{2}^{i \prime}, 0\right\}=$ $p_{2}^{i \prime}$ and the second iterate of the cobweb function becomes:

$$
\varphi^{2}(p)=\left\{\begin{array}{c}
\left(\varphi_{1} \circ \varphi_{2}\right)(p), \text { if } p \leq p_{2}^{i \prime} \\
\left(\varphi_{2} \circ \varphi_{2}\right)(p), \text { if } p \in\left(p_{2}^{i \prime}, p^{i}\right) \\
\left(\varphi_{2} \circ \varphi_{1}\right)(p), \text { if } p \geq p^{i}
\end{array}\right.
$$

Since what was important for the existence and eductive stability of the equilibrium was that $C_{\Sigma}<B_{2}$ and by $\left(\varphi_{2} \circ \varphi_{1}\right)^{\prime}(p)=\left(\varphi_{1} \circ \varphi_{2}\right)^{\prime}(p)<1$, the same conclusions follow for this enlarged definition of $\varphi^{2}($.$) . This case corresponds to$ the bottom row of the lower triangular matrix in table A4.1, excluding the first bissectrix diagonal terms.Q.E.D.

b) Suppose now that $\bar{C}_{\Sigma}>C_{\Sigma}=B_{2}$. Then from the above definition of $p_{2}^{\prime}$, $p_{2}^{\prime}=0$ and as well, $\left(\varphi_{2} \circ \varphi_{2}\right)^{\prime}(p)=1$, for all $p \in\left(p_{2}^{i \prime}, p^{i}\right)$ implying that all the prices in this open interval will be rationalizable expectations prices. Whether they are eductively stable or not will depend on the slope of $\left(\varphi_{2} \circ \varphi_{1}\right)^{\prime}(p)=$ $\left(\varphi_{1} \circ \varphi_{2}\right)^{\prime}(p)$ which is smaller than one because $C_{\Sigma}^{1}>C_{\Sigma}^{2}=C_{\Sigma}$. Therefore, the set of rationalizable expectations prices is the segment $\left[p_{2}^{i \prime}, p^{i}\right] \supset \bar{p}$, that we note " $\left[\bar{p}_{c 1}, \bar{p}_{c 2}\right] \mid$ " for simplicity, and because $p_{0}$ must be taken outside it. Observe that $\forall p \in\left[p_{2}^{i \prime}, p^{i}\right] \backslash\{\bar{p}\}$ is an eductive cycle of period two. Since the reasoning is independent of the value of $\bar{C}_{\Sigma}$ as long as $\bar{C}_{\Sigma}>C_{\Sigma}$, the same is true for the whole before last bottom row of the lower triangular matrix excepting the elements in the firt bissectrix. Q.E.D.

c) The case $\bar{C}_{\Sigma}>C_{\Sigma}^{0} \geq C_{\Sigma}$ corresponds to the second and third rows of the lower triangular matrix of Table A4.1. Recalling that from the definition of $p_{1}$ from case $(*)$ we have,

$$
p_{1}=\frac{C_{\Sigma}^{2}}{C_{\Sigma}}\left(p_{0}-p_{\infty}\right) \underset{(\leq)}{\gtrless} 0 \Longleftrightarrow p_{0} \underset{(\leq)}{\gtrless} p_{\infty} \Longleftrightarrow C_{\Sigma} \underset{(\leq)}{\gtrless} C_{\Sigma}^{0}
$$


and that $\left(\varphi_{2} \circ \varphi_{2}\right)^{\prime}(p)=\left(\frac{C_{\Sigma}}{C_{\Sigma}^{2}}\right)^{2}>1$, for all $p \in\left(p_{2}^{i \prime}, p^{i}\right) \supset \bar{p}$. Therefore $\bar{p}$ will not be eductively stable in $\left(p_{2}^{i \prime}, p^{i}\right)$. Now since $\varphi^{2}(\bar{p})=\left(\varphi_{2} \circ \varphi_{2}\right)(\bar{p})=\bar{p}$ by property 2 of the second iterate of the cobweb function, and by property 5 of $p^{i \prime}, \bar{p} \in\left(p_{2}^{i \prime}, p^{i}\right)$ the linearity of $\varphi^{2}($.$) in prices implies that \left(\varphi_{1} \circ \varphi_{2}\right)\left(p_{2}^{i \prime}\right)=$ $\left(\varphi_{2} \circ \varphi_{2}\right)\left(p_{2}^{i \prime}\right)<\left(\varphi_{2} \circ \varphi_{2}\right)(\bar{p})=\bar{p}$ which on its turn implies that $\left(\varphi_{1} \circ \varphi_{2}\right)\left(p_{2}^{i \prime}\right)<$ $p_{2}^{i \prime}$. Now the definition of $p_{1}$ states that for the case under consideration, $p_{1} \leq$ $0 \Longrightarrow\left(\varphi_{1} \circ \varphi_{2}\right)(0) \geq 0$. By property 1 of $\varphi^{2}($.$) , we have that p_{2}^{i \prime}>0 \Longrightarrow$ $\left(\varphi_{1} \circ \varphi_{2}\right)\left(p_{2}^{i \prime}\right)>\left(\varphi_{1} \circ \varphi_{2}\right)(0) \geq 0$. Since $\varphi^{2}($.$) is linear, it is continuous and C^{1}$ in the domain $\left[0, p_{2}^{i \prime}\right]$, and we can apply the mean value theorem for $p \in\left(0, p_{2}^{i \prime}\right)$, $\left(\varphi_{1} \circ \varphi_{2}\right)^{\prime}(p)=\frac{\left(\varphi_{1} \circ \varphi_{2}\right)\left(p_{2}^{i \prime}\right)-\left(\varphi_{1} \circ \varphi_{2}\right)(0)}{p_{2}^{i \prime}}<\frac{\left(\varphi_{1} \circ \varphi_{2}\right)\left(p_{2}^{i \prime}\right)}{p_{2}^{i \prime}}<1$ by the inequality $\left(\varphi_{1} \circ \varphi_{2}\right)\left(p_{2}^{i \prime}\right)<p_{2}^{i \prime}$. By Weierstrass' theorem, we have that $\exists \bar{p}_{c 1} \in\left[0, p_{2}^{i \prime}\right]$ : $\left(\varphi_{1} \circ \varphi_{2}\right)\left(\bar{p}_{c 1}\right)=\bar{p}_{c 1}$, and by the mean value theorem, $\left(\varphi_{1} \circ \varphi_{2}\right)^{\prime}\left(\bar{p}_{c 1}\right)<1$. By observing that $\left(\varphi_{2} \circ \varphi_{1}\right)\left(p^{i}\right)=\left(\varphi_{2} \circ \varphi_{2}\right)\left(p^{i}\right)>\left(\varphi_{2} \circ \varphi_{2}\right)(\bar{p})=\bar{p}$ and therefore $\left(\varphi_{2} \circ \varphi_{1}\right)\left(p^{i}\right)>p^{i}$ by the parallel reasoning, but that now $\left(\varphi_{2} \circ \varphi_{1}\right)\left(p_{\infty}\right) \leq p_{\infty}$, and using exactly the same argument, we can conclude that $\exists \bar{p}_{c 2} \in\left[p^{i}, p_{\infty}\right]$ : $\left(\varphi_{2} \circ \varphi_{1}\right)\left(\bar{p}_{c 2}\right)=\bar{p}_{c 2}$, and by the mean value theorem, $\left(\varphi_{2} \circ \varphi_{1}\right)^{\prime}\left(\bar{p}_{c 2}\right)<1$. That $\left(\varphi_{2} \circ \varphi_{1}\right)\left(p_{\infty}\right) \leq p_{\infty}$ was shown to be the case whenever $C_{\Sigma}^{0} \geq C_{\Sigma}$ in case $\left(^{*}\right)$ part b.1.). Therefore we can conclude that the PFE price is not an SREE. $\left(\bar{p}_{c 1}, \bar{p}_{c 2}\right)$ is the interval of rationalizable prices provided that $p_{0} \notin\left(\bar{p}_{c 1}, \bar{p}_{c 2}\right)$, that we note " $\left[\bar{p}_{c 1}, \bar{p}_{c 2}\right]$ " for simplicity. The interval is bounded by the twoperiod cycle of the cobweb function: $\varphi_{1}\left(\bar{p}_{c 2}\right)=\bar{p}_{c 1}$ and $\varphi_{2}\left(\bar{p}_{c 1}\right)=\bar{p}_{c 2}$. Whenever $C_{\Sigma} \rightarrow C_{\Sigma}^{0} \equiv B_{2}\left[1+\frac{A_{1}}{A_{2}}\right]$, we have that $\bar{p}_{c 1} \rightarrow 0$ and $\bar{p}_{c 2} \rightarrow p_{\infty}$. This completes the study of the results in the second and third rows of the lower diagonal matrix, excluding the elements in the first bissectrix.Q.E.D.

d) The case $C_{\Sigma}^{1}>\bar{C}_{\Sigma}>C_{\Sigma}>C_{\Sigma}^{0}$ corresponds to the first row of the lower triangular matrix, below the first bissectrix of Table A4.1. Relative to case c) above, the only thing that changes is that since $C_{\Sigma}>C_{\Sigma}^{0} \Longrightarrow p_{1}>0 \Longleftrightarrow$ $\left(\varphi_{1} \circ \varphi_{2}\right)(0)<0$ but as well, $\left(\varphi_{2} \circ \varphi_{1}\right)\left(p_{\infty}\right)>p_{\infty}$ and in consequence, there will be no intersection with the first bissectrix other than $\bar{p}$. Therefore, $\bar{p}$ is not an SREE in $\left[p_{1}, p_{\infty}\right] \subset\left[0 . p_{0}\right]$, and the set of rationalizable prices is noted to be $"\left[0 . p_{0}\right] "$ for simplicity. Q.E.D.

This completes the study of the case $(* *) \bar{C}_{\Sigma}>C_{\Sigma}$. Q.E.D.

Finally the results included in the diagonal terms of the first bissectrix in Table A4.1, corresponding to $\bar{C}_{\Sigma}=C_{\Sigma}$, are easily proven recognizing that in such a case $p^{i}=p^{i \prime}=\bar{p}$ and that the second iterate of the cobweb function is a linear function on its price domain equal to

$$
\varphi^{2}(p)=\left(\varphi_{1} \circ \varphi_{2}\right)(p)=\left(\varphi_{2} \circ \varphi_{1}\right)(p), \forall p
$$

A fact proved in the derivation of the second iterate of the cobweb function, observation 1. Everything will here depend on whether $C_{\Sigma} \lesseqgtr C_{\Sigma}^{0}$ observing that 
the perfect foresight equilibrium price $\bar{p}$ will always be the unique rationalizableexpectations equilibrium (SREE in $\left[0, p_{\infty}\right]$ whenever $C_{\Sigma}<C_{\Sigma}^{0}$, and not an SREE in $\left[p_{1}, p_{\infty}\right]$ whenever $\left.C_{\Sigma}>C_{\Sigma}^{0}\right)$. When $C_{\Sigma}=C_{\Sigma}^{0}$, a continuum of rationalizableexpectations equilibria exists, composed by the segment $\left[0, p_{\infty}\right]$, and every price in the segment will be a period-two cycle of the cobweb function with the exception of $\bar{p}$. Q.E.D.

This completes the proof of the results in Table A4.1.

\section{Appendix 5}

Proof of Proposition 8

Since $\exists n, n^{\prime} \in \mathbf{N}: \frac{A_{n}}{B_{n}} \neq \frac{A_{n^{\prime}}}{B_{n^{\prime}}}$, assume that there exists a region the consumers of which will not be able to afford the consumption of the crop at the prevailing PFE price $\bar{p}$, we have that:

$$
\begin{aligned}
\sum_{n: \bar{p} \leq p_{0}^{n}} D_{n}^{\prime}(\bar{p}) & \geq \sum_{n} D_{n}^{\prime}(\bar{p}) \Longrightarrow \\
\frac{\sum_{n} D_{n}^{\prime}(\bar{p})}{\sum_{n: \bar{p} \leq p_{0}^{n}} D_{n}^{\prime}(\bar{p})}\left[\sum_{n} \alpha_{n} \varphi_{n}^{\prime}\left(\bar{p}_{n}\right)\right] & \leq \frac{\sum_{n} S_{n}^{\prime}(\bar{p})}{\sum_{n} D_{n}^{\prime}(\bar{p})}=\sum_{n} \alpha_{n} \varphi_{n}^{\prime}\left(\bar{p}_{n}\right)
\end{aligned}
$$

Taking absolute values on both sides:

$$
\left|\varphi^{\prime}(\bar{p})\right| \geq\left|\sum_{n} \alpha_{n} \varphi_{n}^{\prime}\left(\bar{p}_{n}\right)\right|
$$

So that when differences in the maximal willingness to pay for the crop exist (LHS), the PFE price is 'more unstable' than when they do not exist (RHSproposition 6 ). But we can measure by how much, since:

$$
\left|\varphi^{\prime}(\bar{p})\right|<1 \Longleftrightarrow\left|\sum_{n} \alpha_{n} \varphi_{n}^{\prime}\left(\bar{p}_{n}\right)\right|<\frac{\sum_{n: \bar{p} \leq p_{0}^{n}} D_{n}^{\prime}(\bar{p})}{\sum_{n} D_{n}^{\prime}(\bar{p})} \equiv \frac{1}{\varkappa_{\left\{n: \bar{p} \leq p_{0}^{n}\right\}}}
$$

With $\varkappa_{\left\{n: \bar{p} \leq p_{0}^{n}\right\}} \geq 1$, taking value 1 when the integration equilibrium price $\bar{p}$ is low enough so that the consumers of all the integrating regions can afford to pay it (the situation in proposition 6): i.e. $\sum_{n: \bar{p} \leq p_{0}^{n}} D_{n}^{\prime}(\bar{p})=\sum_{n} D_{n}^{\prime}(\bar{p})$. Then the conditions of proposition 6 are strengthened to:

$$
\varkappa_{\left\{n: \bar{p} \leq p_{0}^{n}\right\}} \min _{n}\left|\varphi_{n}^{\prime}\left(\bar{p}_{n}\right)\right| \leq\left|\varphi^{\prime}(\bar{p})\right| \leq \varkappa_{\left\{n: \bar{p} \leq p_{0}^{n}\right\}} \max _{n}\left|\varphi_{n}^{\prime}\left(\bar{p}_{n}\right)\right|
$$

Meaning that even if all autarkic price equilibria are expectationally stable, so that $\max _{n}\left|\varphi_{n}^{\prime}\left(\bar{p}_{n}\right)\right|<1$, the PFE price $\bar{p}$ can fail to be so whenever:

$$
\varkappa_{\left\{n: \bar{p} \leq p_{0}^{n}\right\}}>\frac{1}{\max _{n}\left|\varphi_{n}^{\prime}\left(\bar{p}_{n}\right)\right|} \Longrightarrow\left|\varphi^{\prime}(\bar{p})\right|>\max _{n}\left|\varphi_{n}^{\prime}\left(\bar{p}_{n}\right)\right|
$$


i.e. whenever there are sufficient economies the consumers of which cannot afford to pay the international price for the crop. The smaller the set of the economies in which consumers demand the crop at the international price $\left\{n: \bar{p} \leq p_{0}^{n}\right\}$, the smaller the elasticity of the integration aggregate demand, the larger the value of $\varkappa_{\left\{n: \bar{p} \leq p_{0}^{n}\right\}}$ above one, and the more likely becomes the above inequality. Q.E.D.

\section{Proof of Proposition 9}

The regional integration equilibrium price $\bar{p}$ of $M$ identical regions $m \in \mathbf{M}$, each with an identical autarkic equilibrium price $\bar{p}_{m}$, will satisfy:

$$
\sum_{m} D_{m}(\bar{p})=\sum_{m} S_{m}(\bar{p}) \Leftrightarrow M D_{m}(\bar{p})=M S_{m}(\bar{p}) \Longrightarrow \bar{p}=\bar{p}_{m}
$$

From:

$$
\varphi^{\prime}(\bar{p})=\frac{S^{\prime}(\bar{p})}{D^{\prime}(\bar{p})}=\sum_{m} \frac{D_{m}^{\prime}(\bar{p})}{D^{\prime}(\bar{p})} \varphi_{m}^{\prime}(\bar{p})
$$

and because $D^{\prime}(p)=\sum_{m: p \leq p_{0}^{m}} D_{m}^{\prime}(p)=M D_{m}^{\prime}(p) \mathbf{1}_{\left\{p \leq p_{0}^{m}\right\}}$ implies that $\frac{D_{m}^{\prime}(\bar{p})}{D^{\prime}(\bar{p})}=$ $\frac{1}{M}$ since $\bar{p} \leq p_{0}^{m}$, we have $\varphi^{\prime}(\bar{p})=\sum_{m} \frac{1}{M} \varphi_{m}^{\prime}(\bar{p})=\varphi_{m}^{\prime}(\bar{p})$. And by $\bar{p}=$ $\bar{p}_{m}, \varphi^{\prime}(\bar{p})=\bar{\varphi}_{m}^{\prime}\left(\bar{p}_{m}\right)$. Therefore,

$$
\left|\varphi^{\prime}(\bar{p})\right|<1 \Leftrightarrow\left|\varphi_{m}^{\prime}\left(\bar{p}_{m}\right)\right|<1 \Leftrightarrow S_{m}^{\prime}\left(\bar{p}_{m}\right)<\left|D_{m}^{\prime}\left(\bar{p}_{m}\right)\right|
$$

and proposition 3 in the text is extended to the class of non-linear agricultural economies; so that, conditional to an initial price restriction $p_{0} \in N_{\epsilon}(\bar{p}), \bar{p}$ is LSR if and only if $\bar{p}_{m}$ is also LSR. Q.E.D.

\section{Proof of Proposition 10}

Part (i): As only supply aggregates, $\alpha_{m}=\frac{D_{m}^{\prime}(\bar{p})}{D_{m}^{\prime}(\bar{p})}=1$ and $\left(\bar{p}_{m}-\bar{p}\right)>0$. Since aggregate demand is concave, this last fact implies that $\frac{D_{m}^{\prime}\left(\bar{p}_{m}\right)}{D_{m}^{\prime}(\bar{p})}>1$. As well, the concavity of supply implies that $\frac{S_{m}^{\prime}(\bar{p})}{S_{m}^{\prime}\left(\bar{p}_{m}\right)}>1$. Incorporating these observations in the above definition of the eductive stability condition of the integration equilibrium price, yields:

$$
\varphi^{\prime}(\bar{p})=\sum_{m} \alpha_{m} \frac{D_{m}^{\prime}\left(\bar{p}_{m}\right)}{D_{m}^{\prime}(\bar{p})} \frac{S_{m}^{\prime}(\bar{p})}{S_{m}^{\prime}\left(\bar{p}_{m}\right)} \varphi_{m}^{\prime}\left(\bar{p}_{m}\right) \leq \sum_{m} \varphi_{m}^{\prime}\left(\bar{p}_{m}\right)
$$

Because of the cobweb functions being (weakly) decreasing and $\alpha_{m} \frac{D_{m}^{\prime}\left(\bar{p}_{m}\right)}{D_{m}^{\prime}(\bar{p})} \frac{S_{m}^{\prime}(\bar{p})}{S_{m}^{\prime}\left(\bar{p}_{m}\right)}>$ 1. Taking absolute values on both sides of the inequality, and noticing that $\varphi_{m}^{\prime}()<0,. \forall m$ :

$$
\left|\varphi^{\prime}(\bar{p})\right| \geq\left|\sum_{m} \varphi_{m}^{\prime}\left(\bar{p}_{m}\right)\right|=\sum_{m}\left|\varphi_{m}^{\prime}\left(\bar{p}_{m}\right)\right| \geq\left|\varphi_{m}^{\prime}\left(\bar{p}_{m}\right)\right|
$$


So that the integration equilibrium price is more unstable than the original autarkic equilibrium.

Part (ii): As only demand aggregates, $\left(\bar{p}_{m}-\bar{p}\right)<0, \forall m$. Since both aggregate demand and supply are concave, this implies that $\frac{D_{m}^{\prime}\left(\bar{p}_{m}\right)}{D_{m}^{\prime}(\bar{p})}<1$ and $\frac{S_{m}^{\prime}(\bar{p})}{S_{m}^{\prime}\left(\bar{p}_{m}\right)}<1$. Incorporating these observations in the above definition of the eductive stability condition of the integration equilibrium price, yields:

$$
\varphi^{\prime}(\bar{p})=\alpha_{m} \frac{D_{m}^{\prime}\left(\bar{p}_{m}\right)}{D_{m}^{\prime}(\bar{p})} \frac{S_{m}^{\prime}(\bar{p})}{S_{m}^{\prime}\left(\bar{p}_{m}\right)} \varphi_{m}^{\prime}\left(\bar{p}_{m}\right) \geq \varphi_{m}^{\prime}\left(\bar{p}_{m}\right)
$$

Because $\varphi_{m}^{\prime}()<0,. \forall m$ and $\alpha_{m} \frac{D_{m}^{\prime}\left(\bar{p}_{m}\right)}{D_{m}^{\prime}(\bar{p})} \frac{S_{m}^{\prime}(\bar{p})}{S_{m}^{\prime}\left(\bar{p}_{m}\right)}<1$, taking absolute values on both sides of the inequality:

$$
\left|\varphi^{\prime}(\bar{p})\right| \leq\left|\varphi_{m}^{\prime}\left(\bar{p}_{m}\right)\right|
$$

The inequality above states that the resulting integration equilibrium price is more stable than the original autarkic equilibrium. Q.E.D.

\section{Proof of Proposition 11}

First, if there are no differences in the maximal willingnesses to pay across regions, $\sum_{m: \bar{p} \leq p_{0}^{m}} D_{m}^{\prime}(\bar{p})=\sum_{m} D_{m}^{\prime}(\bar{p})$ and $\varphi^{\prime}(\bar{p})=\sum_{m} \alpha_{m} \varphi_{m}^{\prime}(\bar{p})$. Although for non-linear economies $\varphi_{m}^{\prime}(\bar{p}) \neq \varphi_{m}^{\prime}\left(\bar{p}_{m}\right)$ since

$$
\varphi_{m}^{\prime}(\bar{p})=\frac{D_{m}^{\prime}\left(\bar{p}_{m}\right)}{D_{m}^{\prime}(\bar{p})} \frac{S_{m}^{\prime}(\bar{p})}{S_{m}^{\prime}\left(\bar{p}_{n}\right)} \varphi_{m}^{\prime}\left(\bar{p}_{m}\right)
$$

we can rather expand $\varphi_{m}^{\prime}($.$) as:$

$$
\varphi_{m}^{\prime}(\bar{p})=\varphi_{m}^{\prime}\left(\bar{p}_{m}\right)+\left(\bar{p}-\bar{p}_{m}\right) \int_{0}^{1} \varphi_{m}^{\prime \prime}\left[\bar{p}_{m}+\zeta\left(\bar{p}-\bar{p}_{m}\right)\right] d \zeta
$$

Which plugged into $\varphi^{\prime}(\bar{p})$ yields:

$$
\varphi^{\prime}(\bar{p})=\sum_{m} \alpha_{m} \varphi_{m}^{\prime}\left(\bar{p}_{m}\right)+\underbrace{\sum_{m} \alpha_{m}\left(\bar{p}-\bar{p}_{m}\right) \int_{0}^{1} \varphi_{m}^{\prime \prime}\left[\bar{p}_{m}+\zeta\left(\bar{p}-\bar{p}_{m}\right)\right] d \zeta}_{\equiv R \gtrless 0}
$$

Then $\varphi^{\prime}(\bar{p})-R=\sum_{m} \alpha_{m} \varphi_{m}^{\prime}\left(\bar{p}_{m}\right)$, which is a convex combination of the autarkic stability conditions. Therefore, taking absolute values on both sides:

$$
\min _{m}\left|\varphi_{m}^{\prime}\left(\bar{p}_{m}\right)\right| \leq\left|\varphi^{\prime}(\bar{p})-R\right| \leq \max _{m}\left|\varphi_{m}^{\prime}\left(\bar{p}_{m}\right)\right|
$$

Using the property that $\left|\varphi^{\prime}(\bar{p})-R\right| \geq|| \varphi^{\prime}(\bar{p})|-| R||$ and adding $+|R|$ to both sides of the second inequality in the above expression, we obtain:

$$
\left|\varphi^{\prime}(\bar{p})\right|=|| \varphi^{\prime}(\bar{p})|-| R|+| R|| \leq|| \varphi^{\prime}(\bar{p})|-| R||+|R| \leq \max _{m}\left|\varphi_{m}^{\prime}\left(\bar{p}_{m}\right)\right|+|R|
$$


Reaching the desired conclusion, for even if $\max _{m}\left|\varphi_{m}^{\prime}\left(\bar{p}_{m}\right)\right|<1$, so that all autarkic integrating economies are expectationally stable, the regional integration of them need not (even without differences in the maximal willingnesses to pay across regions). Finally, notice that for economies in the linear class $\mathbf{N}$ of proposition $6, R=0$ so that this proposition extends the results obtained there. But as well, notice that even in the non-linear case it can happen that $R=0$, as it is the case when the integrating economies are identical (proposition 9 above). Q.E.D.

\section{Proof of Proposition 12}

We have to prove that $\bar{p} \in\left[\min _{m} \bar{p}_{m}, \max _{m} \bar{p}_{m}\right]$. By contradiction, suppose that $\bar{p}>\max \bar{p}_{m}$. Call $\bar{m} \in \mathbf{M}$ the region the autarkic equilibrium of which is $\max _{m} \bar{p}_{m} \equiv \bar{p}_{\bar{m}}$. By (A.1.) and $\bar{p}>\bar{p}_{\bar{m}}, S_{\bar{m}}(\bar{p})>S_{\bar{m}}\left(\bar{p}_{\bar{m}}\right)=D_{\bar{m}}\left(\bar{p}_{\bar{m}}\right)>$ $D_{\bar{m}}(\bar{p})$. As $\bar{p}>\bar{p}_{\bar{m}} \equiv \max _{m} \bar{p}_{m}$ we have that $\bar{p}>\bar{p}_{m}, \forall m \neq \bar{m}$ and by (A.1.), $S_{m}(\bar{p})>S_{m}\left(\bar{p}_{m}\right)=D_{m}\left(\bar{p}_{m}\right)>D_{m}(\bar{p}), \forall m \neq \bar{m}$. Summing over all economies, $\sum_{m} S_{m}(\bar{p})=S(\bar{p})>D(\bar{p})=\sum_{m} D_{m}(\bar{p})$, a contradiction. Assuming that that $\bar{p}<\min _{m} \bar{p}_{m}$ and denoting by $\underline{m} \in \mathbf{M}$ the region whose autarkic equilibrium is $\min _{m} \bar{p}_{m}$, by reversing the inequalities in the preceding reasoning we similarly reach a contradiction. Q.E.D. 